\title{
EVALUATING THE EFFECTIVENESS OF CRANIAL MOLDING FOR TREATMENT OF POSITIONAL PLAGIOCEPHALY USING FINITE ELEMENT ANALYSIS
}

\author{
A Thesis \\ presented to \\ The Faculty of California Polytechnic State University, \\ San Luis Obispo
}

\author{
In Partial Fulfillment \\ of the Requirements for the Degree \\ Master of Science in Engineering with a \\ Specialization in Biomedical Engineering
}

by

Maziyar Keshtgar

May 2015 
(C) 2015

Maziyar Keshtgar

ALL RIGHTS RESERVED 
TITLE:

AUTHOR:

DATE SUBMITTED:

COMMITTEE CHAIR:

COMMITTEE MEMBER:

COMMITTEE MEMBER:
Evaluating the Effectiveness of Cranial Molding for Treatment of Positional Plagiocephaly Using Finite Element Analysis

Maziyar Keshtgar

May 2015

Scott Hazelwood, Ph.D.

Professor of Biomedical Engineering

David Clague, Ph.D.

Professor of Biomedical Engineering

Daniel W. Walsh, Ph.D.

Professor of Biomedical Engineering 


\section{ABSTRACT \\ Evaluating the Effectiveness of Cranial Molding for Treatment of Positional Plagiocephaly Using Finite Element Analysis \\ Maziyar Keshtgar}

Since the advent of recommendations for placing infants in the supine position during sleep to reduce the incidence of sudden infant death syndrome, clinicians have noted an increase in the frequency of cranial asymmetry due to deformation of suture sections of the infants' skulls as a result of constant concentrated stress in one area at the back of their head. This specific form of cranial deformation is known as positional plagiocephaly and its rate of occurrence has increased from $0.3 \%$ in $8.2 \%$ within the past 30 years.

Current treatments and methodologies for preventing and correcting positional plagiocephaly such as stretching exercises, bedding pillows, and cranial molding are not optimized for effectiveness and comfort. Literature surrounding the implementation of these methodologies or devices often assesses the relative effectiveness of each treatment through statistical means, or studies complications associated with their use. There is a lack of quantified mechanical analysis for determining the effectiveness of each treatment or engineered solutions.

In this study, a finite element model was created and validated to study the effect of wearing a cranial helmet, as the most effective non-surgical device for treatment of positional plagiocephaly, on reducing concentrated stress from the back of the baby's head during sleep. The results from this model were then compared to two other finite element models with a healthy baby sleeping in supine position on a pillow, and a patient diagnosed with a severe case of positional plagiocephaly sleeping on the flat side of his head in supine position. The geometries representing the head of the babies in these models are the refined 3D laser- 
scanned file of a patient's head contour at Hanger Clinic as well as the cavity inside the cranial helmet that was used for treatment of the baby.

After successfully assigning section and contact properties to different regions of the models, applying proper loading and boundary conditions, and performing mesh convergence studies for each of the three models, the average Von Mises stress values of each of the 13 different suture segments of each model were summarized in tables and evaluated using mathematical and qualitative methods.

The stress value data obtained from different suture regions of the model with the cranial helmet resulted in the smallest standard deviation among all three populations which supports that wearing the cranial helmet helps to reduce stress concentrations. Use of the cranial helmet during sleep also showed a significant decrease of the average Von Mises stress within the posterior fontanelle by $90 \%$ compared to the healthy baby sleeping in supine position and $73.4 \%$ compared to the deformed head sleeping on the flat surface of the head.

The major limitations of this study are correlated with the simplifying assumptions and geometries in generating and validating the models. Future studies need to focus on overcoming these limitations and generating more complex models using a similar approach. The methods used in this study and the results obtained from the models can serve as a basis for future development of engineered solutions that are more effective than the existing solutions in the market and reduce the side-effects and complications associated with their use.

Keywords: Cranial Molding, Cranial Helmet Therapy, Positional Plagiocephaly, Deformational Plagiocephaly, Cranial Asymmetry, Corrective Orthotic Device, Finite Element Analysis, Von Mises Stress 


\section{ACKNOWLEDGMENTS}

l'd like to personally thank Dr. Scott Hazelwood, for giving me the opportunity to have him as my thesis advisor and use his guidance in this research. I'd also like to thank Dr. Mathew Robinson and Hanger Clinic for making the data used in this study accessible. Lastly, l'd like to thank my family for the great help and support throughout my education. 


\section{TABLE OF CONTENTS}

Page

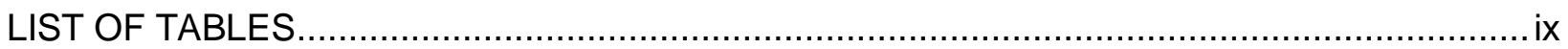

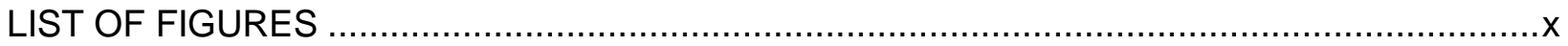

\section{CHAPTER}

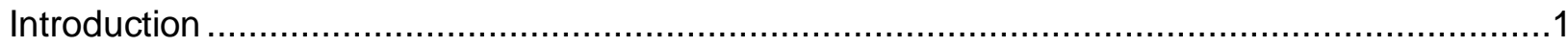

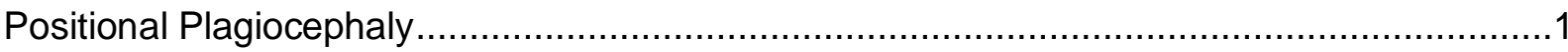

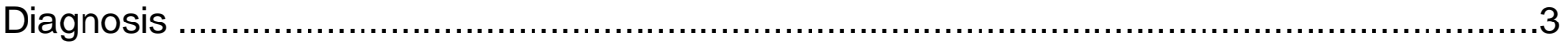

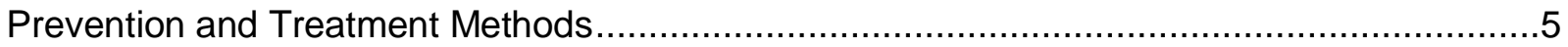

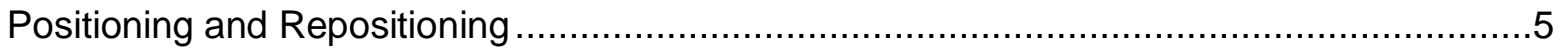

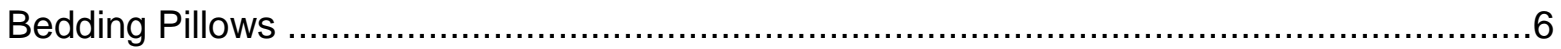

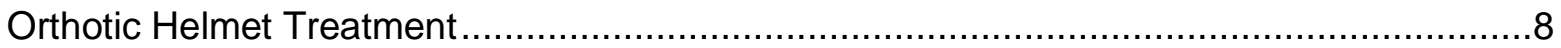

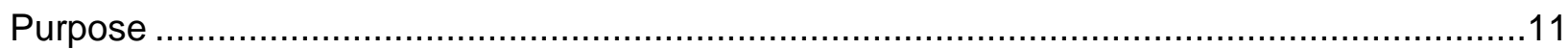

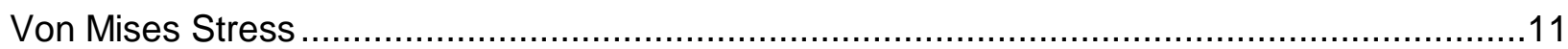

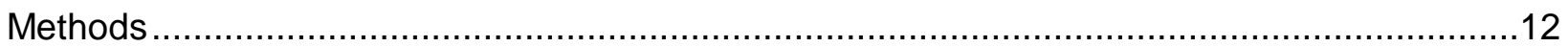

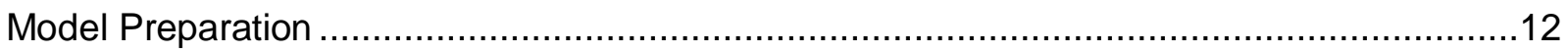

Model 1: A Healthy Baby Sleeping on a Pillow in Supine Position ....................................19

Model 2: The PP Patient Sleeping on a Pillow in Supine Position .....................................19

Model 3: The PP Subject Sleeping in Supine Position Wearing the Cranial Helmet..............20

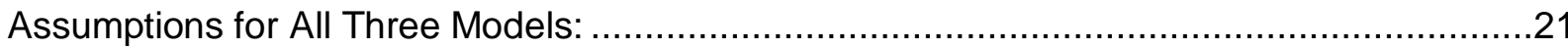

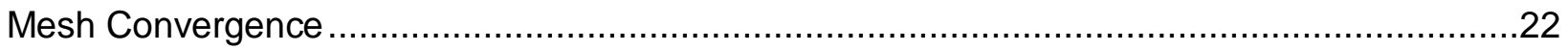

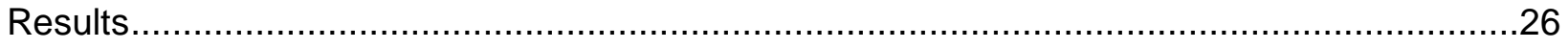

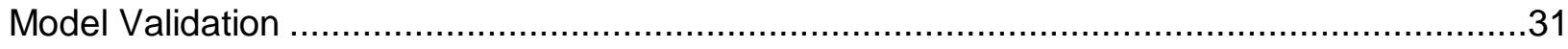

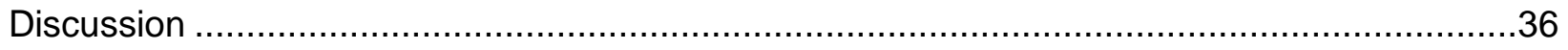

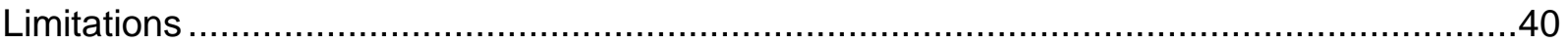

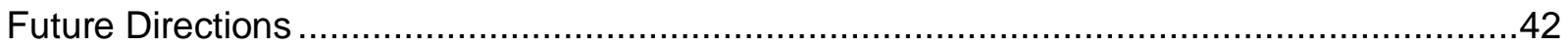




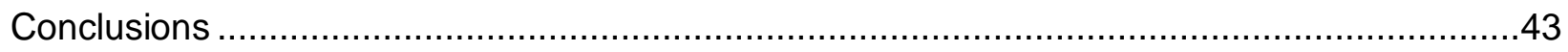

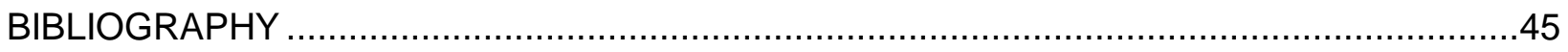

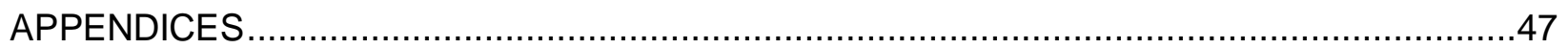

Appendix A: Color Contour Plots of Von Mises Stress Distribution within Suture Segments of

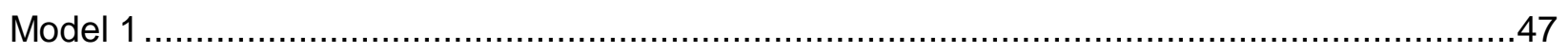

Appendix B: Color Contour Plots of Von Mises Stress Distribution within Suture Segments of

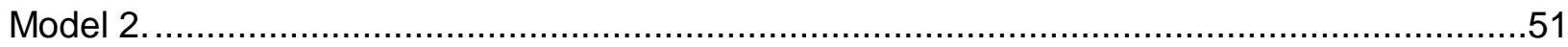

Appendix C: Color Contour Plots of Von Mises Stress Distribution Within Suture Segments of

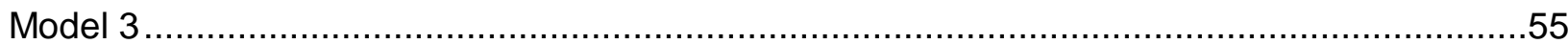




\section{LIST OF TABLES}

Table

Page

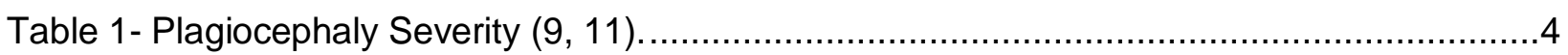

Table 2- Material properties used for the helmet, bones, sutures, and the pillow....................22

Table 3- Stress Value Results for Model 1: Number of elements, minimum, maximum, and average Von Mises stress values off all 13 different suture sections for the baby with a healthy skull sleeping in supine position on a pillow.

Table 4- Stress Value Results for Model 2: Number of elements, minimum, maximum, and average Von Mises stress values off all 13 different suture sections for PP patient sleeping in supine position on the flat side of his head on a pillow.

Table 5- Stress Value Results for Model 3: Number of elements, minimum, maximum, and average Von Mises stress values off all 13 different suture sections for the PP patient in supine position wearing an orthotic helmet.

Table 6- Descriptive Satistics of the three populations of average Von Mises stress values......30

Table 7- Comparing the calculated pressure values.

Table 8- Growth related parameters and morphometric measurements used in the study

performed by Schweitzer e al (32).

Table 9- The results of the morphometric analysis at T1 and T2 (32).

Table 10- Comparing average Von Mises Stress Values of Left Lamboidal, Right Lamboidal, Sagittal, and Posterior sutures obtained from models 1 and 3. 


\section{LIST OF FIGURES}

Figure $\quad$ Page

Figure 1- Normal Skull vs. Deformational Plagiocephaly (1) ..........................................

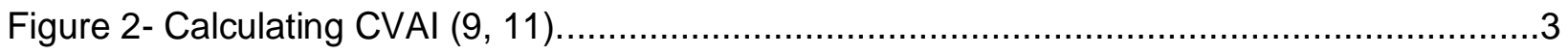

Figure 3- Photos and 3D Computer Tomography (CT) Images of a PP Patient: Pre- and Post-

Treatment. The treatment initiation age was 6.3 months and the treatment period was 7.1

months .A) The initial CVAI was $11.46 \%$. B) The CVAl decreased by $8.19 \%$ at the end of the treatment period. C) 3D CT scan before the therapy was performed to confirm that craniosynostosis was not present. Any discrepancy in the diagonal distances was also noted in the 3D scan. D) The 3D CT scan was performed again after the treatment to assess the treatment effect. The results showed the decreases discrepancy of the CVAI (13).......

Figure 4- Stretching exercises: A) Stretching the cervical musculature in right/left rotatuin, and hold 10 seconds. B) Stretching the cervical musculature in right/left flexion, hold 10 seconds (12) 6

Figure 5- A) A bedding pillow designed to prevent PP. B) Use of the bedding pillow (12)..........7

Figure 6- Orthotic helmet and its use (1).... 8

Figure 7- Plaster Casting Process- A: Head is covered in cotton stocking to protect the baby's skin. B: Ear, eyebrows, and centerline are marked. C: A piece of plaster is placed behind the head. D: A second interlocking piece is fit over the top. E: Plaster pieces harden in a couple of minutes and then immediately removed and put back together to generate an exact representation of the skull. F: The molded representation of the skull would then be used for manufacturing the cranial helmet (20).

Figure 8- Use of 3D laser scanning technology: A) Creating a 3D computer image of the patient's head geometry B) Using CAD software to record and modify the 3D files. C) Custom cranial helmet created using the CAD files and CAM technologies (21).

Figure 9- Skin irritation and discomfort caused by wearing the orthotic helmet (24). 10 
Figure 10- Effect of the cranial molding treatment on correcting the patient's head asymmetry: Compares before (March 2012) and after (June 2012) treatment head contours. ..13

Figure 11- Insignia's 3D laser scanner technology at Hanger Clinic (25). 13

Figure 12- The original STL file of the patient's scanned head surface contour: Contains

28000 triangles.

Figure 13- The modified water-tight STL file of the patient's scanned head surface contour

with 6000 triangles. 15

Figure 14- Tracing cranial sutures over the modified 3D computer image according to the

CT scanned images (5). 16

Figure 15- Creating the 3D model of the orthotic helmet: Reconstructed based on the helmet cavity file provided by Hanger Clinic.

Figure 16- Solid model of the pillow used to create the FE models. .....................................18

Figure 17- The loading, boundary, and surface contact conditions for model 1 ....................19

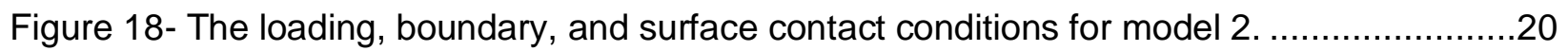

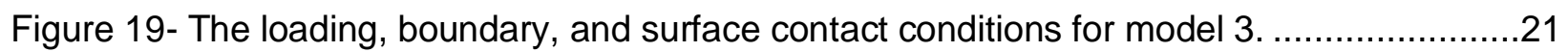

Figure 20- Mesh convergence plot of Model 1: The convergence values shown in the table.

The red arrow points to the node used for generating the plot.........................................23

Figure 21-Mesh convergence plot of Model 2: The convergence values shown in the table.

The red arrow points to the node used for generating the plot.

Figure 22- Mesh convergence plot of Model 3: The convergence values shown in the table.

The red arrow points to the node used for generating the plot. .25

Figure 23- Color contour plot of Von Mises stress distribution for model 1: baby with a healthy skull sleeping in supine position on a pillow. .26

Figure 24- Color contour plot of Von Mises stress distribution for model 2: PP patient sleeping in supine position on the flat side of his head on a pillow. 
Figure 25- Color contour plot of Von Mises stress distribution for model 3: PP patient

sleeping in supine position wearing an orthotic helmet.

Figure 26- Interval plots of the three populations of average Von Mises values for models

1, 2 and 3.

Figure 27- Side-by-side comparison of average Von Mises stress values for all 13 individual

suture regions of models 1,2 , and 3 .

Figure 28- Color contour plot of pressure distribution over the surface of the pillow in Model 1.

Pressure values are in PSI.

Figure 29- Color contour plot of pressure distribution over the surface of the pillow in Model 2.

Pressure values are in PSI.

Figure 30- Color contour plot of pressure distribution over interior surface of the helmet in Model 3. Pressure values are in PSI.

Figure 31- Orientation of three dimensional measurements. Setting up x-axis, y-axis, z-axis, and 0-plane: Measurement plane is the 0-plane shifted parallel to the level of maximum posterior curvature in the occiput (left). Demonstration of volume quadrants from 0-plane (right) (31). 
Introduction

Positional Plagiocephaly

Since the advent of recommendations for placing infants in the supine position during sleep to reduce the incidence of sudden infant death syndrome, clinicians have noted an increase in the frequency of cranial asymmetry (1). This cranial asymmetry is also known as plagiocephaly. Cranial asymmetry in the absence of synostosis of the sutures is described as nonsynoptic posterior plagiocephaly. Positional plagiocephaly (PP) is always of this type and is also referred to as deformational plagiocephaly because of the effects of forces to deform the skull shape in the supine position (1). Unilateral flattening of the occiput with ipsilateral anterior shifting of the ear (Figure 1) are common signs of PP (1).
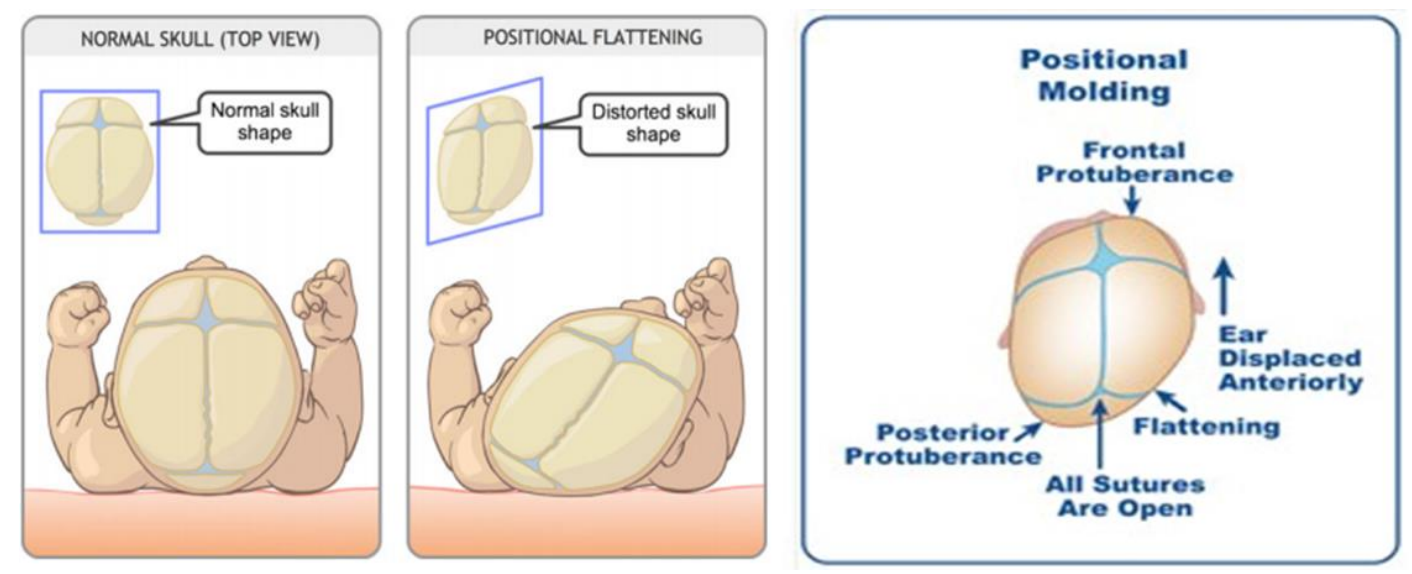

Figure 1- Normal Skull vs. Deformational Plagiocephaly (1).

In 1974, plagiocephaly occurred once every 300 live births among prone-sleeping infants.

(2) After the initiation of the "Back to Sleep" campaign, the frequency of plagiocephaly increased to 1 in 60 in 1996 (3). The incidence of this deformity is estimated to have increased from $0.3 \%$ to $8.2 \%$ or even higher today, depending on the sensitivity of the criteria used for diagnosing it (4). 
PP occurs at six weeks of age, increases to a maximum at four months, and then slowly decreases over two years because most cases resolve in that time. A large longitudinal study of 7,609 Dutch infants, published in 2010 , showed a persistence of occipital asymmetry in about $5 \%$ of all children by the third year of life (5).

Factors increasing the risk of PP are male sex, firstborn, limited passive neck rotation at birth (congenital torticollis), multiple births, breech births, low birth weight, supine sleeping position at birth and at six weeks, bottle feeding, breast feeding fewer than three times per day, and lower activity level with slower achievement of milestones $(6,7)$. Sleeping with the head to the same side and positional preference when sleeping are also associated with the development of PP (6). The side of occipital flattening correlates strongly to the side that the head faces when in the supine sleep position (8).

It has been determined that brain volume quadruples and brain size increases to $75 \%$ of its adult volume during the first two years after birth (7). Also, about $85 \%$ of cranial growth takes place during the first year of postnatal life. (9) Therefore, treatment strategies focus primarily on this age group (9). PP must be differentiated from a pre-mature unilateral coronal or lamboidal synostosis (posterior synostoses), both of which will require surgical vault correction of the fused suture (7).

Long-term effects of positional plagiocephaly on development of a baby remain undefined (10). A study performed by M. F. Shamji et. al. is one of the very few publications in the literature that tried to define the cosmetic and cognitive outcomes observed following management of positional plagiocephaly (10). The study evaluated if the side of plagiocephaly could impact on the child's neurocognitive development and outcome. Surveys collected from parents of patients treated for positional plagiocephaly through the Children's Hospital of Eastern Ontario interrogating costmetic outcome, school performance, language skills, cognitive development and societal fuction were used to test the outcomes dependent on gender, age, and plagiocephaly side at the 0.05 level of confidence (10). The results suggested that non- 
surgical plagiocephaly management achieved good cosmetic outcome among patients in the study. Children with deformities on the left side of their head frequently encountered difficulties with cognitive and scholastic activities; however, the roles of the underlying disease and the treatment measures in this delay cannot be differentiated (10).

\section{Diagnosis}

The most commonly used technique by clinicians for diagnosing PP and quantifying the severity of this disease is calculating the Cranial Vault Asymmetry Index (CVAI). The longest and shortest diagonal from the forehead to the posterior skull are measured (Figure 2) and used to calculate the CVAI using the following formula (9):

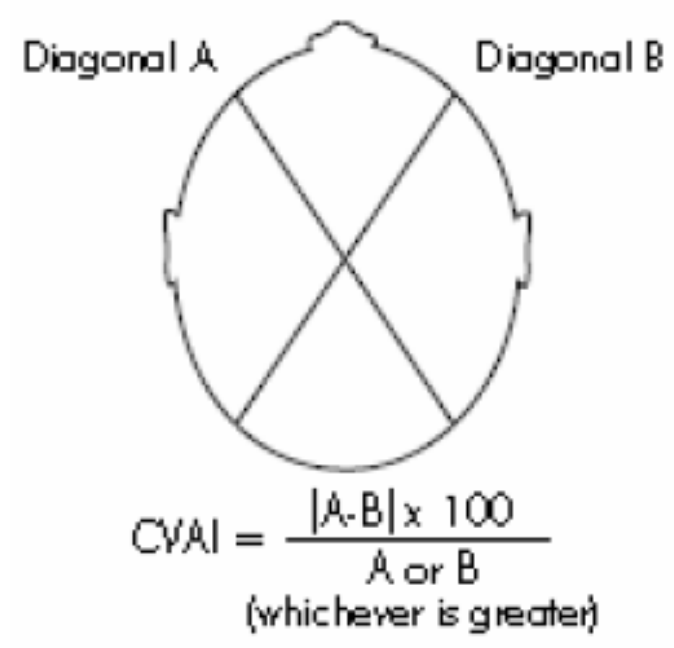

Figure 2- Calculating $\operatorname{CVAI}(9,11)$.

Measurements must be made in millimeters $(\mathrm{mm})$ at $30^{\circ}$ from center of nose (outer edge of eyebrow).The output of the equation would then correspond to one of the following five severity classifications (9): 


\begin{tabular}{|c|c|c|c|}
\hline Level & Clinical Presentation & Recommendation & CVAI \\
\hline $\begin{array}{c}1 \\
\text { Normal }\end{array}$ & All symmetry with in normal limits & No treatment & $<3.5 \%$ \\
\hline $\begin{array}{c}2 \\
\text { Mild }\end{array}$ & $\begin{array}{l}\text { - Minimal asymmetry in one } \\
\text { posterior quadrant } \\
\text { - No secondary changes }\end{array}$ & $\begin{array}{l}\text { - Repositioning program } \\
\text { - If } 2 \text { Month of repositioning } \\
\text { has failed, cranial } \\
\text { remolding orthosis at } \\
\text { parent's request for } \\
\text { cosmetic purposes }\end{array}$ & $3.5 \%$ to $6.25 \%$ \\
\hline $\begin{array}{c}3 \\
\text { Moderate }\end{array}$ & $\begin{array}{l}\text { - Two quadrant involvement } \\
\text { - Moderate to severe posterior } \\
\text { quadrant flattening } \\
\text { Minimal ear shift and/or anterior } \\
\text { involvement }\end{array}$ & $\begin{array}{l}\text { - Repositioning program or } \\
\text { - Cranial remolding orthosis- } \\
\text { based on age and history }\end{array}$ & $6.25 \%$ to $8.75 \%$ \\
\hline $\begin{array}{c}4 \\
\text { Severe }\end{array}$ & $\begin{array}{l}\text { - } \quad \text { Two or three quadrant involvement } \\
\text { - Severe posterior quadrant flattening } \\
\text { - } \quad \text { Moderate ear shift } \\
\text { - Anterior involvement including } \\
\text { noticeable orbit asymmetry }\end{array}$ & Cranial remolding orthosis & $8.75 \%$ to $11.0 \%$ \\
\hline $\begin{array}{c}5 \\
\text { Very Severe }\end{array}$ & $\begin{array}{l}\text { - } \quad \text { Three or four quadrant involvement } \\
\text { - Severe posterior quadrant flattening } \\
\text { - Severe ear shift } \\
\text { - Anterior involvement including } \\
\text { orbit and cheek asymmetry }\end{array}$ & Cranial remolding orthosis & $>11.0 \%$ \\
\hline
\end{tabular}

Table 1- Plagiocephaly Severity $(9,11)$.

Using the severity scale (Table 1), the clinician would then decide which type of treatment would be best for the patient. For severe cases, cranial helmet treatment is usually implemented (12). Figure 3 demonstrates an example of how measurement of CVAI is implemented in clinical applications. 

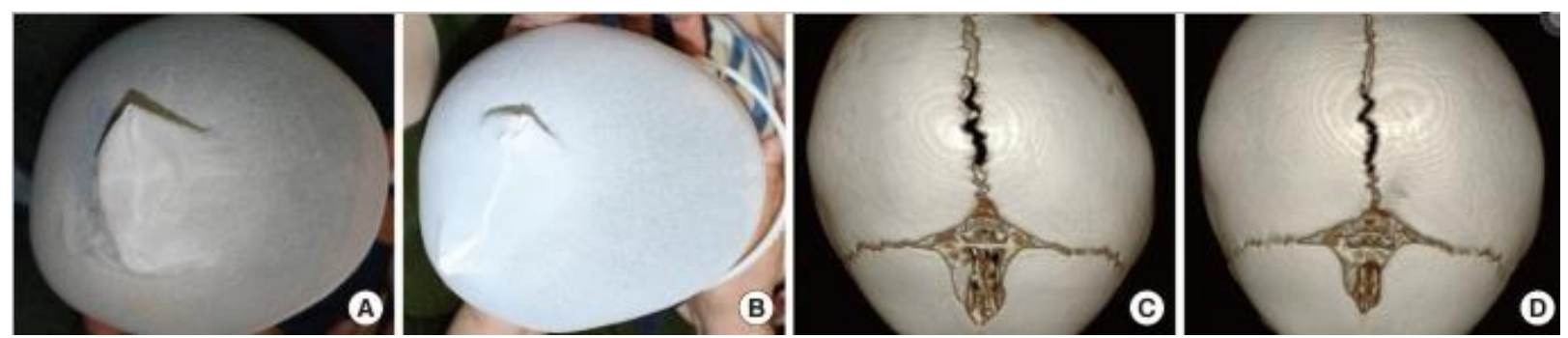

Figure 3- Photos and 3D Computer Tomography (CT) Images of a PP Patient: Pre- and PostTreatment. The treatment initiation age was 6.3 months and the treatment period was 7.1 months .A) The initial CVAl was $11.46 \%$. B) The CVAl decreased by $8.19 \%$ at the end of the treatment period. C) 3D CT scan before the therapy was performed to confirm that craniosynostosis was not present. Any discrepancy in the diagonal distances was also noted in the 3D scan. D) The 3D CT scan was performed again after the treatment to assess the treatment effect. The results showed the decreases discrepancy of the CVAI (13).

Other examination methods for diagnosis of PP involve measuring the head circumference, palpation of the anterior fontanel and each cranial suture for ridging, evaluating the range of motion of the neck (chin-to-chest, chin-to shoulder, and ear-to-shoulder flexibility), and examination of the facial symmetry including the mandible, ears, eyes, and forehead (7). Prevention and Treatment Methods

Positioning and Repositioning

Primary prevention should be based on perinatal parental education on how and how often to change the infant's head position during sleep to avoid the incidence of cranial deformity $(1,14)$.

A study longitudinally evaluated 161 children with PP who were treated with "repositioning" only. By preschool, only $61 \%$ of children achieved normal skull contour, and $4 \%$ still had severe residual deformities (7).

Secondary prevention should be through the use of physiotherapy, osteopathic medicine, positioning devices, and engineered products such as bedding pillows. A study performed on 1086 Hong Kong infants with occipital flattening showed that $91 \%$ of the infants had resolved craniofacial asymmetry after physiotherapy (manual stretching) (14) . 
Repositioning is inexpensive compared to the other treatments; however, it requires dedicated parents who are willing and are able to proactively and continuously alter their child's head position and room logistics (13). It also requires at least 60 minutes of awake active "tummy time" and/or stretching exercises similar to the method shown in figure 4.
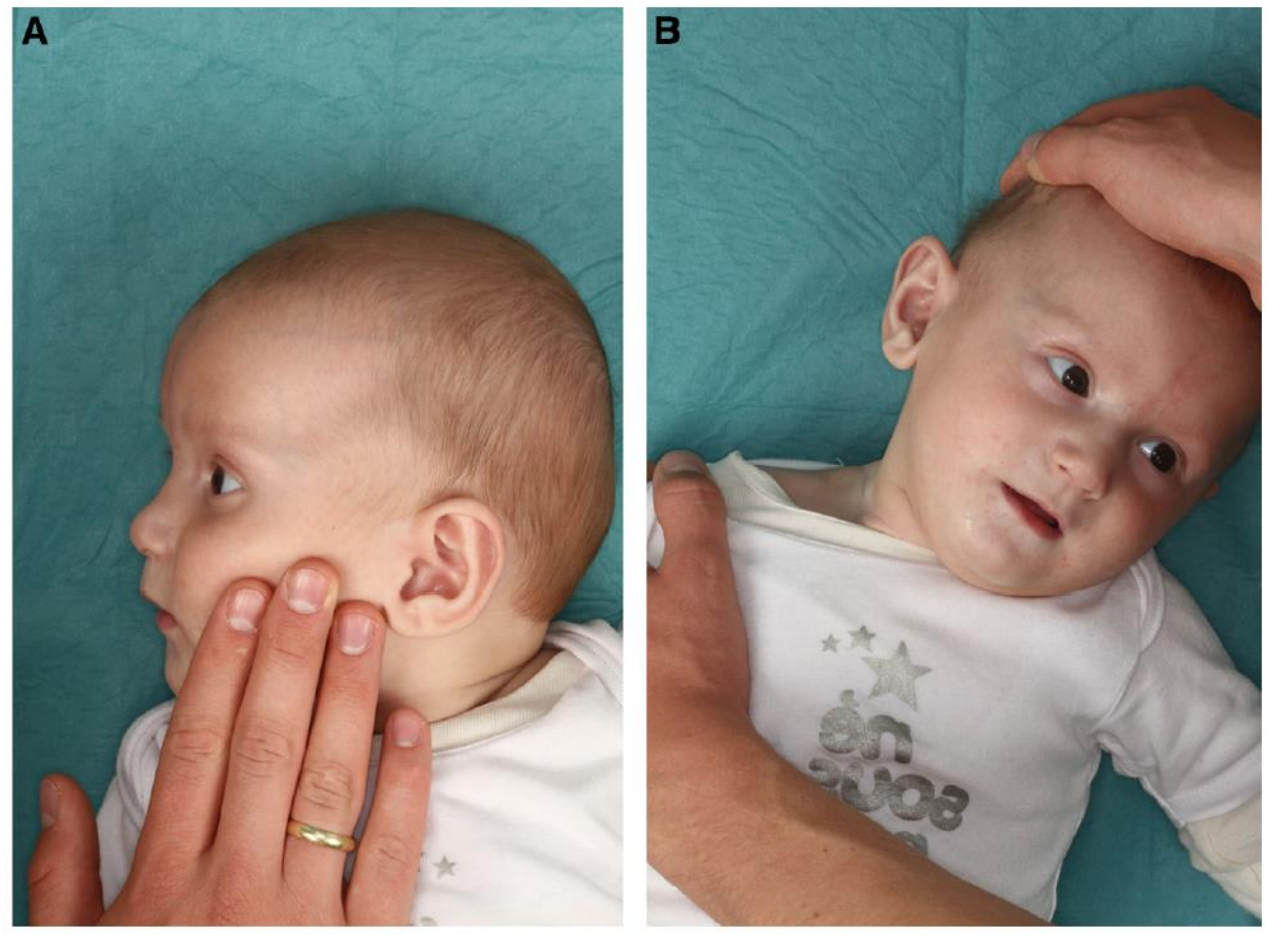

Figure 4- Stretching exercises: A) Stretching the cervical musculature in right/left rotatuin, and hold 10 seconds. B) Stretching the cervical musculature in rightlleft flexion, hold 10 seconds (12).

This method may arguably be more acceptable for females since they can rely on customarily longer hair as they grow to cover their deformity if the PP does not improve significantly (13).

\section{Bedding Pillows}

Bedding pillows (Figure 5) are designed to relieve the cranial pressure from the back of the baby's head while sleeping in supine position $(16,17)$. Although parents often find using these pillows advantageous during early therapies and report improvements in head 
deformities, the efficacy of this method as a treatment for fixing severe deformations has not yet been systematically analyzed or proven $(17,18)$. Therefore, these products are considered as preventative devices or early treatments only. All of these preventative methods should be started as soon as the child is born and be continued in an adequate manner to achieve improvements (16). However, if no satisfactory improvement of the cranial shape is achieved, orthotic helmet treatment should be initiated at an adequate age for the effective correction of the cranial deformities $(16,18)$.
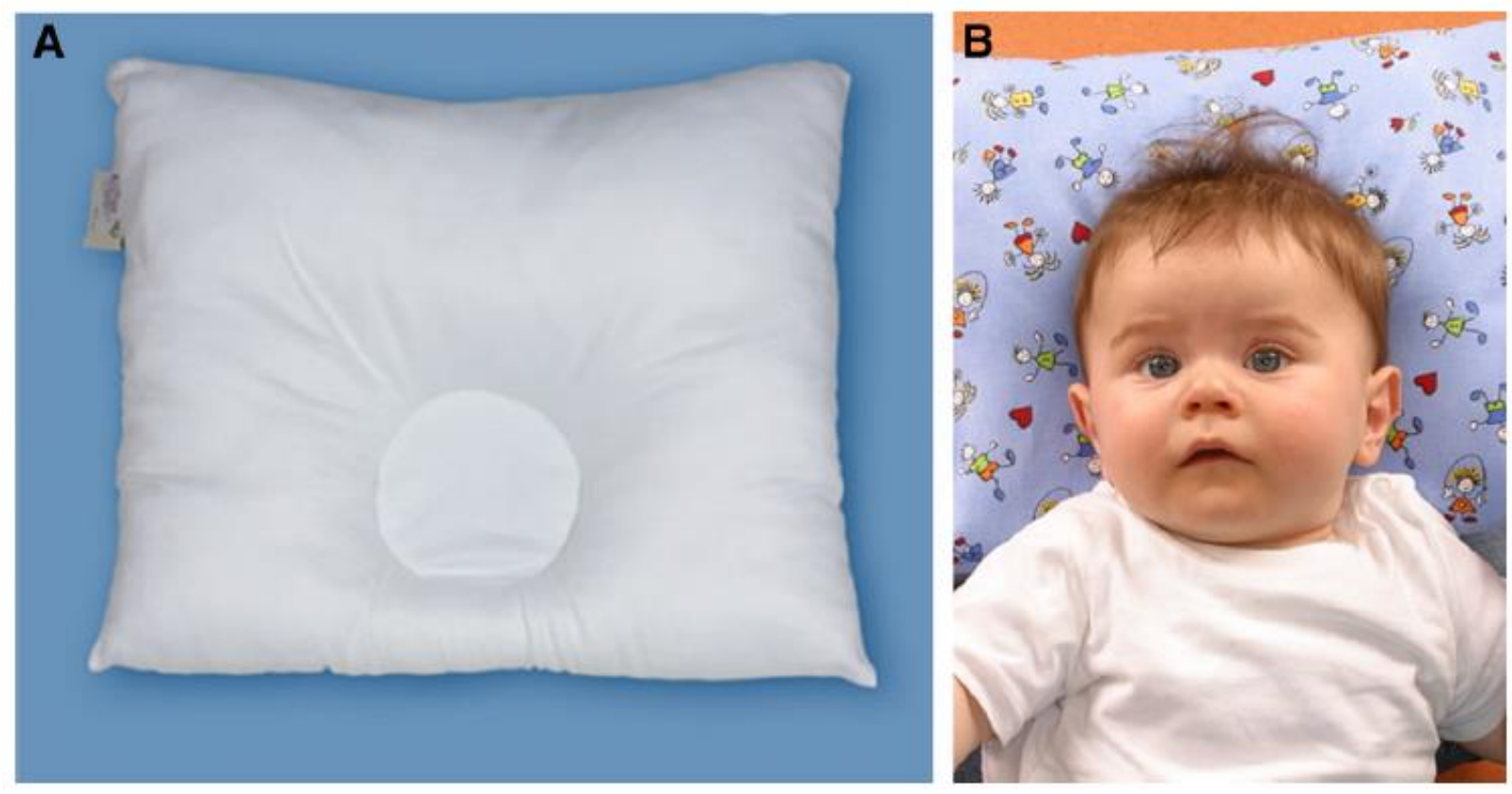

Figure 5- A) A bedding pillow designed to prevent PP. B) Use of the bedding pillow (12).

A group of 50 children aged 5 months or younger diagnosed with PP were included in a prospective clinical trial to evaluate the impact of stretching exercises versus available bedding pillows on positional plagiocephaly. The results suggested that stretching exercises and bedding pillows resulted in improvements in positional head deformities while improvements were slightly greater when using bedding pillows versus stretching (16). 


\section{Orthotic Helmet Treatment}

Orthotic helmet treatment (Figure 6) involves creating a cranial molding helmet around the child's head so that the baby's skull fills the void areas within the helmet as the baby's head grows rapidly in the early stages of his/her life. Orthotic helmet treatment has been known to result in a significantly better result when treating severe cases of PP (18). Most favorable cosmetic outcomes can be achieved when this treatment is applied early on infants between 612 months of age (19).

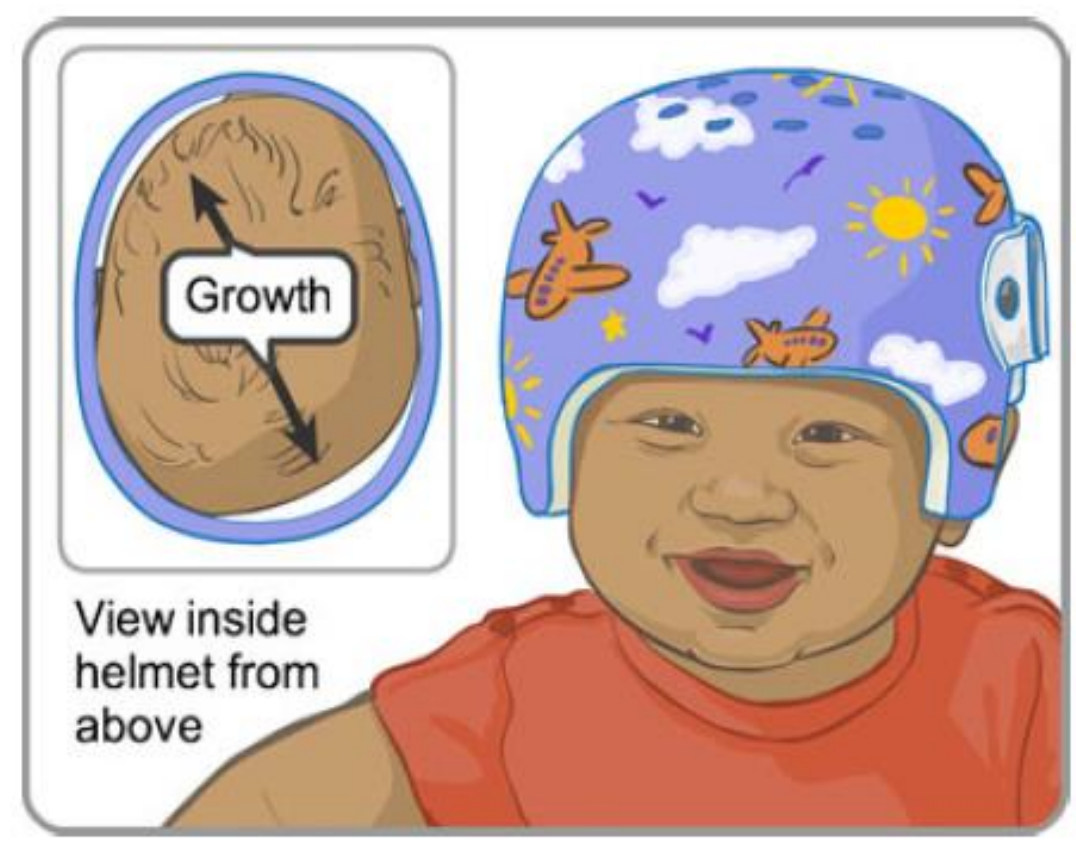

Figure 6- Orthotic helmet and its use (1).

The process involves measuring the baby's head to keep track of the progress during each successive visit. Some clinics create a plaster cast of the child's head for use in making of the helmet and photographically record the head shape of the baby during each visit (Figure 7). However, more modern techniques nowadays involve using a 3D laser scanner (Figure 8) to create a 3D computer model of the baby's head geometry which can be used for recording the baby's progress during each visit as well as designing, manufacturing, and modifying the helmet using CAD software and CAM equipment. 


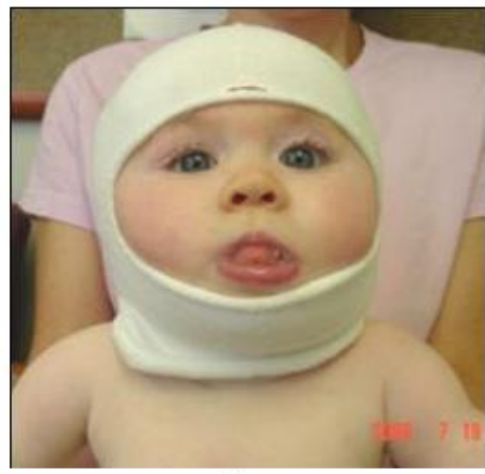

A

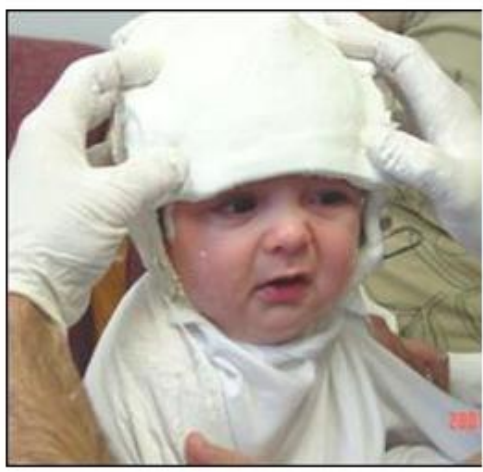

D

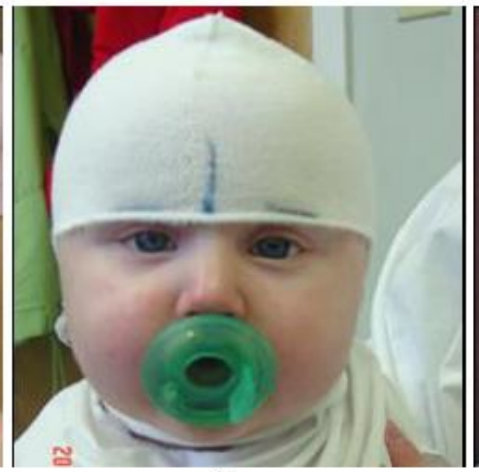

B

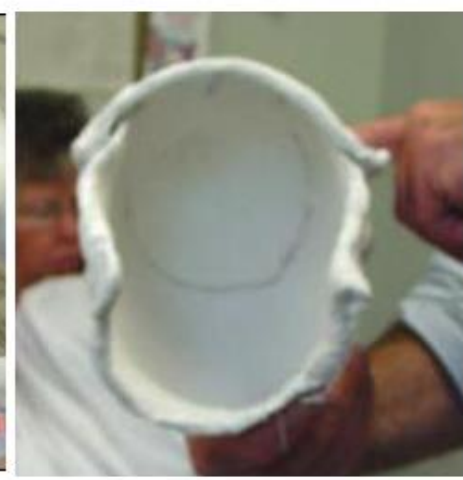

E

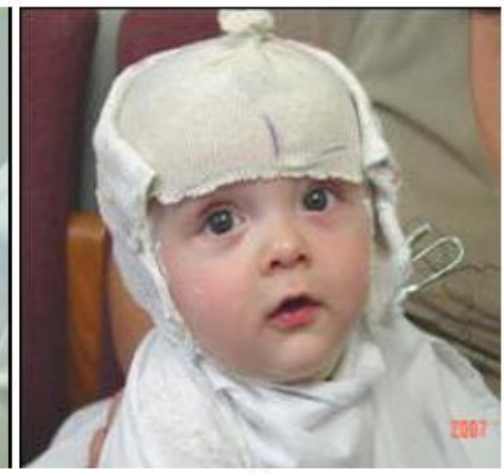

C

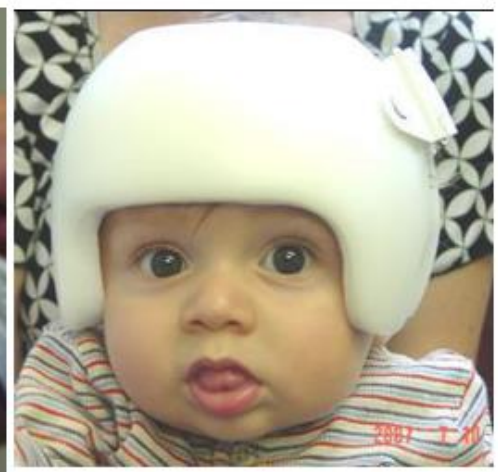

$\mathrm{F}$

Figure 7- Plaster Casting Process- A: Head is covered in cotton stocking to protect the baby's skin. B: Ear, eyebrows, and centerline are marked. C: A piece of plaster is placed behind the head. D: A second interlocking piece is fit over the top. E: Plaster pieces harden in a couple of minutes and then immediately removed and put back together to generate an exact representation of the skull. F: The molded representation of the skull would then be used for manufacturing the cranial helmet (20).

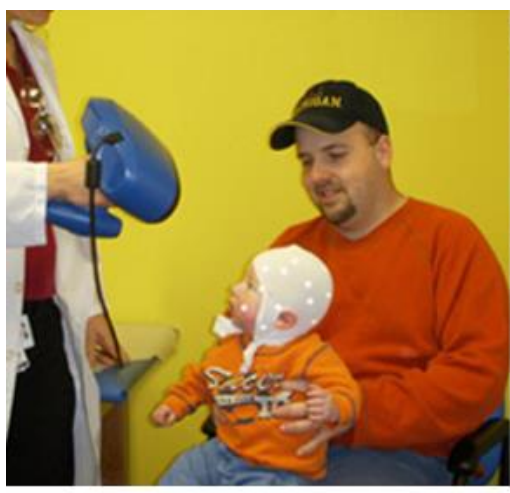

A

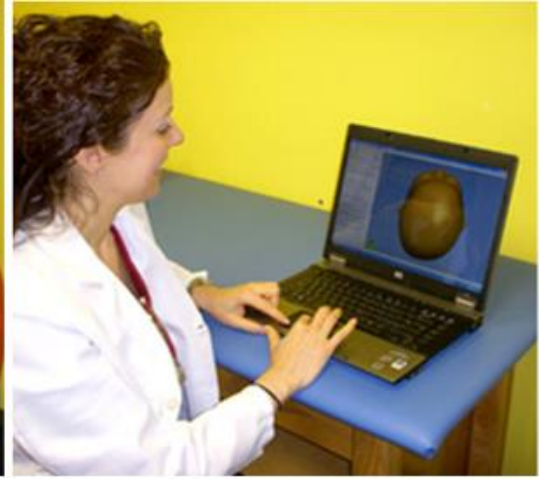

B

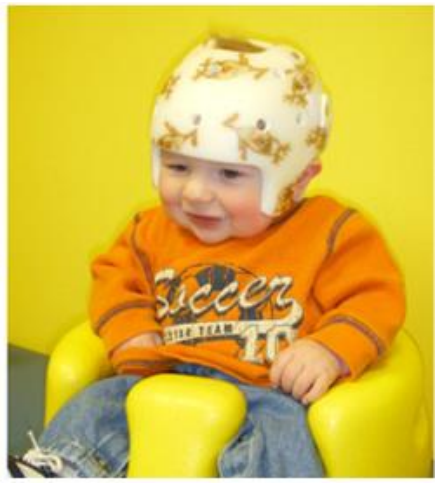

C

Figure 8- Use of 3D laser scanning technology: A) Creating a 3D computer image of the patient's head geometry B) Using CAD software to record and modify the 3D files. C) Custom cranial helmet created using the CAD files and CAM technologies (21). 
A study performed by Se Yon Kim, et al. compared "positioning" with "orthotic helmet therapy" in 298 consecutive infants referred for correction of head symmetry. The results of the study revealed that helmet therapy is efficacious in terms of correcting cranial asymmetry and ear shift, while counter positioning therapy is only effective in correcting the patient's ear shift (22). In addition, patients with moderate to severe deformational plagiocephaly showed superior improvements in terms of correcting cranial asymmetry and ear shift compared to the patients receiving only active repositioning treatments (22).

Cranial molding and orthotic helmet treatment is expensive if compared to other treatment methods, averaging about $\$ 3000$ for just the orthoses, plus requiring multiple office visits to both the orthotic supplier and the subspecialists (7). In addition, commercial insurance companies in the US rarely cover the costs of the helmet orthoses, except for post-synostoses surgery (7). Orthotic helmet treatment may also cause scalp and skin rashes (Figure 9), including seborrhea capitis, while it may make parents self-conscious about their child's problem $(7,23)$. Another inconvenience of this method is the discomfort resulted from wearing the orthoses for about 23 hours every day for about 2 to 4 months at minimum (7).

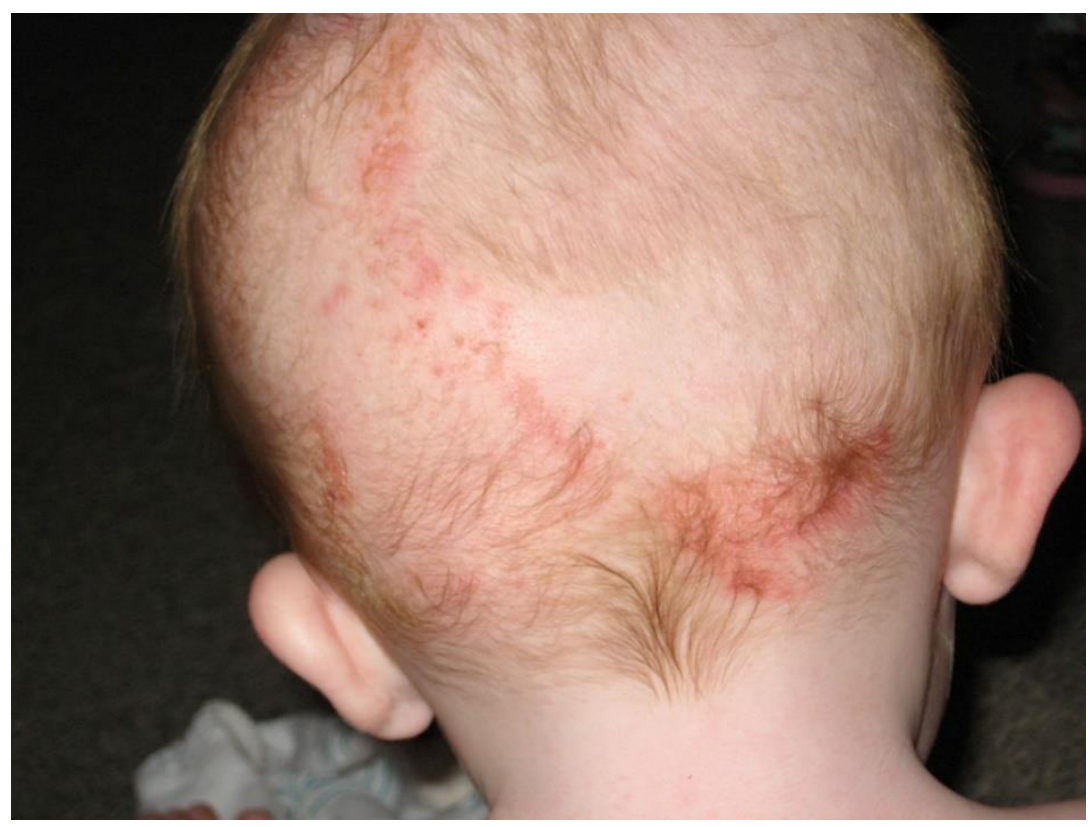

Figure 9- Skin irritation and discomfort caused by wearing the orthotic helmet (24). 


\section{Purpose}

The literature surrounding PP lack a focus on quantifying and analyzing the stress distribution throughout an infant's skull while sleeping in different positions or when wearing a corrective cranial helmet or using bedding pillows. The goal of this study is to develop and validate three separate Finite Element (FE) models of a baby with a healthy skull sleeping in supine position, a baby diagnosed with PP sleeping in supine position with the flat side of the head directly contacting the pillow, and a baby diagnosed with PP sleeping in supine position wearing a cranial helmet to quantify and analyze the difference between the resulted Von Mises Stress distribution due to similar loading conditions and different boundary conditions. The focus will mainly be on the suture segments of the skulls with the FE models since deformation mostly occurs as a result of stress on the soft sutures in charge of skull growth. The results from this study may help to optimize the design, comfort, and effectiveness of engineering solutions for correcting and preventing PP.

\section{Von Mises Stress}

Von Mises stress will be used to quantify the stress distribution within the baby's skull and find areas of high and low stress for all three cases. Von Mises Stress is an equivalent or effective stress at which yielding is often predicted to occur in ductile materials under complex loading conditions.

$$
\left[\frac{\left(\sigma_{1}-\sigma_{2}\right)^{2}+\left(\sigma_{2}-\sigma_{3}\right)^{2}+\left(\sigma_{3}-\sigma_{1}\right)^{2}}{2}\right]^{1 / 2}=\sigma_{v}
$$

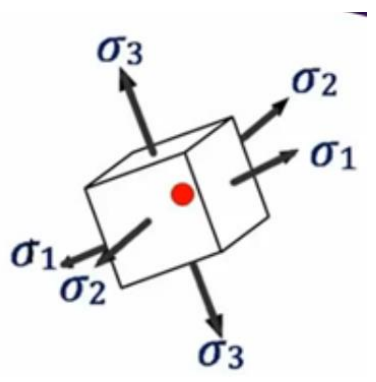


The concept of Von Mises Stress arises from distortion energy failure theory. Based on this theory, failure occurs when the distortion energy per unit volume in actual case is more than the distortion energy per unit volume for yield in a simple tension case at the time of failure.

$$
\left[\frac{\left(\sigma_{1}-\sigma_{2}\right)^{2}+\left(\sigma_{2}-\sigma_{3}\right)^{2}+\left(\sigma_{3}-\sigma_{1}\right)^{2}}{2}\right]^{1 / 2} \geq \sigma_{y}
$$

Bone and suture tissues within the infant's skull undergo ductile behavior due to the stresses applied during sleep or cranial helmet therapy. Therefore, although failure of these tissues within the infant's skull is not a point of interest in this study, the concept of Von Mises stress can still be applied for quantifying the complex stress distribution within the skull system since the quantity is a representation of the principal stresses in all three directions.

\section{Methods}

\section{Model Preparation}

The laser-scanned 3D surface contours of a male subject diagnosed with PP were obtained before and after three months of cranial molding therapy at Hanger Clinic, San Luis Obispo. The patient was five months old when treatment was initiated. Figure 10 demonstrates the results after three months of cranial molding therapy. Areas shown in red color represent the scanned contour after, and areas in green refer to the head contour before treatment. 


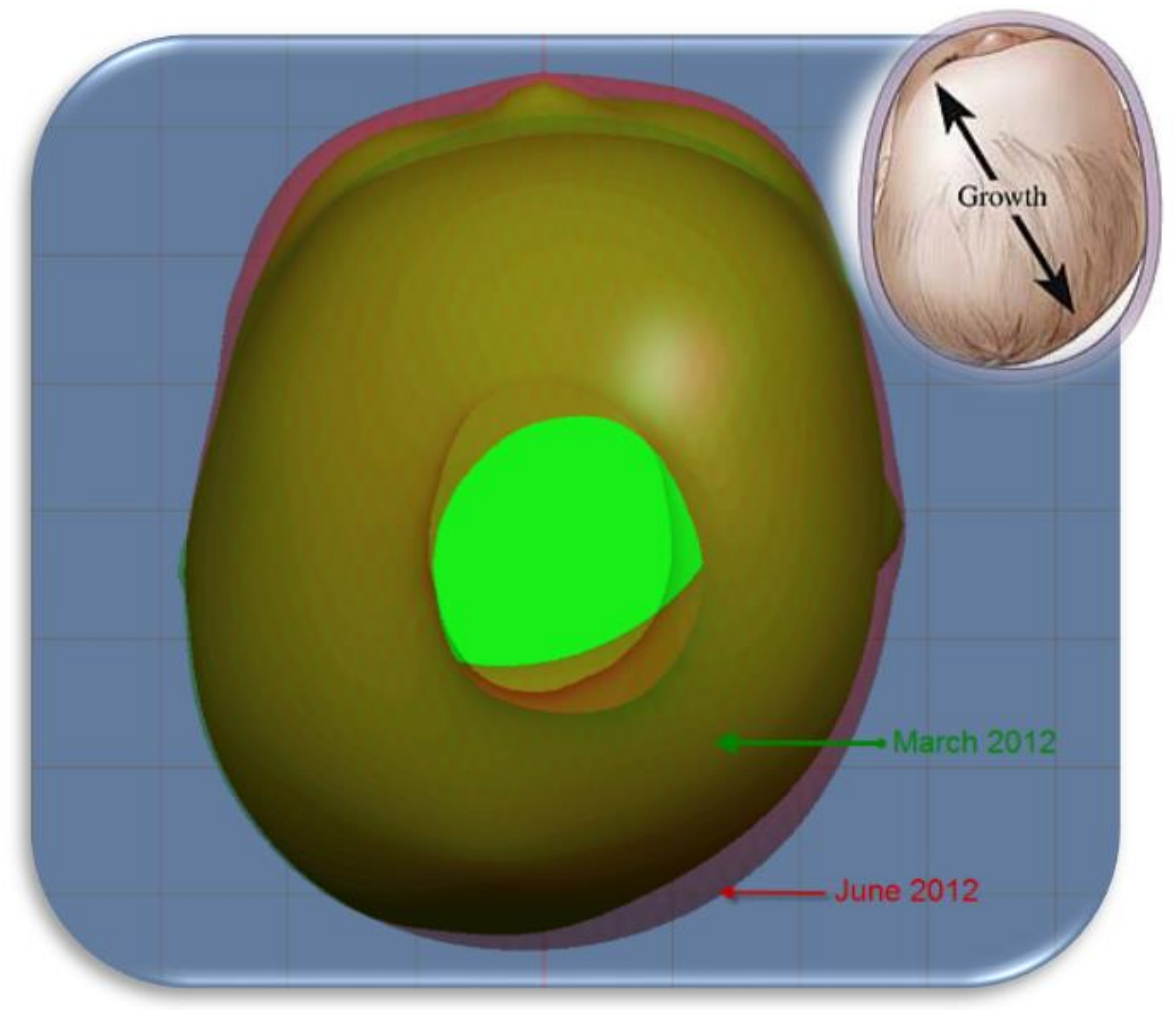

Figure 10- Effect of the cranial molding treatment on correcting the patient's head asymmetry: Compares before (March 2012) and after (June 2012) treatment head contours.
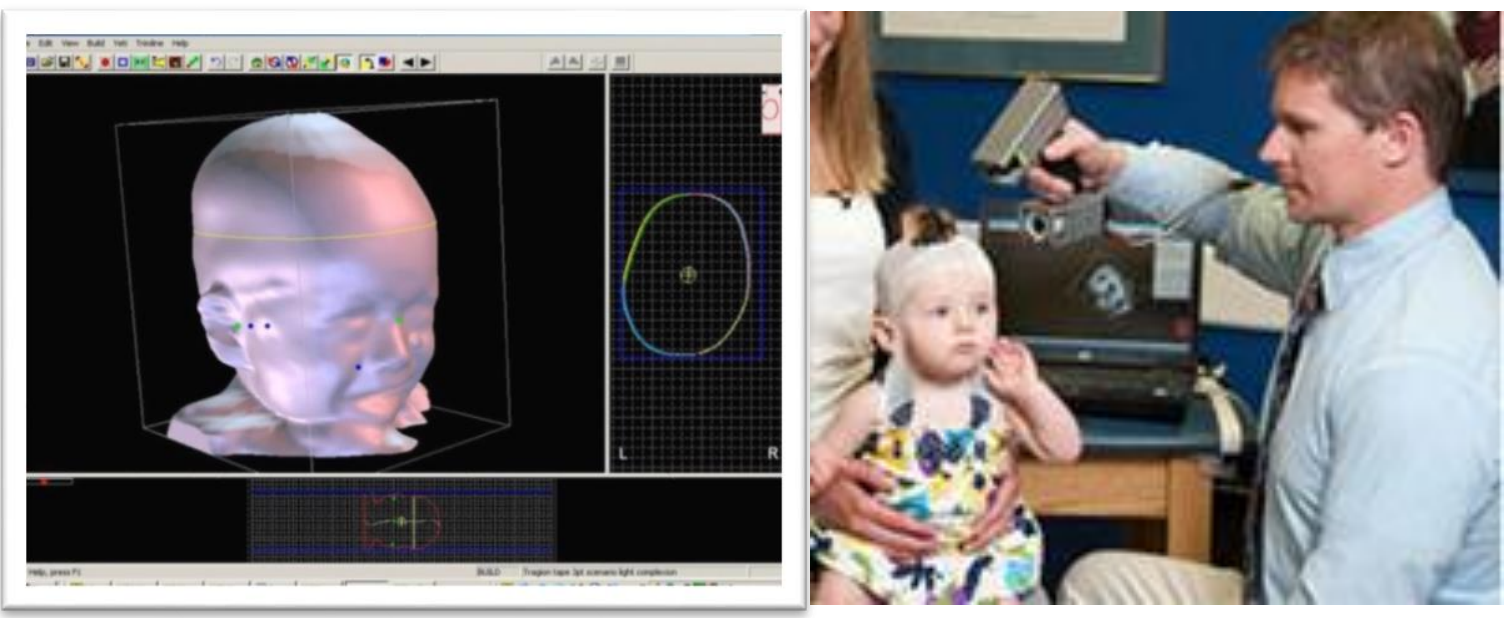

Figure 11- Insignia's 3D laser scanner technology at Hanger Clinic (25).

The laser scanning files were generated in Standard Tessellation Language (STL) format with about 28000 triangular faces in each file that defined the surface geometry of the subject's 
head. As you can see from Figure 12, the original STL file for the "before treatment scan" has two open surfaces at the top and bottom of the geometry. In order to be able to use the files in Abaqus software and perform FEA, they had to be converted to Initial Graphics Exchange Specification (IGES) format with an enclosed geometry (no open boundaries within the surface). Therefore, they had to undergo a series of modifications before they could be used for FEA.
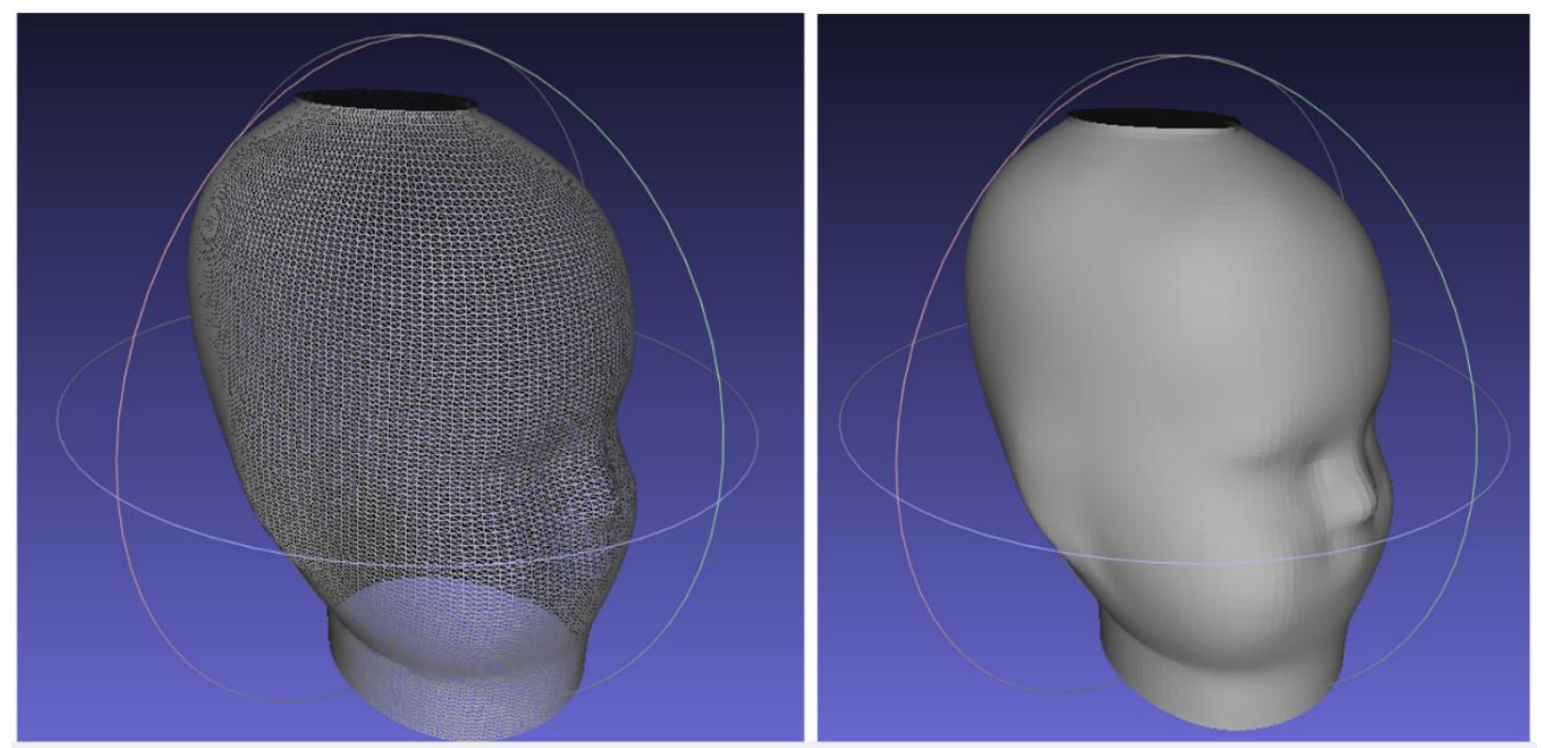

Figure 12- The original STL file of the patient's scanned head surface contour: Contains 28000 triangles.

Each STL file was opened in MeshLab software, the geometry was re-meshed, and the number of triangular faces was brought down to 6000 so that the resulting STL file could be opened by SolidWorks for further preparation of the file. The result of this process is shownin Figure 13. 

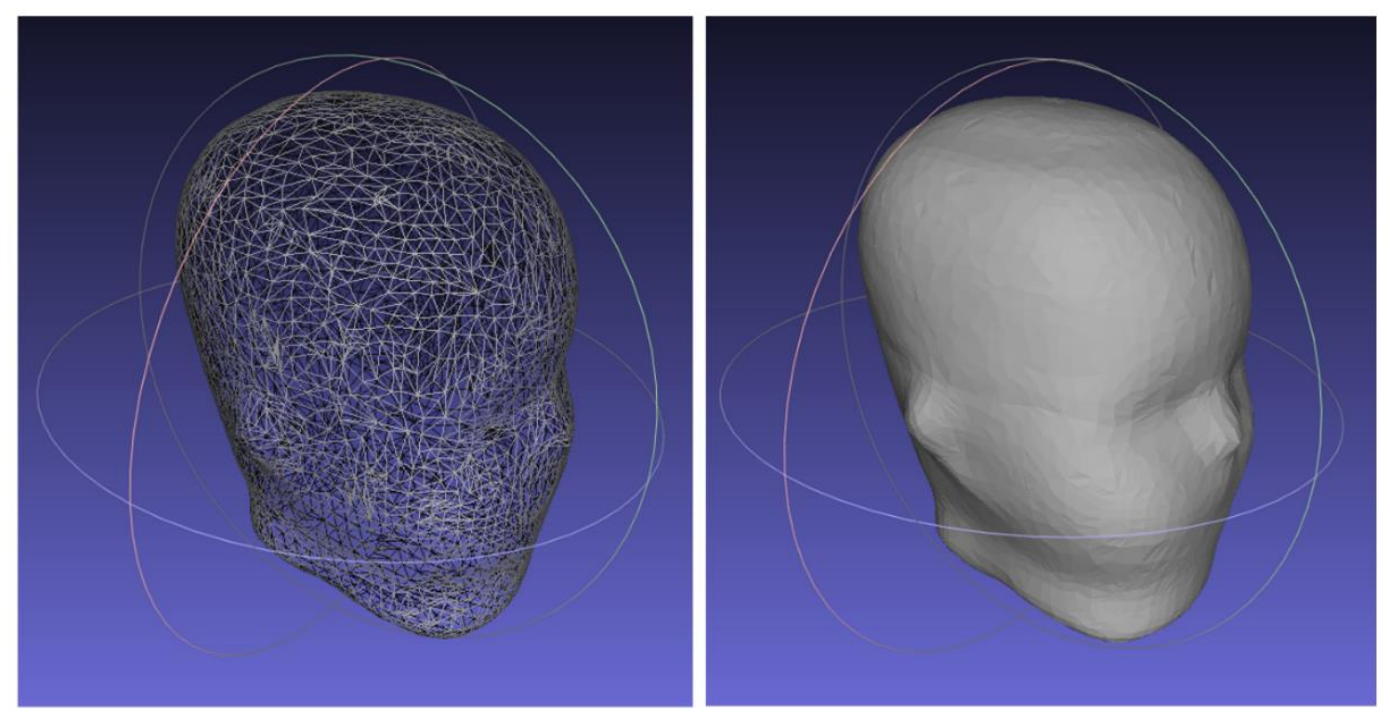

Figure 13- The modified water-tight STL file of the patient's scanned head surface contour with 6000 triangles.

In SolidWorks, the files were scaled properly to convert the units from millimeters to inches, and the suture lines were traced over the surface of the part according to a CT scan image of an infant's skull. Tracing the suture boundaries would allow us to separate the skull suture sections from bone sections and assign different material properties to different areas of the model in the Abaqus environment.

The file was opened in Abaqus 6.12, and the faulty faces were repaired using the repair tools within the software. Then the mesh resulting from the triangular faces of the STL was deleted, and the part was re-meshed using the mesh options in Abaqus. Figure 14 shows the CT scanned image as well as the re-meshed part with suture areas highlighted in red. The mesh characteristics and section properties for each of the three models will be further discussed later throughout this section. 


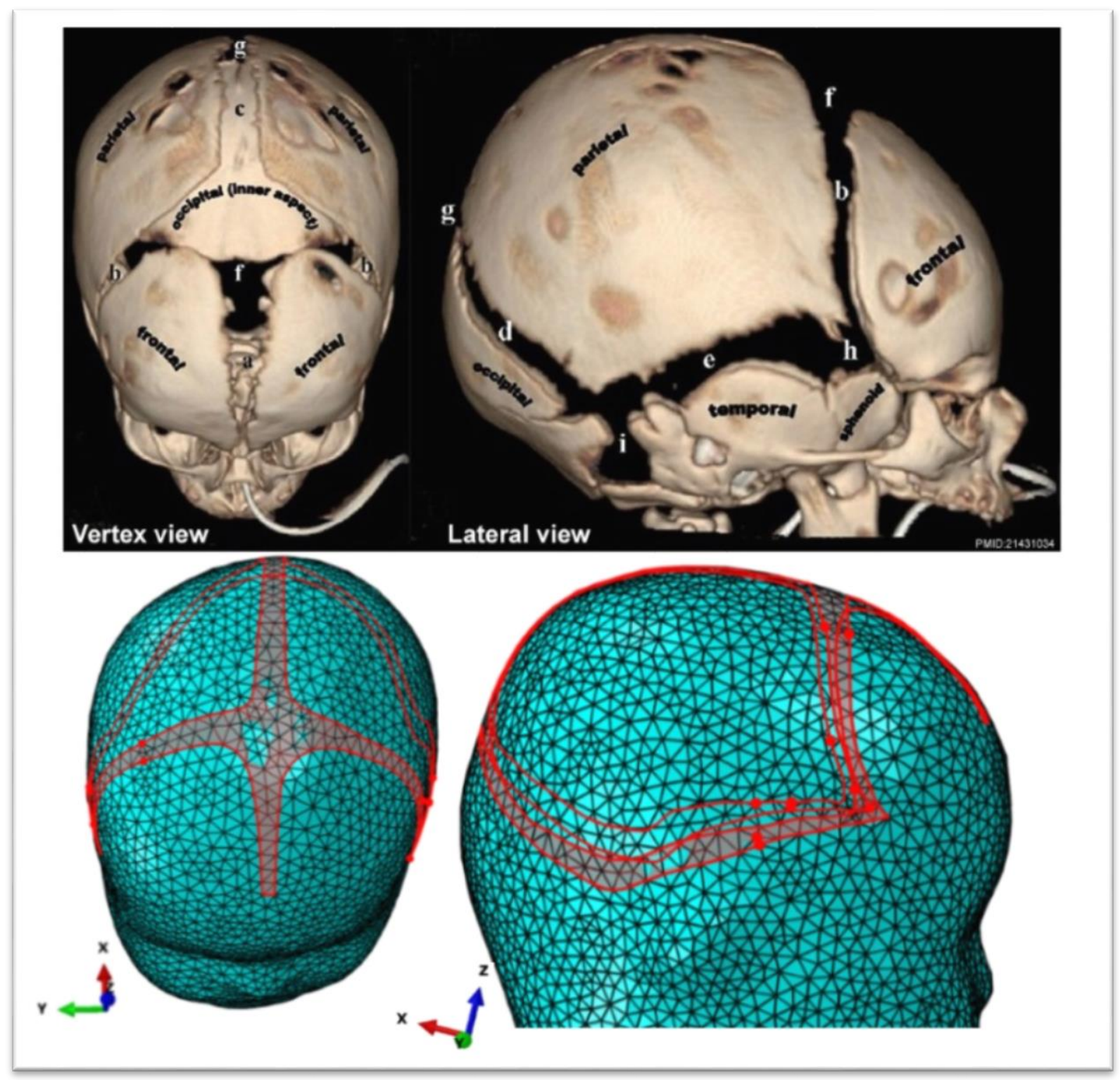

Figure 14- Tracing cranial sutures over the modified 3D computer image according to the $C T$ scanned images (5).

The cranial helmet was constructed using the STL file of the cranial cavity. The file was prepared for being imported into SolidWorks in a similar fashion as the "before scan" file. The geometry was then trimmed and modified to create the helmet. Figure 15 contrasts theconstructed helmet with the resurfaced cranial molding cavity file. The file was then saved as an IGES file and imported into Abaqus. 

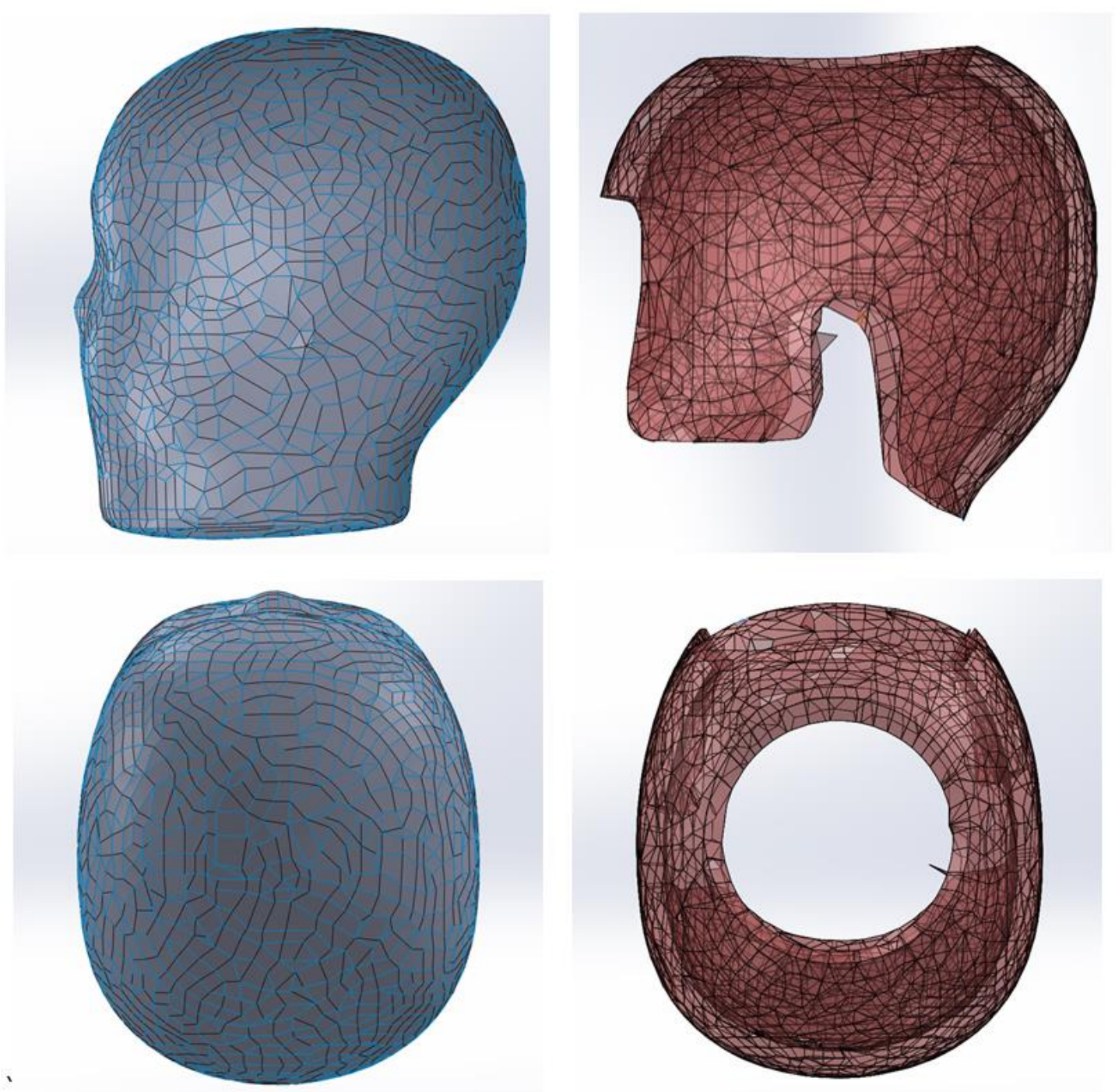

Figure 15- Creating the 3D model of the orthotic helmet: Reconstructed based on the helmet cavity file provided by Hanger Clinic.

Lastly, a solid model of a flat 12"x7"x 2" rectangular pillow was drawn in SolidWorks and imported into Abaqus as a solid object. As you can see from Figure 16, small sections have been created in this model to allow for meshing in the Abaqus environment. 


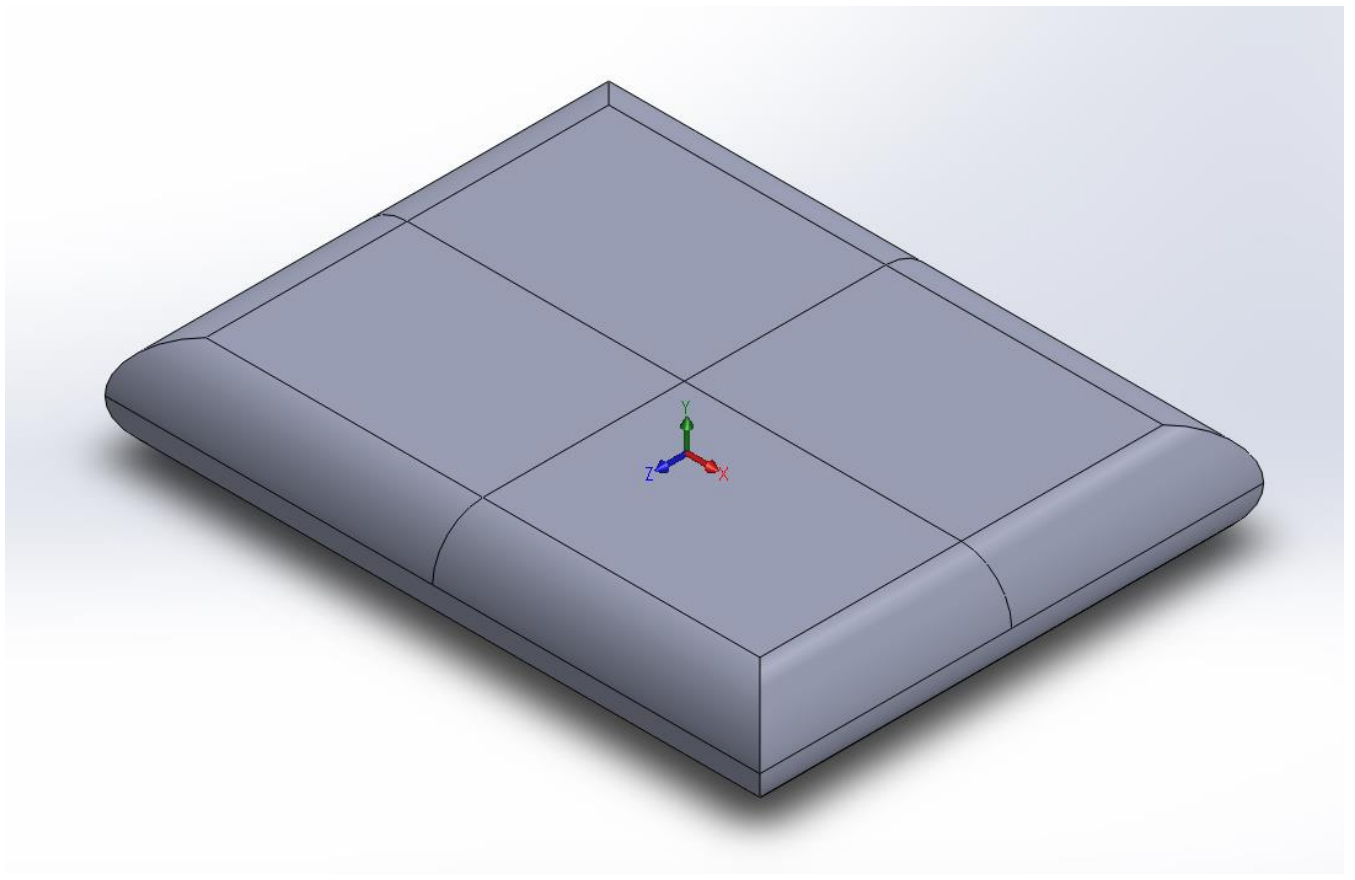

Figure 16- Solid model of the pillow used to create the FE models.

The four prepared files of the baby with the deformed shape, the cranial molding cavity representing a healthy head shape, the cranial helmet, and the pillow were used in development of the three separate models:

- Model 1: A healthy baby sleeping on a pillow in supine position

- Model 2: The PP patient sleeping on a pillow in supine position with the flat area touching the surface of the pillow

- Model 3: The PP subject sleeping in supine position wearing the cranial helmet

Considering that the average weight of a 5 months old baby is about $16.5 \mathrm{lbs}$, and the head of a 5 months old infant is about $1 / 4$ of its total weight, the weight of the baby's head was estimated to be about $4.125 \mathrm{lbs}$ in all three models $(26,27)$. 
Model 1: A Healthy Baby Sleeping on a Pillow in Supine Position

The healthy head contour and the pillow were imported and assembled in Abaqus 6.12 to represent a baby sleeping in supine position (Figure 17).
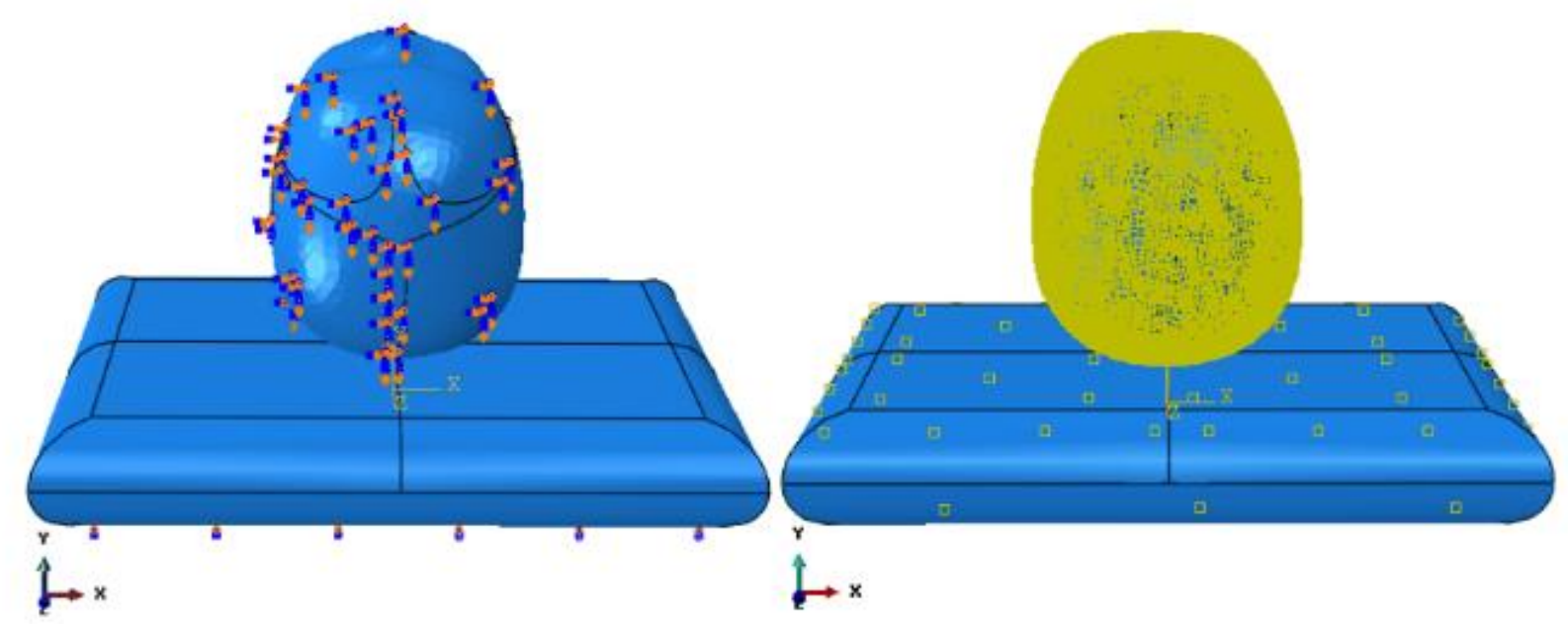

Figure 17- The loading, boundary, and surface contact conditions for model 1.

The loading and boundary conditions for this model are listed below:

- Weight of the head: $4.125 \mathrm{lbs}(26,27)$

- Bottom of the pillow was fixed in $x, y$, and $z$ planes

- Head was allowed to only dislocate in y direction

- Finite-sliding normal hard contact between the head and the top surface of the pillow.

- Element type: Quadratic Triangular Shell Element.

Model 2: The PP Patient Sleeping on a Pillow in Supine Position

The PP head contour and the pillow were imported and assembled in Abaqus 6.12 to represent a baby diagnosed with PP sleeping in supine position (Figure 18). 

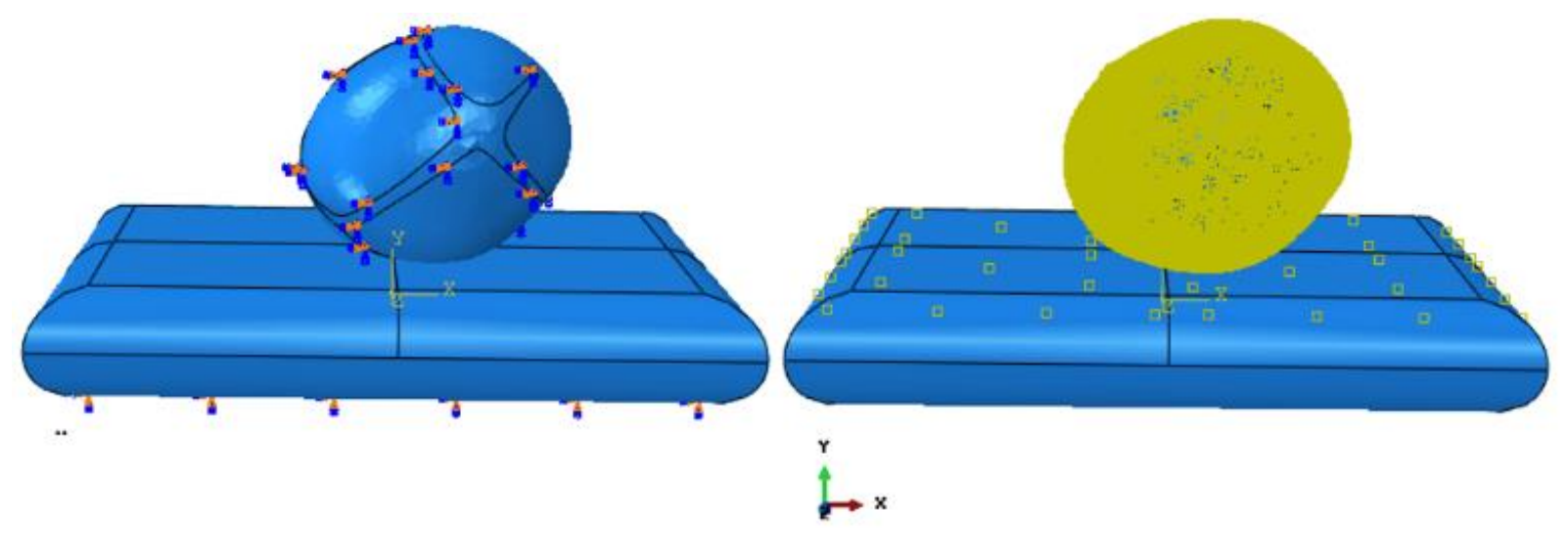

Figure 18- The loading, boundary, and surface contact conditions for model 2.

The loading and boundary conditions for this model are listed below:

- Weight of the head: $4.125 \mathrm{lbs}(26,27)$

- Bottom of the pillow was fixed in $x, y$, and $z$ planes

- Head was allowed to only dislocate in y direction

- Finite-sliding normal hard contact between the head and the top surface of the pillow.

- Element type: Quadratic Triangular Shell Element.

Model 3: The PP Subject Sleeping in Supine Position Wearing the Cranial Helmet

The PP head contour and the cranial helmet were imported and assembled in Abaqus 6.12 to represent a baby sleeping in supine position wearing a helmet (Figure 19). 

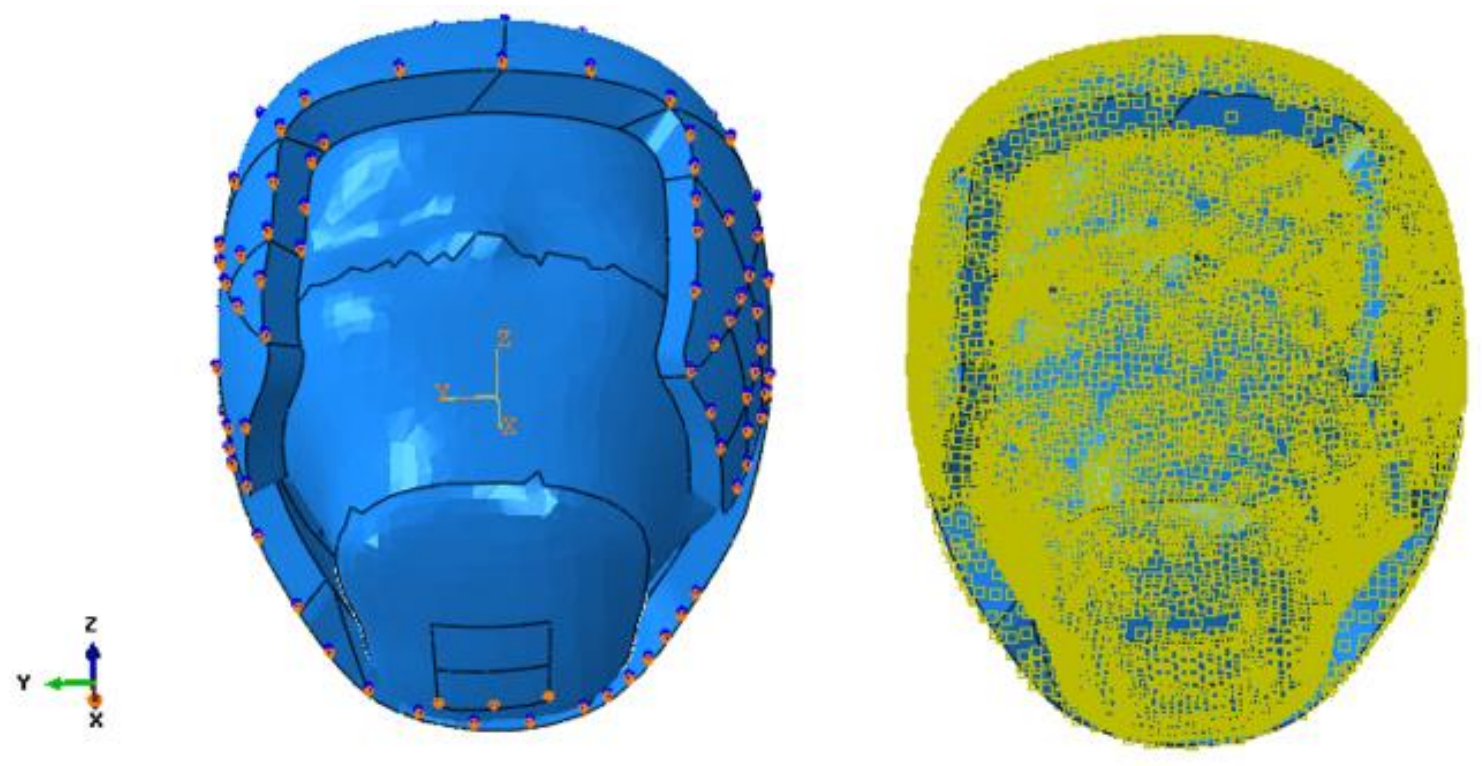

Figure 19- The loading, boundary, and surface contact conditions for model 3.

The loading and boundary conditions for this model are listed below:

- Weight of the head: $4.125 \mathrm{lbs}(26,27)$

- The ABS plastic on the outer layer of the helmet was constrained to have no displacement or rotations in any directions.

- Two points below the neck area (where spinal cord would be) were fixed as hinge joints that would only allow the head to rotate around y-axis.

- Finite-sliding normal hard contact between the head and the helmet.

Assumptions for all Three Models:

- Uniform skull thickness of $3 \mathrm{~mm}$ everywhere within the model

- Isotropic material properties

- Effects of cranial pressure, atmospheric pressure, weight of the helmet, soft tissues, bone modeling, and bone remodeling are minimal and negligible 
- Material properties are not rate dependent

- Frictionless tangential contact between the parts

- Hard normal contact between the parts

Table 1 summarizes material properties assigned to different sections of all three models (ABS plastic is only used in model 3).

\begin{tabular}{|l|c|c|c|}
\hline Materials & $\begin{array}{c}\text { Young's Modulus } \\
\text { (PSI) }\end{array}$ & $\begin{array}{c}\text { Poisson's } \\
\text { Ratio }\end{array}$ & $\begin{array}{c}\text { Element } \\
\text { Thickness (in) }\end{array}$ \\
\hline ABS Plastic & $362594.25(28,29)$ & 0.3 & 0.1 \\
\hline Polyethylene Foam & $174.05(30,31)$ & -0.3 & 0.5 \\
\hline Parietal and Occipital Bones & $56461.0(18)$ & 0.3 & 0.118 \\
\hline Sutures & $1176.3(19)$ & 0.3 & 0.118 \\
\hline
\end{tabular}

Table 2- Material properties used for the helmet, bones, sutures, and the pillow.

The material properties for the infant's skull were determined from a study performed by B. Coats et al. (8). Human infant ( $<1$ year old) cranial bone and suture from 23 skulls were tested in three-point bending and tension at rates ranging from $1.2-2.8 \mathrm{~m} / \mathrm{s}$ to find the elastic modulus of each of the samples from different age groups at different rates (8). The average elastic moduli from 25 occipital bone samples and 21 parietal bone samples were taken and assigned to the bone regions of the models. The average elastic moduli for 10 suture samples were taken and assigned to the suture regions of the models.

\section{Mesh Convergence}

Mesh convergence plots were used to confirm that the number of elements used to run the model were sufficient for converging to a specific value. The nodes shown by the red arrow in Figures 20, 21, and 22 were used for collecting the mesh convergence data. These specific nodes 
were chosen because their location stayed the same regardless of the seed size in all three models.

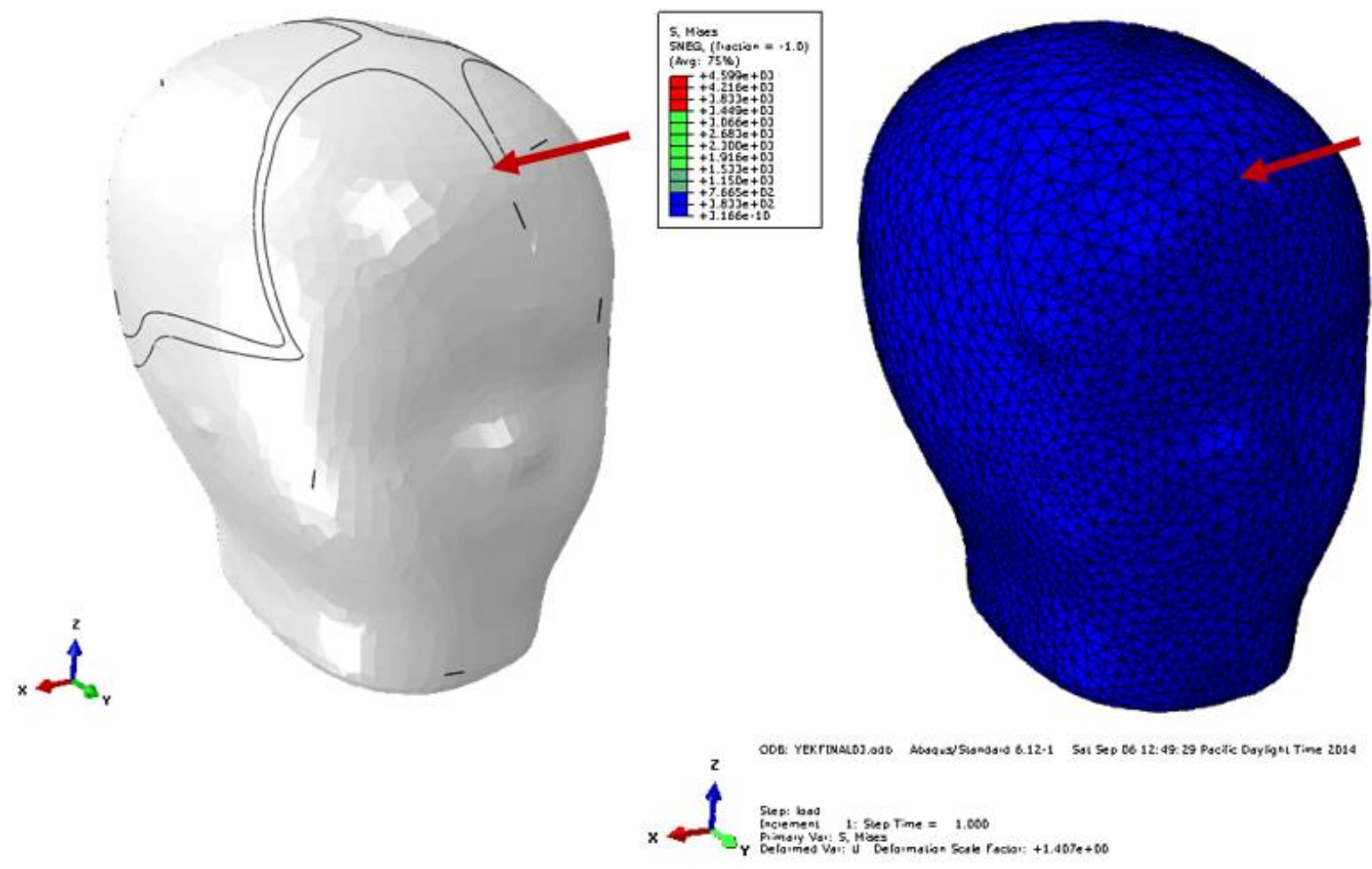

\begin{tabular}{|c|c|c|}
\hline \multicolumn{3}{|c|}{ Model 1 } \\
\hline $\begin{array}{c}\text { Seed } \\
\text { Size (in) }\end{array}$ & $\begin{array}{c}\text { Number of } \\
\text { Elements }\end{array}$ & $\begin{array}{c}\text { Node } \\
\text { Value (PSI) }\end{array}$ \\
\hline 0.25 & 138312 & 0.12 \\
\hline 0.4 & 64245 & 0.12 \\
\hline 0.5 & 25530 & 0.12 \\
\hline 1 & 6935 & 0.09 \\
\hline 2 & 2746 & 0.09 \\
\hline 3 & 1645 & 0.10 \\
\hline
\end{tabular}

\section{Mesh Convergence Plot for Model 1}

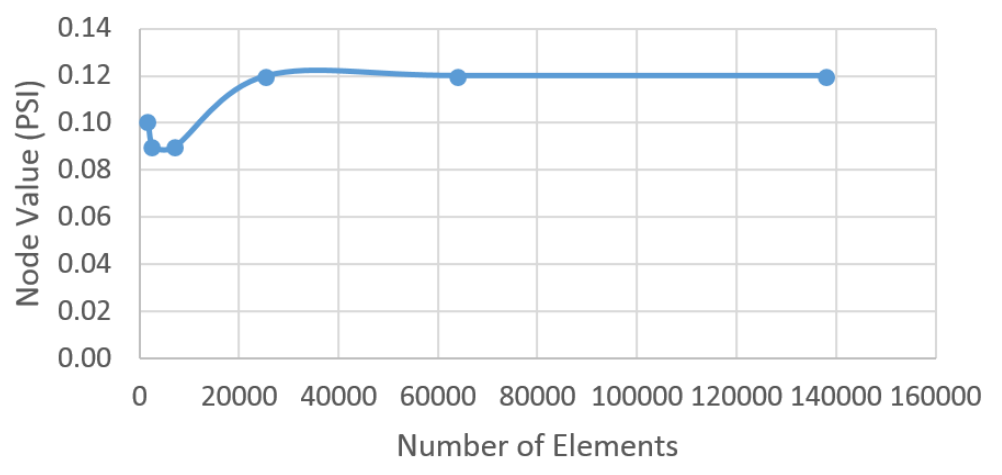

Figure 20-Mesh convergence plot of Model 1: The convergence values shown in the table. The red arrow points to the node used for generating the plot. 

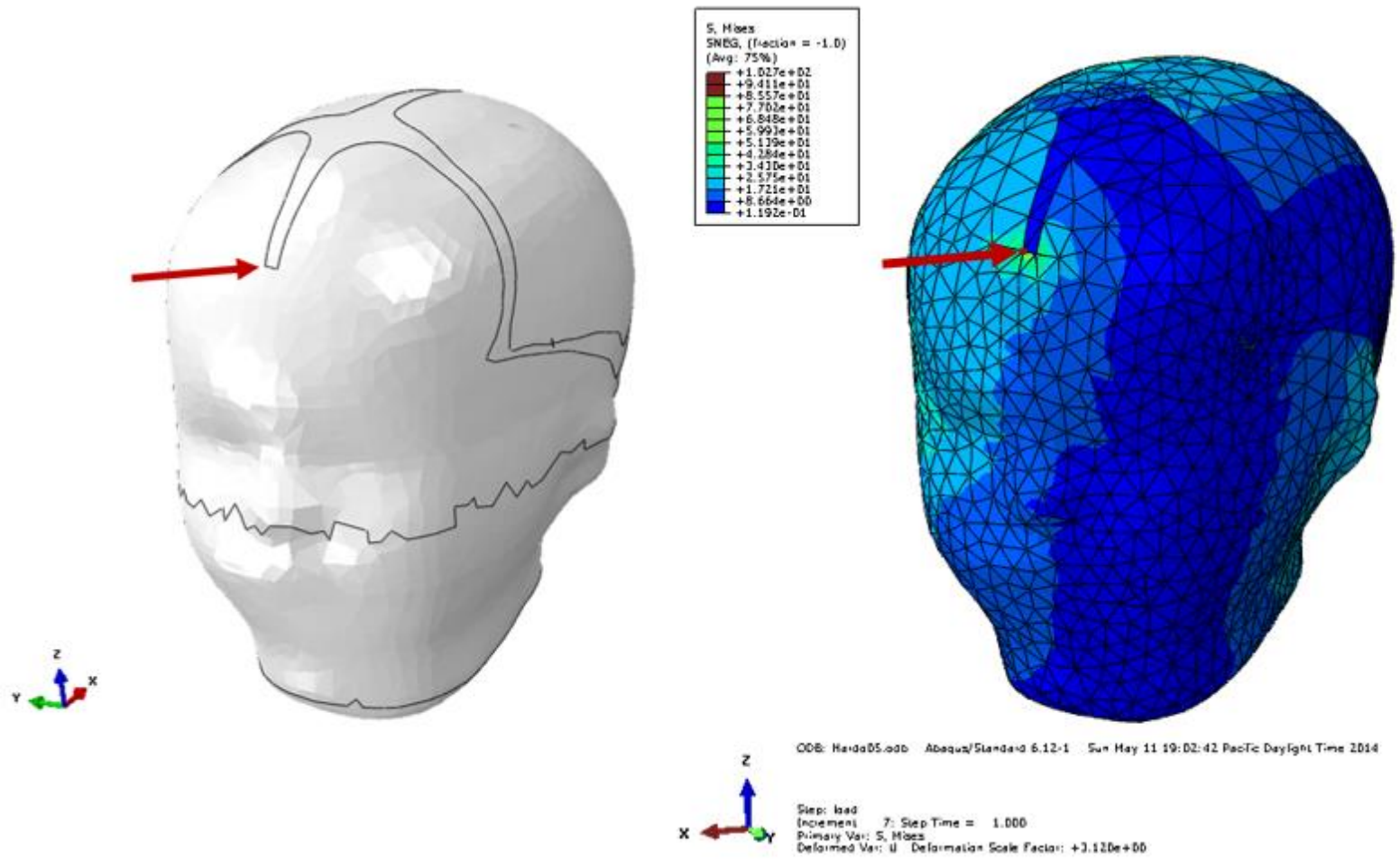

\begin{tabular}{|c|c|c|}
\hline \multicolumn{3}{|c|}{ Model 2 } \\
\hline $\begin{array}{c}\text { Seed } \\
\text { Size (in) }\end{array}$ & $\begin{array}{c}\text { Element } \\
\text { Number }\end{array}$ & $\begin{array}{c}\text { Node } \\
\text { Value (PSI) }\end{array}$ \\
\hline 0.2 & 240044 & 0.22 \\
\hline 0.3 & 74529 & 0.24 \\
\hline 0.4 & 38409 & 0.25 \\
\hline 0.5 & 22968 & 0.24 \\
\hline 1 & 4417 & 5.19 \\
\hline 2 & 1964 & 0.24 \\
\hline 3 & 1165 & 0.02 \\
\hline
\end{tabular}

\section{Mesh Convergence Plot for Model 2}

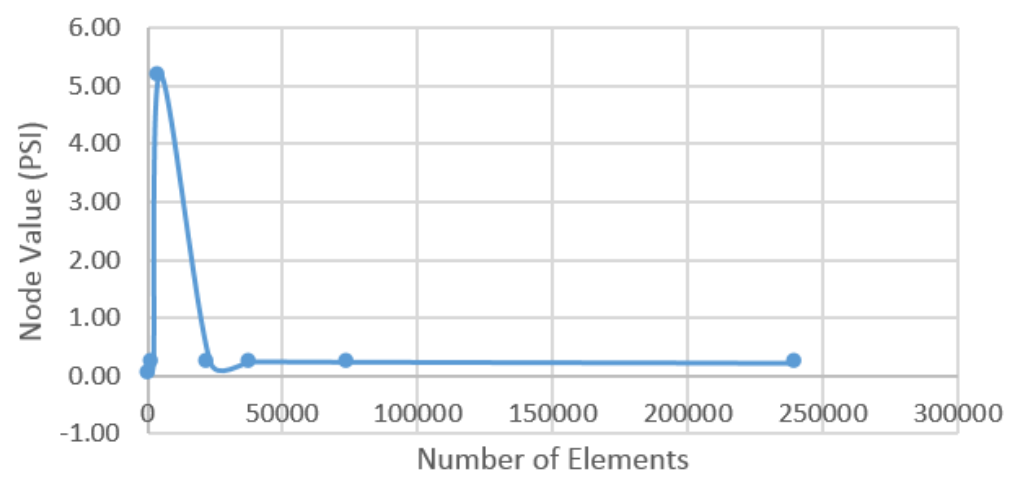

Figure 21-Mesh convergence plot of Model 2: The convergence values shown in the table. The red arrow points to the node used for generating the plot. 


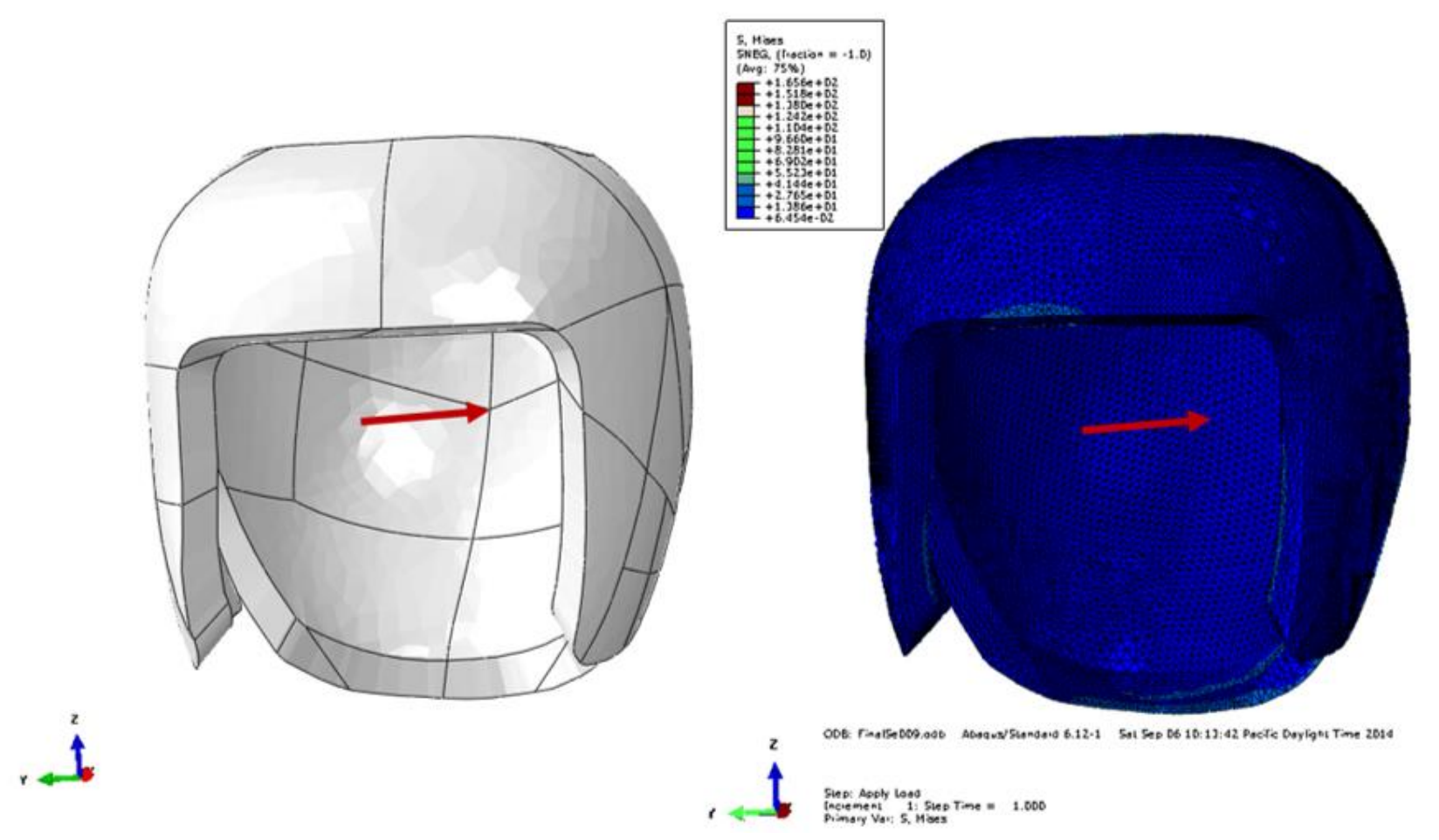

\begin{tabular}{|c|c|c|}
\hline \multicolumn{3}{|c|}{ Model 3 } \\
\hline $\begin{array}{c}\text { Seed } \\
\text { Size (in) }\end{array}$ & $\begin{array}{c}\text { Element } \\
\text { Number }\end{array}$ & $\begin{array}{c}\text { Node } \\
\text { Value (PSI) }\end{array}$ \\
\hline 0.09 & 80734 & 0.0075 \\
\hline 0.12 & 47088 & 0.0076 \\
\hline 0.2 & 18288 & 0.0077 \\
\hline 0.5 & 5250 & 0.0090 \\
\hline 1 & 3448 & 0.0056 \\
\hline
\end{tabular}

\section{Mesh Convergence Plot for Model 3}

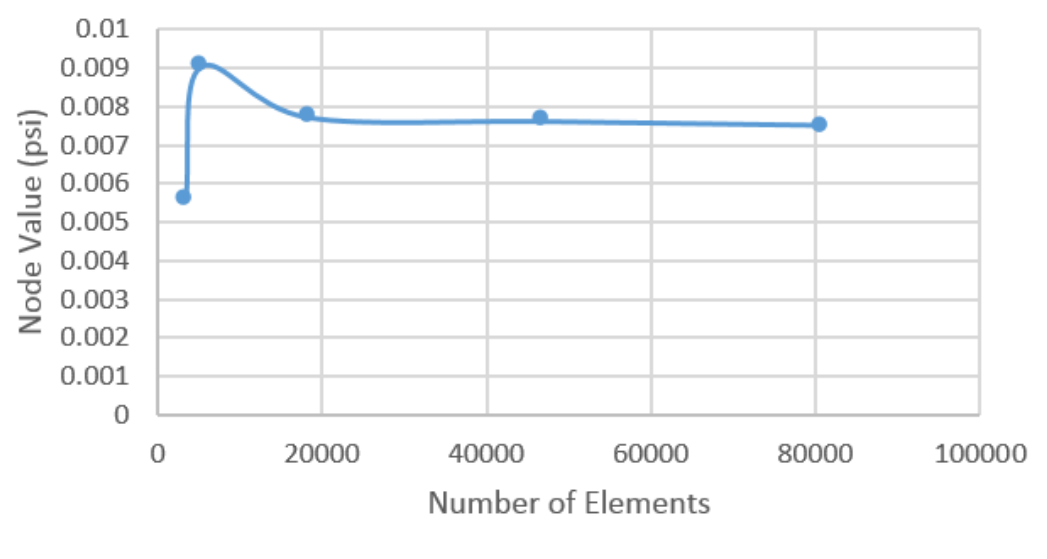

Figure 22- Mesh convergence plot of Model 3: The convergence values shown in the table. The red arrow points to the node used for generating the plot. 
Results

Figure 23 shows a color contour plot of the Von Mises stress distribution throughout the isolated suture segments of model 1 . Areas in red represent high stress locations and areas in blue correspond to low stress locations.
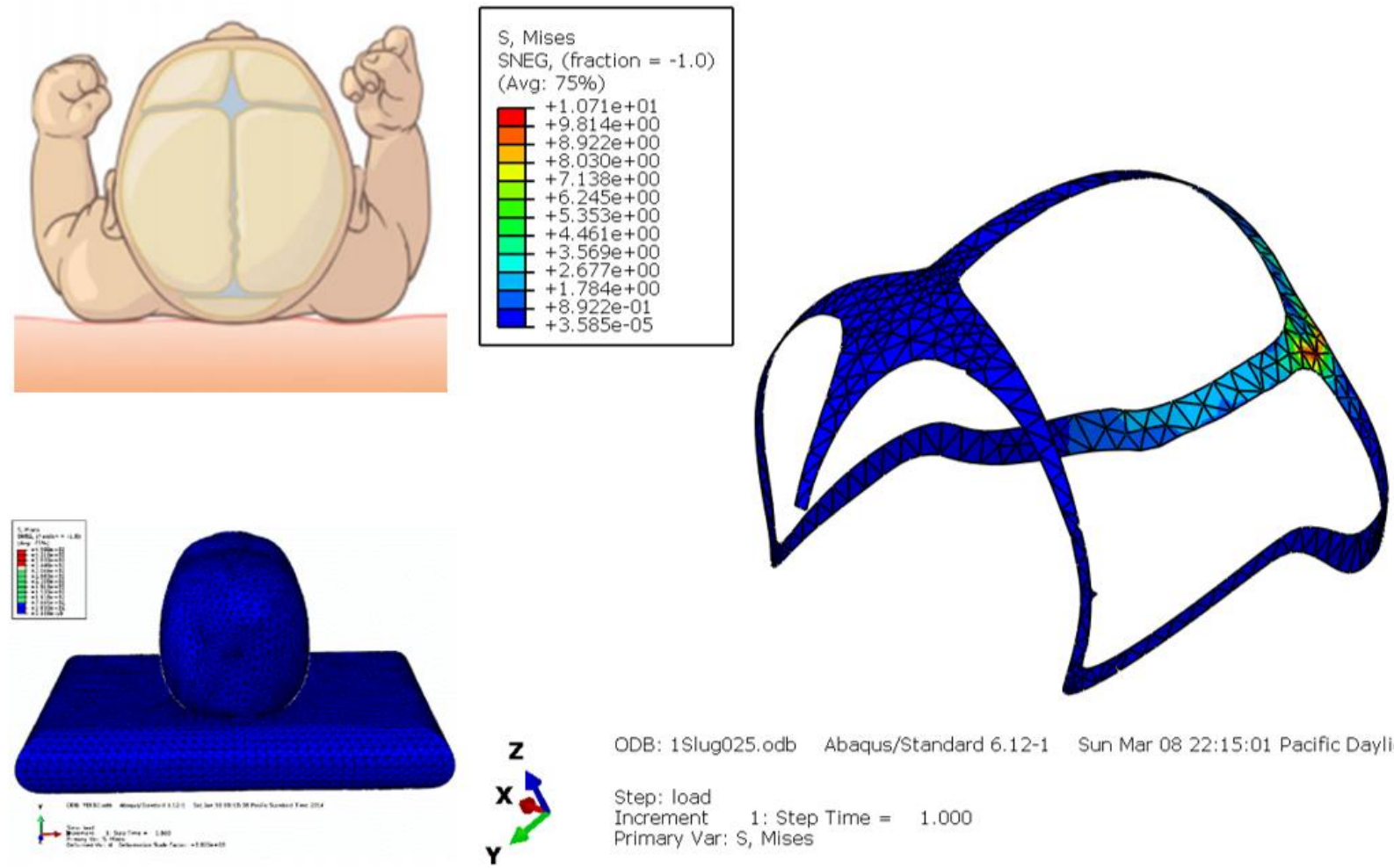

ODB: 1 Slug025.odb Abaqus/Standard 6.12-1 Sun Mar 08 22:15:01 Pacific Daylight Time 2015
Step: load
Increment $\quad$ 1: Step Time $=1.000$
Primary Var: S, Mises

Figure 23- Color contour plot of Von Mises stress distribution for model 1: baby with a healthy skull sleeping in supine position on a pillow.

Table 3 summarizes the number of elements, minimum, maximum, and average stress values for each of the 13 suture segments. It may be noted that the posterior fontanelle has the highest stress values among all suture groups of this model. 


\begin{tabular}{|lcc|cc|}
\hline Suture Section & $\begin{array}{c}\text { Number of } \\
\text { Integration points }\end{array}$ & Min (PSI) & $\begin{array}{c}\text { Max } \\
\text { (PSI) }\end{array}$ & $\begin{array}{c}\text { Average } \\
\text { (PSI) }\end{array}$ \\
\hline Anterior Fontanelle and & 474 & 0.00 & 0.09 & 0.02 \\
\hline Frontal Suture & & & & \\
\hline Sagittal Suture & 339 & 0.00 & 4.59 & 0.51 \\
\hline Posterior Fontanelle & 156 & 0.24 & 10.51 & 3.32 \\
\hline Left Corronal Suture & 90 & 0.00 & 0.01 & 0.00 \\
\hline Right Corronal Suture & 72 & 0.00 & 0.01 & 0.00 \\
\hline Left Sphenoid Fontanelle & 147 & 0.00 & 0.25 & 0.062 \\
\hline Right Sphenoid Fontanelle & 117 & 0.00 & 0.32 & 0.03 \\
\hline Left Squamosal Suture & 42 & 0.12 & 0.46 & 0.28 \\
\hline Right Squamosal Suture & 42 & 0.15 & 0.65 & 0.43 \\
\hline Left Mastoid Fontanelle & 57 & 0.38 & 0.64 & 0.53 \\
\hline Right Mastoid Fontanelle & 60 & 0.23 & 0.85 & 0.45 \\
\hline Left Lamboidal Suture & 72 & 0.56 & 0.95 & 0.74 \\
\hline Right Lamboidal Suture & 96 & 0.72 & 2.38 & 1.69 \\
\hline
\end{tabular}

Table 3- Stress Value Results for Model 1: Number of elements, minimum, maximum, and average Von Mises stress values off all 13 different suture sections for the baby with a healthy skull sleeping in supine position on a pillow.

Similarly, figures 24 and 25 show the color contour plots of Von Mises stress distributions, and tables 4 and 5 summarize the number of elements, minimum, and maximum stress values in different suture sections of models 2 and 3 respectively. Highest stress values in model 2 are the right sphenoid fontanelle, sagittal, and the right coronal sutures, and highest stress values in model 3 are found in the left sphenoid fontanelle and the left squamosal suture. For detailed contour plots of each of the suture segments for all three models please refer to the Appendix. 


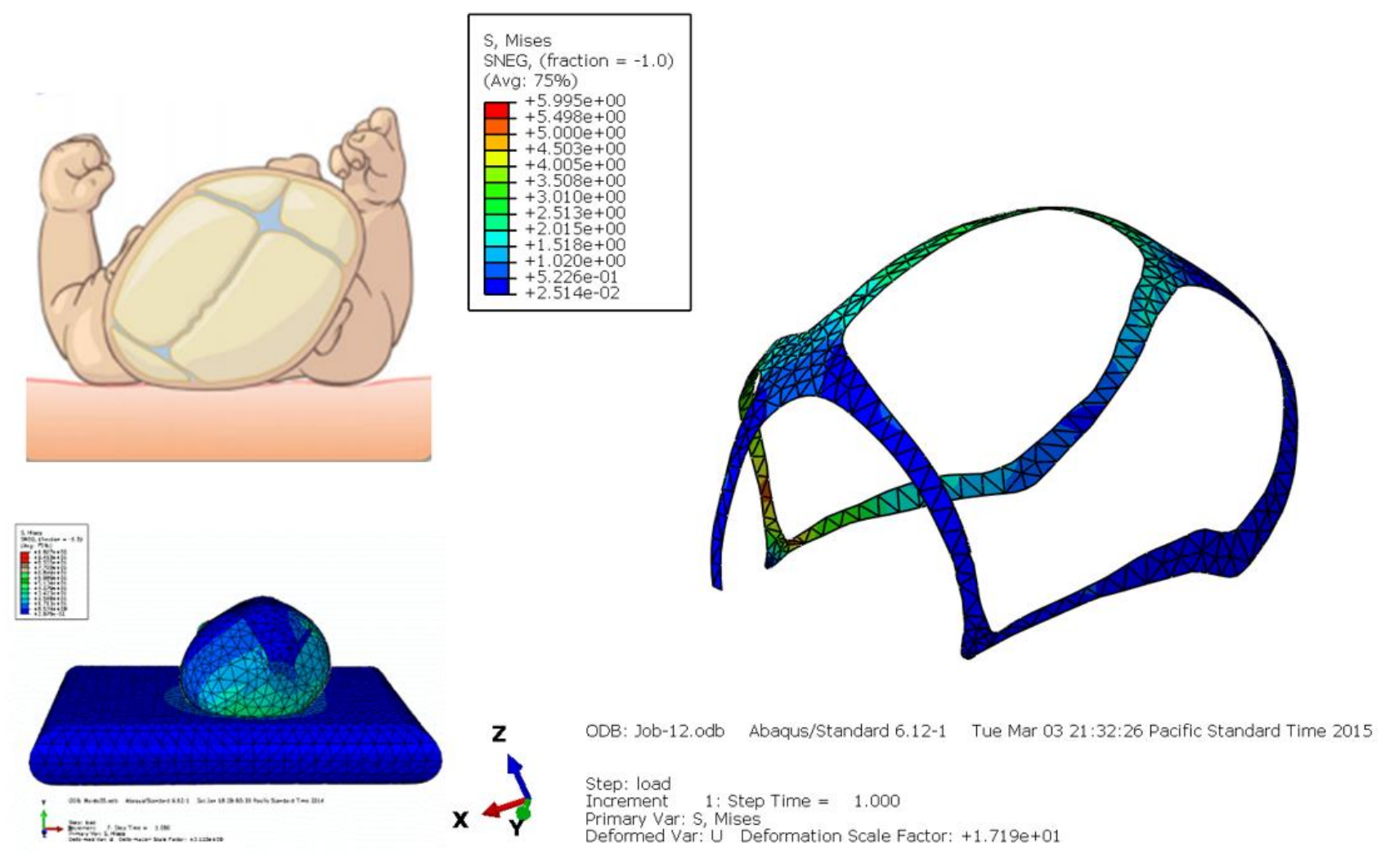

Figure 24- Color contour plot of Von Mises stress distribution for model 2: PP patient sleeping in supine position on the flat side of his head on a pillow.

\begin{tabular}{|c|c|c|c|c|}
\hline Suture Section & $\begin{array}{c}\text { Number of } \\
\text { integration points }\end{array}$ & Min (PSI) & $\begin{array}{l}\text { Max } \\
\text { (PSI) }\end{array}$ & $\begin{array}{l}\text { Average } \\
\text { (PSI) }\end{array}$ \\
\hline $\begin{array}{l}\text { Anterior Fontanelle and Frontal } \\
\text { Suture }\end{array}$ & 285 & 0.22 & 2.31 & 0.93 \\
\hline Sagittal Suture & 162 & 1.65 & 3.12 & 2.50 \\
\hline Posterior Fontanelle & 153 & 0.08 & 2.85 & 1.24 \\
\hline Left Corronal Suture & 69 & 0.24 & 0.55 & 0.40 \\
\hline Right Corronal Suture & 102 & 1.49 & 4.17 & 2.43 \\
\hline Left Sphenoid Fontanelle & 108 & 0.02 & 0.55 & 0.26 \\
\hline Right Sphenoid Fontanelle & 111 & 0.41 & 5.67 & 3.46 \\
\hline Left Squamosal Suture & 60 & 0.24 & 0.54 & 0.37 \\
\hline Right Squamosal Suture & 54 & 1.06 & 3.42 & 1.80 \\
\hline Left Mastoid Fontanelle & 87 & 0.08 & 0.52 & 0.32 \\
\hline Right Mastoid Fontanelle & 57 & 0.16 & 1.50 & 0.82 \\
\hline Left Lamboidal Suture & 66 & 0.27 & 0.53 & 0.45 \\
\hline Right Lamboidal Suture & 81 & 0.07 & 1.92 & 1.14 \\
\hline
\end{tabular}

Table 4- Stress Value Results for Model 2: Number of elements, minimum, maximum, and average Von Mises stress values off all 13 different suture sections for PP patient sleeping in supine position on the flat side of his head on a pillow. 

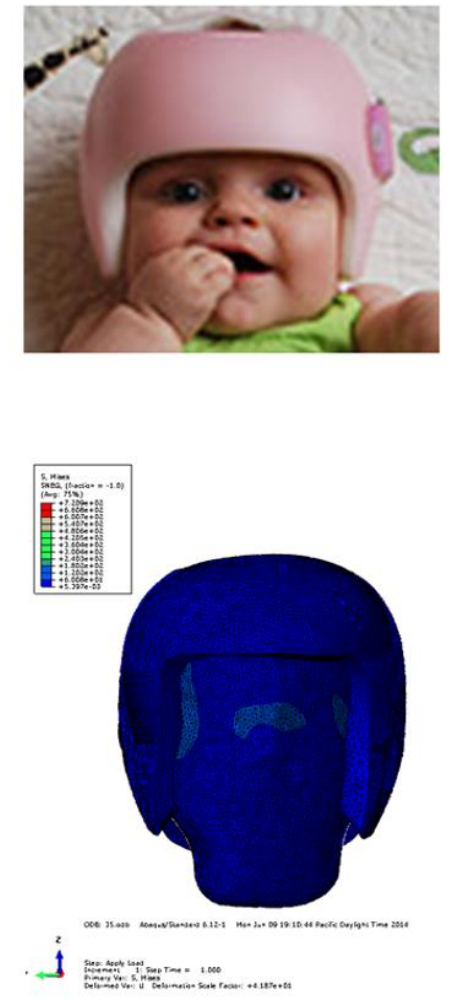
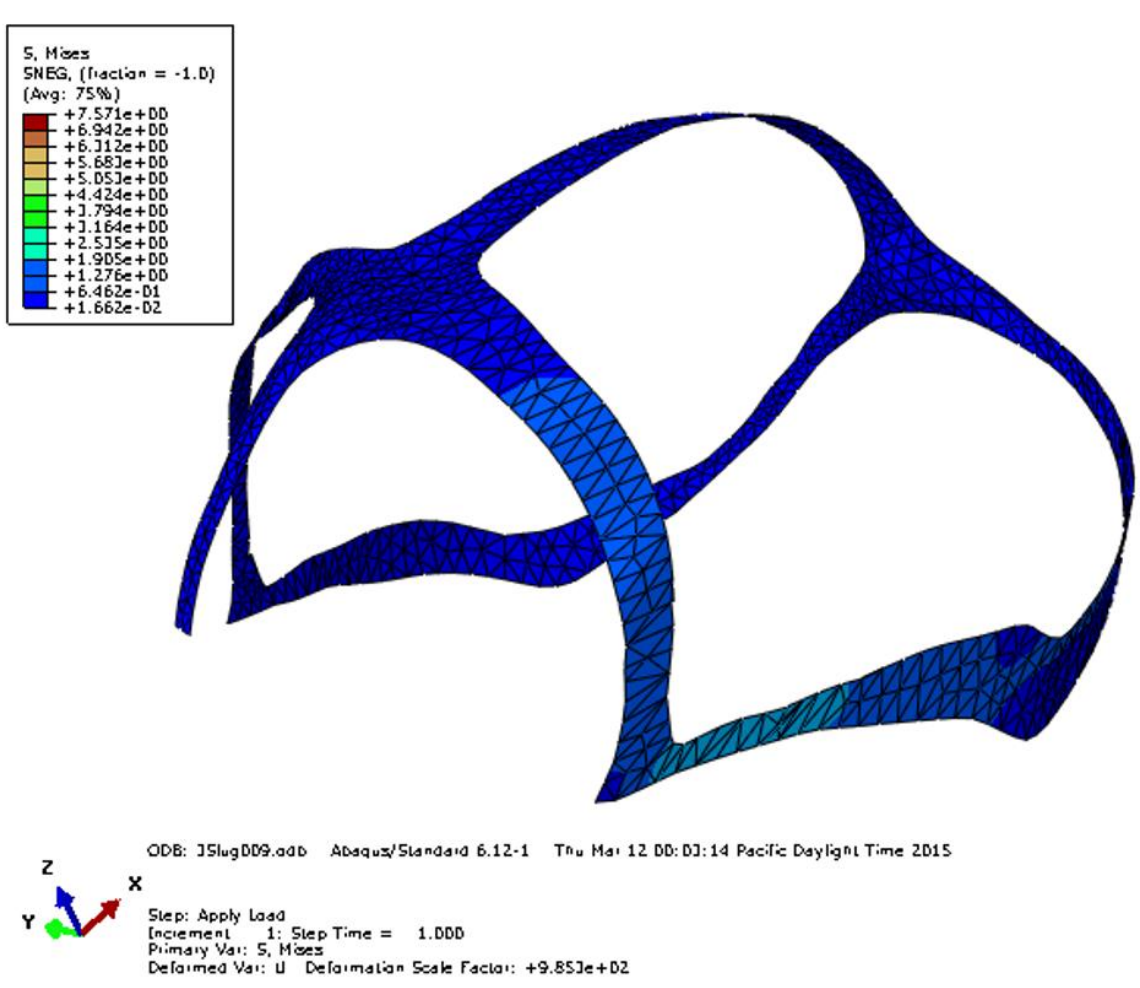

Figure 25- Color contour plot of Von Mises stress distribution for model 3: PP patient sleeping in supine position wearing an orthotic helmet.

\begin{tabular}{|c|c|c|c|c|}
\hline Suture Section & $\begin{array}{l}\text { Number of } \\
\text { integration points }\end{array}$ & Min (PSI) & $\operatorname{Max}(\mathrm{PSI})$ & $\begin{array}{l}\text { Average } \\
\text { (PSI) }\end{array}$ \\
\hline $\begin{array}{l}\text { Anterior Fontanelle and } \\
\text { Frontal Suture }\end{array}$ & 273 & 0.04 & 0.48 & 0.21 \\
\hline Sagittal Suture & 143 & 0.01 & 0.25 & 0.13 \\
\hline Posterior Fontanelle & 146 & 0.15 & 0.59 & 0.33 \\
\hline Left Corronal Suture & 80 & 0.29 & 1.20 & 0.77 \\
\hline Right Corronal Suture & 102 & 0.04 & 2.40 & 0.37 \\
\hline Left Sphenoid Fontanelle & 64 & 0.08 & 7.62 & 1.59 \\
\hline Right Sphenoid Fontanelle & 60 & 0.03 & 1.72 & 0.31 \\
\hline Left Squamosal Suture & 51 & 0.60 & 1.70 & 1.03 \\
\hline Right Squamosal Suture & 55 & 0.24 & 0.64 & 0.43 \\
\hline Left Mastoid Fontanelle & 87 & 0.33 & 0.80 & 0.57 \\
\hline Right Mastoid Fontanelle & 61 & 0.14 & 0.47 & 0.30 \\
\hline Left Lamboidal Suture & 90 & 0.26 & 0.71 & 0.48 \\
\hline Right Lamboidal Suture & 103 & 0.07 & 0.65 & 0.43 \\
\hline
\end{tabular}

Table 5- Stress Value Results for Model 3: Number of elements, minimum, maximum, and average Von Mises stress values off all 13 different suture sections for the PP patient in supine position wearing an orthotic helmet. 
The range of stress values obtained from the 13 different suture regions of models 1,2 , and 3 as well as the average stress value for each population are demonstrated by Figure 26 .

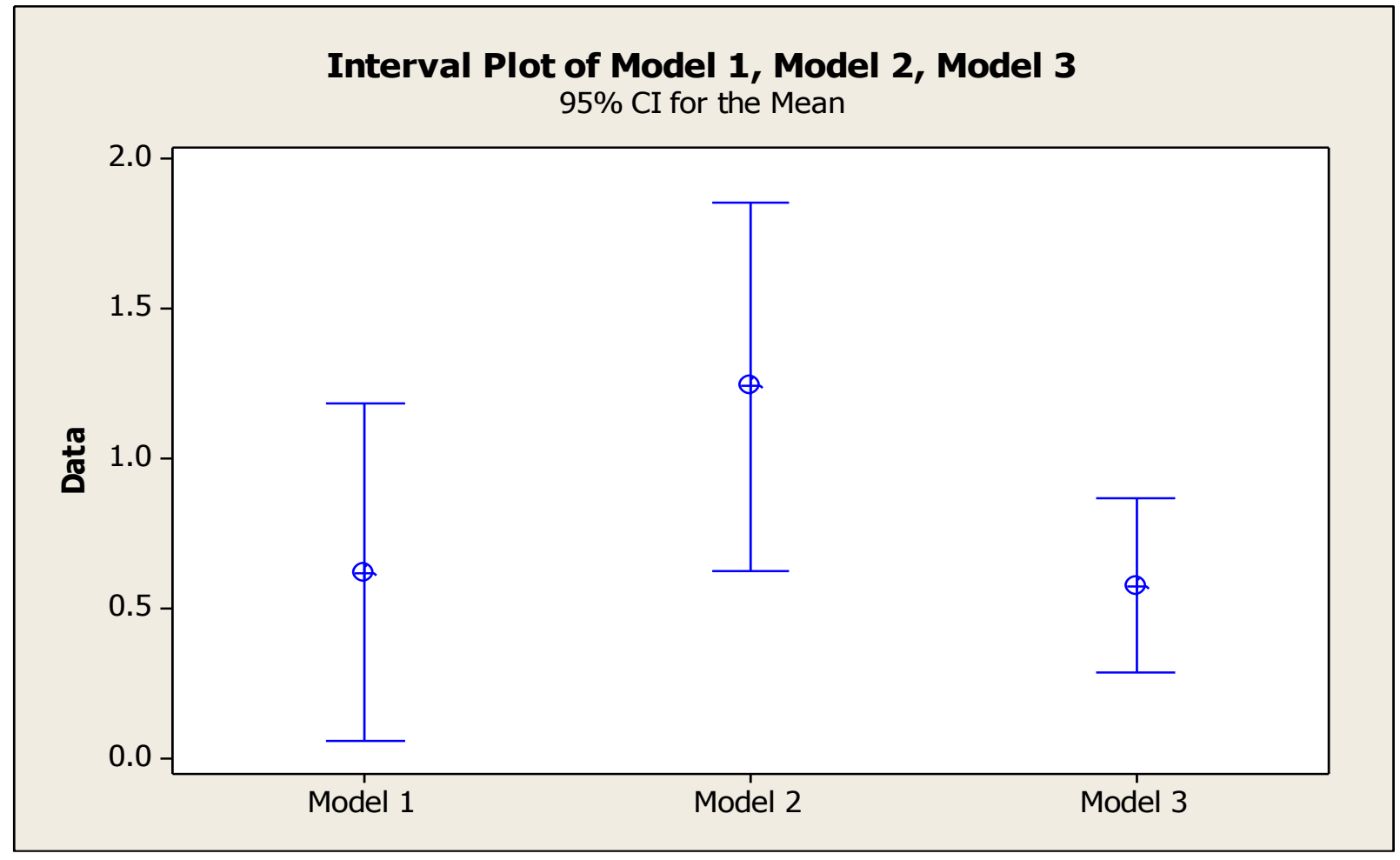

Figure 26- Interval plots of the three populations of average Von Mises values for models 1, 2 and 3.

Table 6 summarizes the descriptive statistics for each population.

\section{Descriptive Statistics: Model 1, Model 2, Model 3}

$\begin{array}{lrrrrrrrrrr}\text { Variable } & N & N^{*} & \text { Mean } & \text { SE Mean } & \text { StDev } & \text { Minimum } & \text { Q1 } & \text { Median } & \text { Q3 } & \text { Maximum } \\ \text { Model 1 } & 13 & 0 & 0.621 & 0.259 & 0.932 & 0.004 & 0.026 & 0.425 & 0.638 & 3.318 \\ \text { Model 2 } & 13 & 0 & 1.239 & 0.282 & 1.018 & 0.256 & 0.386 & 0.927 & 2.113 & 3.465 \\ \text { Model 3 } & 13 & 0 & 0.577 & 0.132 & 0.477 & 0.129 & 0.302 & 0.431 & 0.671 & 1.590\end{array}$

Table 6-Descriptive Satistics of the three populations of average Von Mises stress values.

Figure 27 compares the three different average Von Mises Stress values of each individual suture segment for models 1, 2 and 3 . Bars with blue, orange, and grey colors represent models 1,2 , and 3 respectively. 


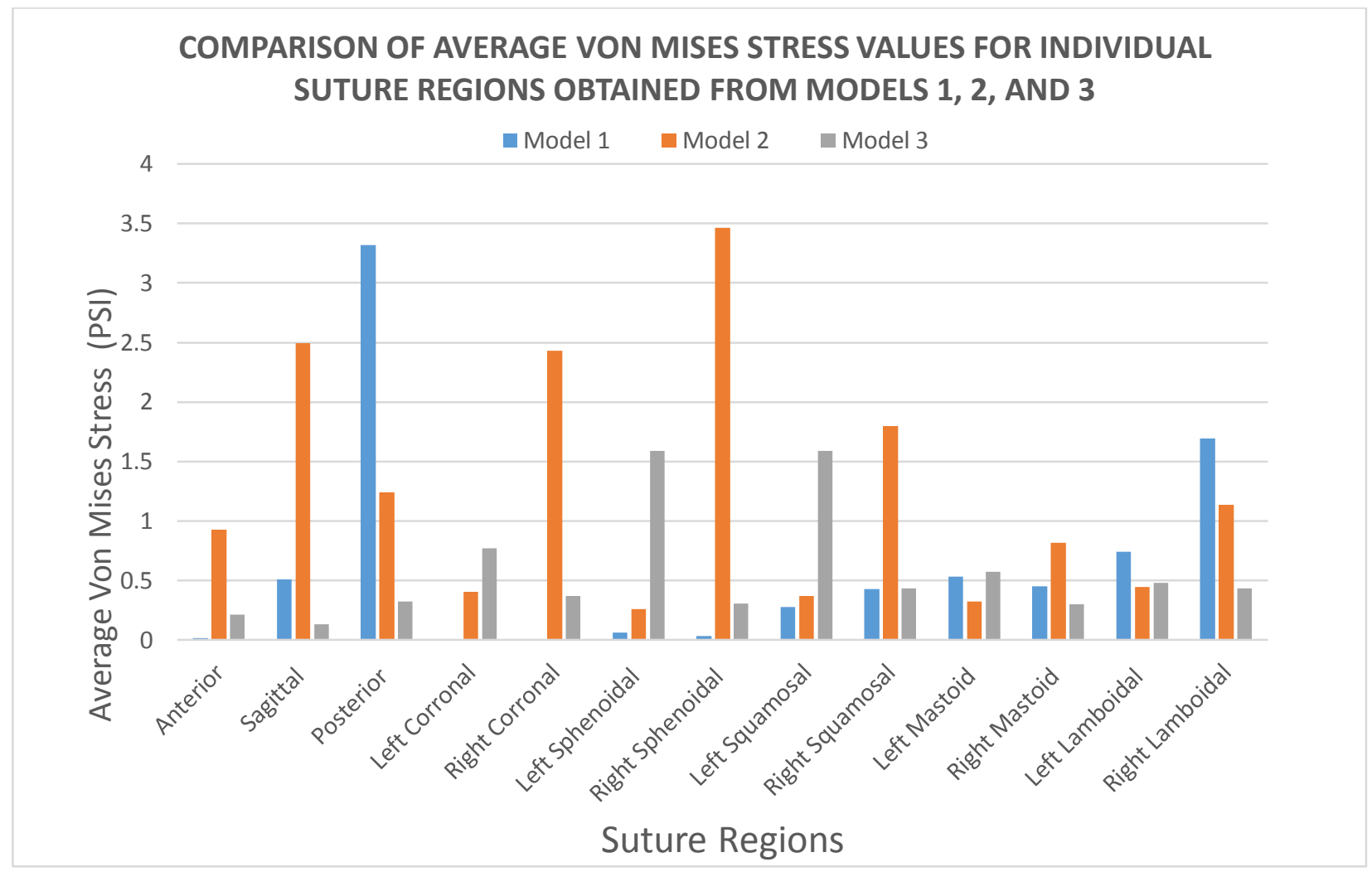

Figure 27- Side-by-side comparison of average Von Mises stress values for all 13 individual suture regions of models 1,2 , and 3 .

Model Validation

In order to assess the validity of the values resulted from the models, a simplified problem was designed and solved for each of the three models. The purpose of solving these problem was to find and compare the order of magnitude of the theoretical pressure exerted on the pillow or helmet due to the weight of the baby's head to the pressure values obtained from the FE models. Pressure was specifically chosen as the target quantity for validation since it only contains the perpendicular forces to the surfaces which makes calculations simpler to perform.

Using the Query Tool inside the Abaqus environment, pressure values corresponding to elements of the surface area of the pillow directly in contact with the baby's head in Model 1 (Figure 28) were probed from 364 integration points from the surface elements. The average 
pressure value of these elements was found to be $3.6 \mathrm{lbf} / \mathrm{in}^{2}$. This area measures to be about 1.1 square inches area and is represented by the red region on the figure below:

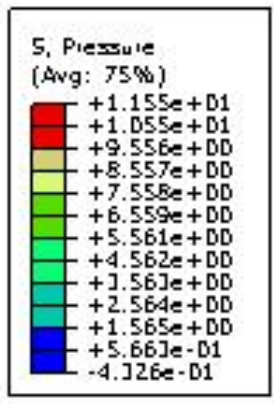

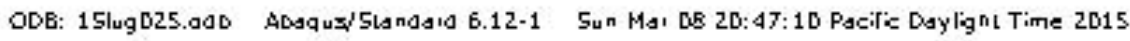

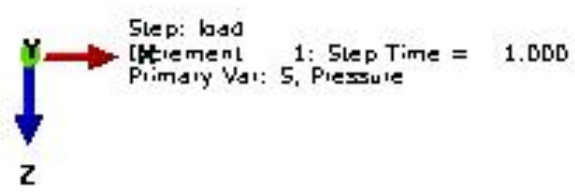

Figure 28-Color contour plot of pressure distribution over the surface of the pillow in Model 1. Pressure values are in PSI. 
Assuming that the weight of the baby's head is distributed uniformly around this area, the theoretical average pressure value over the pillow can be calculated as follows:

$$
\begin{gathered}
\text { Average Pressure over Pillow }=\frac{\text { Weight of the Baby's Head }}{\text { Contact Area }} \\
\text { Average Pressure over Pillow }=\frac{4.1 \mathrm{lbf}}{1.1 \mathrm{in}^{2}} \\
\text { Average Pressure over Pillow }=3.7 \frac{\mathrm{lbf}}{\mathrm{in}^{2}}
\end{gathered}
$$

The difference between the calculated value and the value obtained from the model can then be calculated as follows:

$$
\begin{gathered}
\% \text { Difference }=\left|\frac{\text { Theoretical Value-Actual Value }}{\text { Theoretical Value }}\right| \times 100 \\
\% \text { Difference }=\left|\frac{3.7-3.6}{3.7}\right| \times 100 \\
\% \text { Difference }=2.7 \%
\end{gathered}
$$

Similarly, the theoretical pressure values on the pillow and helmet for models 2 and 3 respectively were calculated and compared to the values obtained from the FE models with the same assumptions. Table 9 summarizes the results from this analysis. Figures 30 and 31 show the pressure distributions over the surface of the pillow and the interior surface of the helmet for models 2 and 3 respectively. 


\begin{tabular}{|c|c|c|c|c|c|}
$\begin{array}{c}\text { Model } \\
\text { Number }\end{array}$ & $\begin{array}{c}\text { Surface } \\
\text { Contact } \\
\text { Area }\left(\mathrm{in}^{2}\right)\end{array}$ & $\begin{array}{c}\text { Number of int. } \\
\text { pts. on the } \\
\text { contact Surface }\end{array}$ & $\begin{array}{c}\text { Theoretical } \\
\text { Pressure } \\
\text { Value }\left(\mathbf{l b f} / \mathrm{in}^{2}\right)\end{array}$ & $\begin{array}{c}\text { Pressure Value } \\
\text { from FE Model } \\
\left(\mathbf{I b f} / \mathrm{in}^{2}\right)\end{array}$ & $\begin{array}{c}\text { Percentage } \\
\text { Difference }\end{array}$ \\
\hline Model 1 & 1.1 & 364 & 3.7 & 3.6 & 2.7 \\
\hline Model 2 & .8 & 224 & 5.3 & 5.8 & 9.4 \\
\hline Model 3 & 127 & 22124 & .03 & .0003 & 99.0 \\
\hline
\end{tabular}

Table 7- Comparing the calculated pressure values.
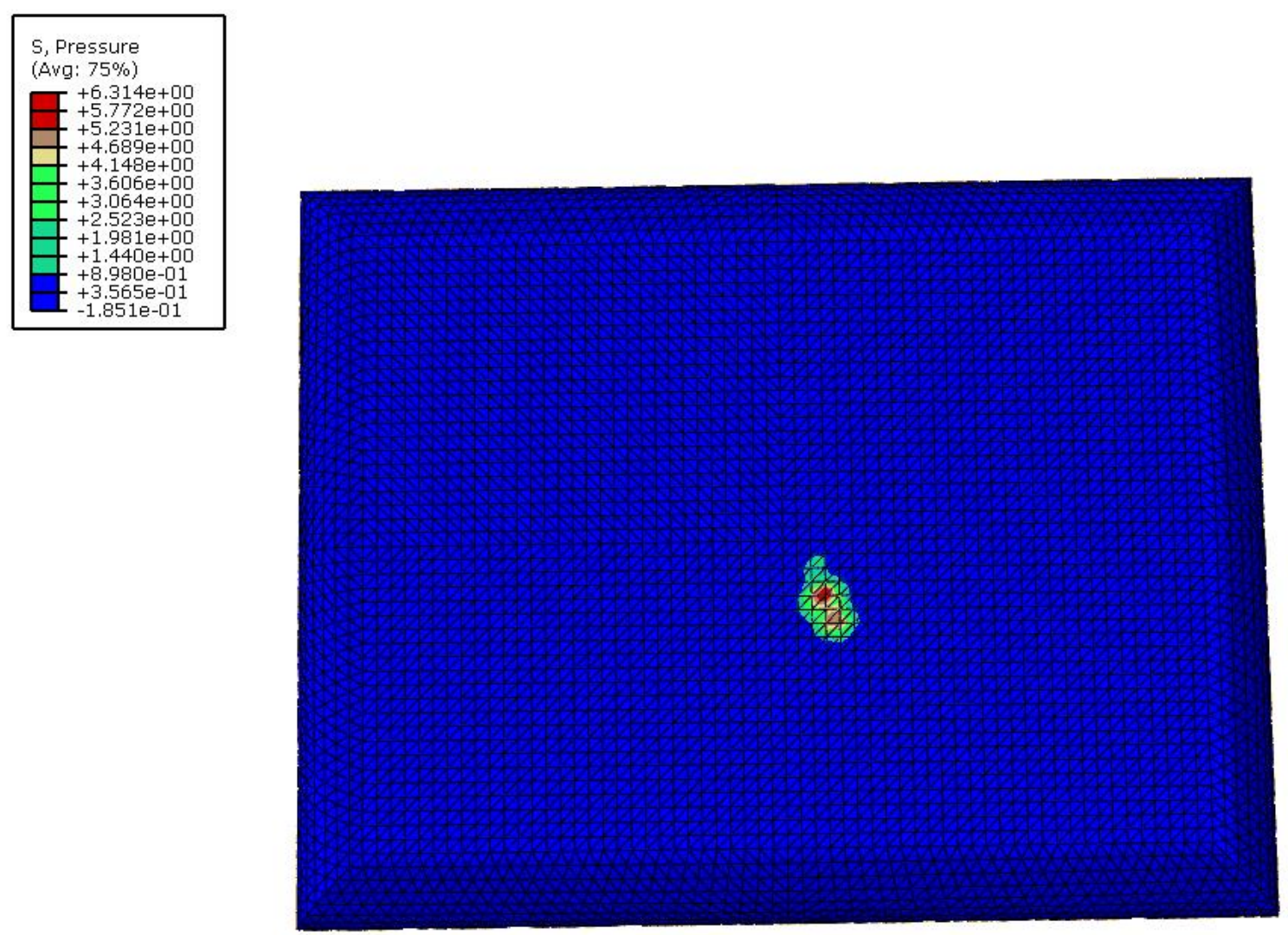

ODB: Job-12.odb Abaqus/Standard 6.12-1 Tue Mar 03 21:32:26 Pacific Standard Time 2015

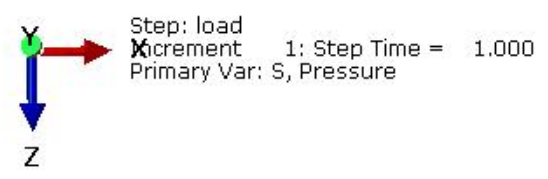

Figure 29- Color contour plot of pressure distribution over the surface of the pillow in Model 2. Pressure values are in PSI. 

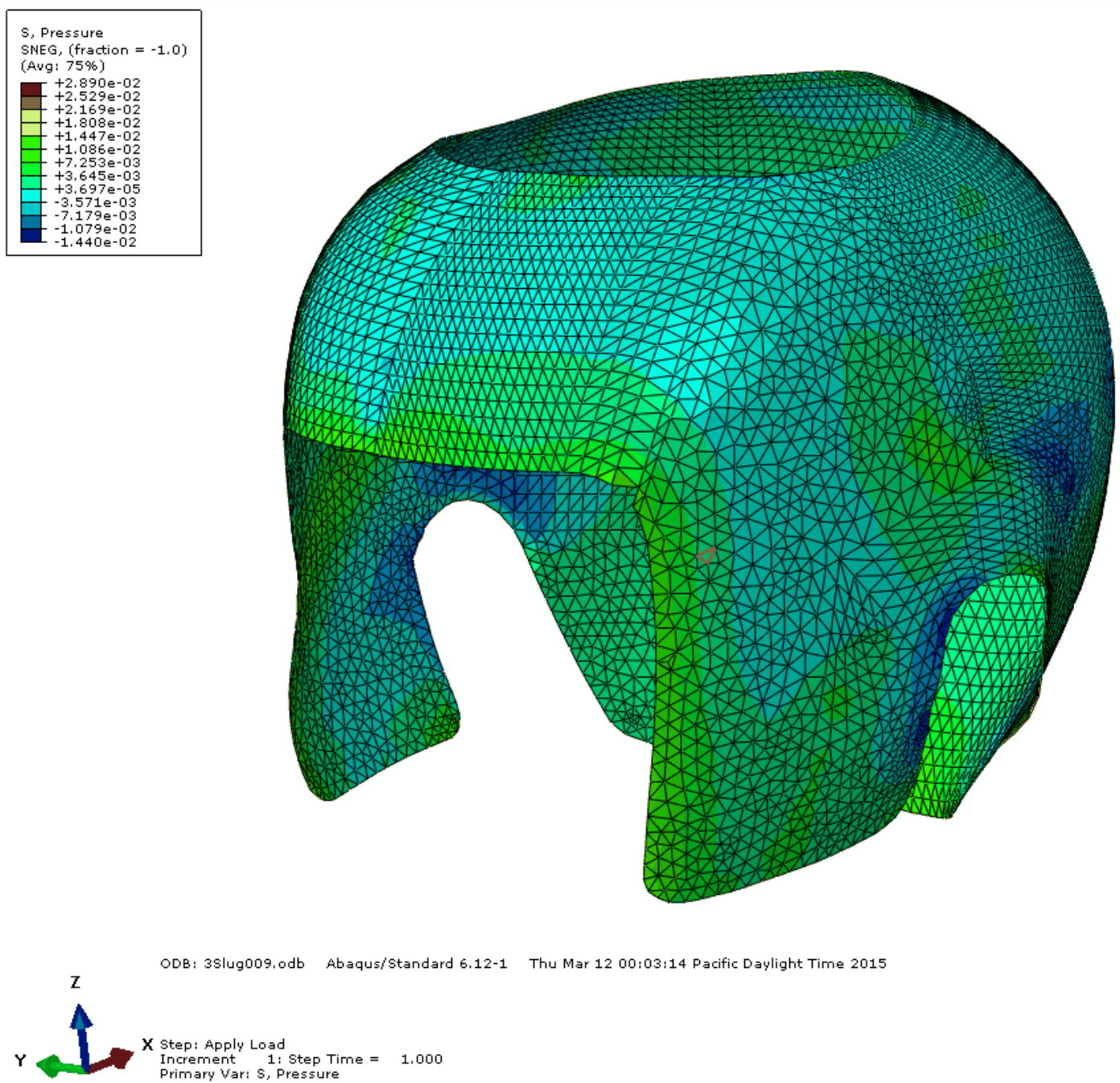

Figure 30- Color contour plot of pressure distribution over interior surface of the helmet in Model 3. Pressure values are in PSI.

As it may be noted from Table 9, there was only a $2.7 \%$ and $9.4 \%$ difference between the theoretical value and the FE model values for models 1 and 2 respectively due to the simplifying assumptions that were made for the calculated theoretical values. The differences are insignificant and suggest that models 1 and 2 resulted in fairly valid outputs.

The value obtained from model 3 is $99 \%$ lower than the calculated value. Looking at Figure 31, it may be observed that our model generated a pressure distribution inside the 
helmet with areas of negative and positive values (represented by blue and green colors respectively) due to a surface distortion caused by the friction-less contact of the baby's head with the relatively soft shell elements inside the helmet. On the other hand, the pressure values were assumed to be all in the negative direction in our calculation. The positive and negative values would then cancel each other out when calculating an average pressure and result in a significant decrease in the value compared to the theoretical value. Therefore, the complex geometries, over-simplifying assumptions, and the unrealistic distortion of the foam layer inside the helmet in model 3 limit our ability in validating this model.

\section{Discussion}

Based on the data summarized in Tables 3 and 4, the highest amount of stress is found to be around the suture regions that were closest to the point of contact of the baby's head with the pillow for Models 1 and 2 (posterior fontanelle in model 1, right sphenoidal fontanelle, the right coronal, and sagittal sutures in model 2. The data in tables 3,4 , and 5 suggest that sleeping with the cranial helmet would decrease the average Von Mises stress within the posterior fontanelle by $69 \%$ compared to the healthy baby sleeping in supine position and $21 \%$ compared to the deformed head sleeping on the flat surface of the head.

Looking at the size of interval plots created using the three sets of 13 individual average Von Mises stress values for the three models (Figure 26), it may be noted that the data sets for models 1 and 2 resulted in a larger intervals plots compared to model 3 . Similarly, the standard deviations, as shown in Table 5, are higher for models 1 and 2 in comparison with model 3. This effect clearly supports the belief that wearing the helmet results in a more uniformly distributed stress all around different suture regions of the baby's skull which results in a smaller standard deviation and interval plot. 
By comparing the results obtained from the models to the data gathered during clinical trials, it may be found whether or not the results obtained from the models are in accordance with the outcome of cranial molding in clinical use, and try to find a correlation between Von Mises stress distribution within critical sutures and effectiveness of the treatment.

In a study performed by Schweitzer et al., 20 female and 31 male Caucasian infants diagnosed with PP with ages ranging from 3.07 to 7.17 months were subjects of an experiment that evaluated the effectiveness of cranial molding on correcting the head geometry of PP patients (32). The subjects of this study were prospectively recruited from June 2010 to January 2012. Three dimensional stereophotogrammetry was performed as data set for creating the cranial helmet for each individual patient as well as for basic morphometric analysis. The control group consisted of 37 Caucasian infants from 4.93-7.10 months without apparent asymmetry which provided morphometric parameters of what is considered a "normal" head shape and growth. Figure 31 demonstrates how the axes and planes were orientated for taking the three dimensional measurements using the STL files obtained from the patients in the study (32).
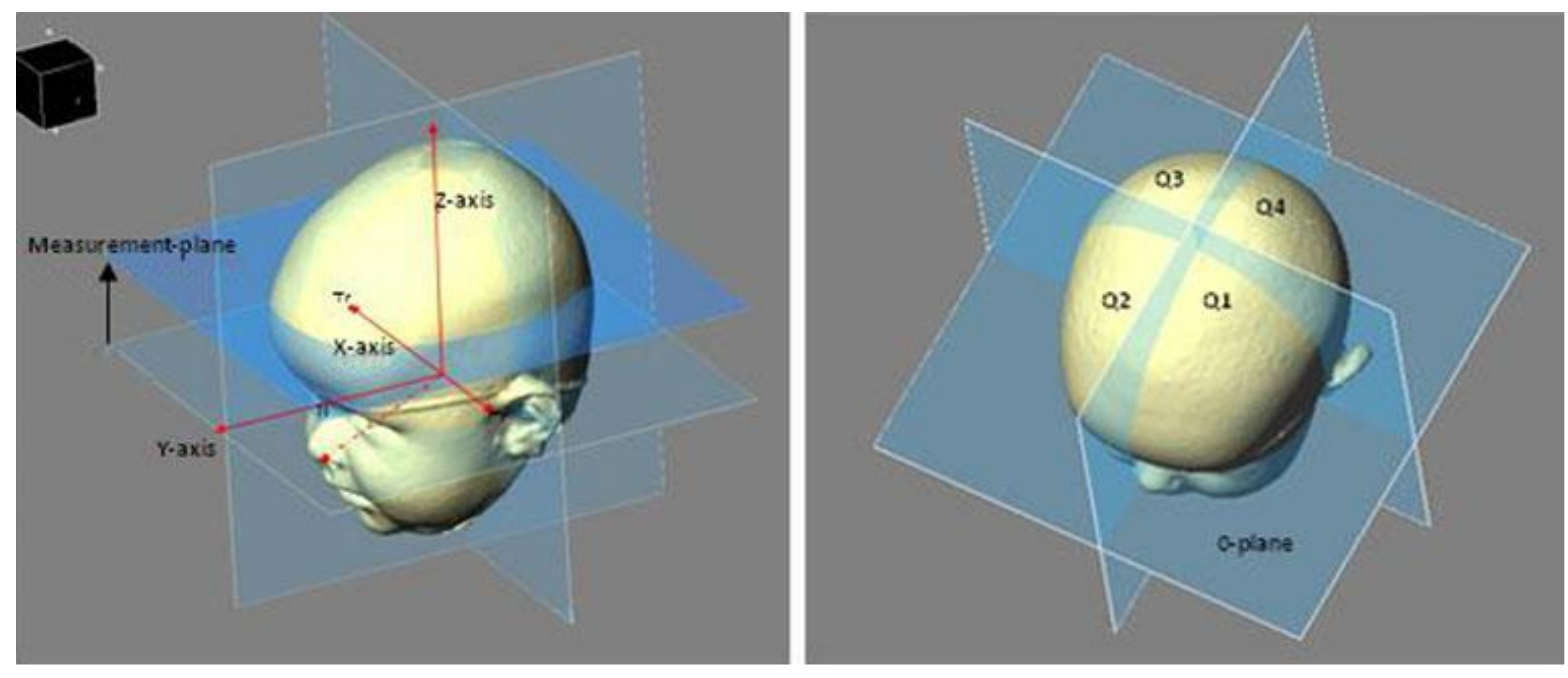

Figure 31- Orientation of three dimensional measurements. Setting up $x$-axis, $y$-axis, $z$-axis, and 0-plane: Measurement plane is the 0-plane shifted parallel to the level of maximum posterior curvature in the occiput (left). Demonstration of volume quadrants from 0-plane (right) (31). 
The growth related parameters and morphometric measurements are described in Table 8.

\begin{tabular}{|c|c|c|}
\hline \multicolumn{3}{|l|}{ Growth related } \\
\hline Circumference & $\mathrm{cm}$ & Head circumference on measurement plane \\
\hline Width & $\mathrm{cm}$ & Maximum skull width on measurement plane \\
\hline \multirow[t]{2}{*}{ CI index } & $\%$ & Cranial index \\
\hline & & Ratio of maximum width to maximum length on measurement plane times 100 \\
\hline Vertex height & $\mathrm{cm}$ & Maximum of skull height from 0 -plane \\
\hline \multicolumn{3}{|l|}{ Symmetry related } \\
\hline Diagonal difference & $\mathrm{cm}$ & $\begin{array}{l}\text { Comparing longest and shortest oblique parameters in the same angle on } \\
\text { measurement plane }\end{array}$ \\
\hline Ear shift & $\mathrm{cm}$ & Difference of ear offset on 0-plane in sagittal direction \\
\hline \multirow[t]{2}{*}{ ACAI } & $\%$ & $\begin{array}{l}\text { Comparison of both anterior cuboid volumes: } \\
\text { (larger cuboid volume--smaller cuboid volume) } \times 100\end{array}$ \\
\hline & & smaller cuboid volume \\
\hline \multirow[t]{2}{*}{ PCAI } & $\%$ & Comparison of both posterior cuboid volumes: \\
\hline & & (larger cuboid volume - smaller cuboid volume $) \times 100$ \\
\hline
\end{tabular}

Table 8- Growth related parameters and morphometric measurements used in the study performed by Schweitzer e al (32).

The results of the morphometric analysis before and after cranial molding are summarized in Table 9 for the patients and the control group. T1 in this table refers to the time at which the initial morphometric measurements were taken prior to the treatment, and T2 refers to the end of the treatment period after implementation of cranial molding. The decision to terminate the molding therapy was made when a level of cranial symmetry was achieved that was satisfying for the parents. The study was conducted from June 2010 to 2012 with an average of 4.5 months $( \pm 1.5)$ for the duration of each treatment $(\mathrm{T} 1-\mathrm{T} 2)(32)$. 


\begin{tabular}{|l|c|c|c|c|c|}
\hline & Units & $\begin{array}{c}\text { Patients } \\
\text { T1-T2 }\end{array}$ & $\begin{array}{c}\text { Control } \\
\text { T1-T2 }\end{array}$ & $\begin{array}{c}\% \text { Difference } \\
\text { between Control } \\
\text { and Patient Groups } \\
\text { T1-T2 }\end{array}$ & $\begin{array}{c}\% \text { Shift } \\
\text { Towards the } \\
\text { Control } \\
\text { Group T1-T2 }\end{array}$ \\
\hline Growth Related Parameters \\
\hline Circumference & $\mathrm{Cm}$ & $43.28-45.57$ & $43.36-46.06$ & $1.8 \%-1.1 \%$ & $0.7 \%$ \\
\hline Width & $\mathrm{Cm}$ & $12.98-13.32$ & $12.47-13.09$ & $3.9 \%-1.7 \%$ & $2.2 \%$ \\
\hline Length & $\mathrm{Cm}$ & $14.25-15.40$ & $14.85-15.93$ & $4.0 \%-3.3 \%$ & $0.7 \%$ \\
\hline Cl index & $\%$ & $91.19-84.91$ & $84.27-82.29$ & $8.2 \%-3.2 \%$ & $5 \%$ \\
\hline Vertex Height & $\mathrm{Cm}$ & $11.18-12.02$ & $10.97-11.60$ & $1.9 \%-3.6$ & $-1.7 \%$ \\
\hline $\begin{array}{l}\text { Symmetry-related Parameters } \\
\text { Diagonal } \\
\text { Difference }\end{array}$ & $\mathrm{Cm}$ & $1.11-0.52$ & $0.35-0.33$ & $217.1 \%-57.57 \%$ & $159.5 \%$ \\
\hline Ear Shift & $\mathrm{Cm}$ & $0.50-0.37$ & $0.21-0.19$ & $138.1 \%-94.7 \%$ & $43.3 \%$ \\
\hline ACAl & $\%$ & $5.17-4.43$ & $3.08-2.78$ & $67.86 \%-59.35 \%$ & $8.5 \%$ \\
\hline PCAl & $\%$ & $20.99-8.07$ & $6.20-5.92$ & $238.5 \%-36.3 \%$ & $202.2 \%$ \\
\hline
\end{tabular}

Table 9- The results of the morphometric analysis at T1 and T2 (32).

As it may be noted from the values in Table 5, the symmetry-related parameters that had the most significant shift towards the control group from T1 to T2 were Diagonal Difference and PCAI. Among all parameters in Table 9, these two values are also the best representation of the effectiveness of cranial molding since they directly correlate to the changes in the asymmetry of the back of the baby's head in posterior, lamboidal, and sagittal suture regions. The stress values of these sutures obtained from models 1 and 3 can therefore be compared to the Diagonal Difference and PCAI values obtained from morphometric measurements at $\mathrm{T} 1$ and T2 during the clinical trials to better understand how the Von Mises stress distributions before and after wearing the helmet (models 1 and 3) relate to the baby's skull growth from T1 and T2. 
A side-to-side comparison of the average Von Mises stress values for each skull suture within models 1 and 3 is shown on Figure 27. It may be noted that the values for the left lamboidal, right lamboidal, sagittal, and posterior sutures have decreased by $35.1 \%, 74.5 \%$, $74.5 \%$, and $90.0 \%$ respectively from Model 1 to Model 3 due to the effects of wearing the cranial molding helmet during sleep. A summary of the stress values and the percentage of reduction of stress (after wearing the helmet) for each of the suture segments are shown in Table 10.

\begin{tabular}{|l|c|c|c|}
\hline & $\begin{array}{l}\text { Average Von Mises Stress } \\
\text { Value (PSI) in Model 1 }\end{array}$ & $\begin{array}{l}\text { Average Von Mises Stress } \\
\text { Value (PSI) in Model 3 }\end{array}$ & $\begin{array}{l}\text { \% } \\
\text { Difference }\end{array}$ \\
\hline Left Lamboidal & .74 & .48 & 35.1 \\
\hline Right Lamboidal & 1.69 & .43 & 74.5 \\
\hline Sagittal & .51 & .13 & 74.5 \\
\hline Posterior & 3.32 & .33 & 90.0 \\
\hline
\end{tabular}

Table 10-Comparing average Von Mises Stress Values of Left Lamboidal, Right Lamboidal, Sagittal, and Posterior sutures obtained from models 1 and 3.

The reduction of stress (as shown in Table 10) is in accordance with the outcome of the clinical study discussed earlier. Wearing the cranial helmet during sleep relieves the pressure from one area at the back of the baby's head and distributes it all around the baby's skull which assists the natural symmetric skull growth of all regions. The results of the two studies suggest that a $35 \%$ to $90 \%$ reduction of stress from the back of the head of a PP patient during sleep may lead to an average of $160 \%$ correction in the Diagonal Difference and a $202 \%$ correction in PCAI during 4.5 months of therapy.

\section{Limitations}

Limitations of the models are mainly associated with the simplifying assumptions and geometries in generating and validating the FE models. A few of such limitations are addressed in this section. 
The laser scanned files of the baby's head were obtained in STL format and only defined the surface contour of the baby's head. Therefore, in order to mesh the helmet and the head contours in the models, shell elements with a uniform thickness were used. In reality, the thickness of human skull and its sutures are non-uniform and variable. The results of the simulations would have been more accurate if the mesh was generated over a 3D CT scanned file instead of laser-scanned surface contour.

Similarly, the suture lines were manually traced over the STL files to represent the approximate geometry of the sutures and neglected the effects of the micro-level complexities of the geometry. In reality, the boarder-lines between sutures and bones within an infant's skull has many small details such as sharper angles and distorted lines (vs. smooth lines of the simplified models in this study) which can create several stress concentrations and skewed results. Such small details in the geometry of the sutures are also variable from one person to another person and depends on genetic and environmental factors associated with each individual. Therefore, it's impossible to create one model that accurately generates results that resembles all individuals.

The effects of cranial pressure, atmospheric pressure, muscle forces, soft tissues, anisotropic behavior of the materials, and friction between materials were neglected in the models; all of which could have had a significant effect on the results obtained from the models.

Also, the boundary conditions either eliminated or neglected the effects of side-to-side rotation of the head around the neck joints in order to create models that converge to a solution. A more precise model would ideally incorporate all loading conditions and boundary conditions that were excluded from the models in this study.

Furthermore, validation of the models was done by comparing the results of calculations with many simplifying assumptions. As a result of making such simplifying 
assumptions in the calculations, the values obtained from the calculations may have been significantly different from an ideal case. However, without having quantitative experimental or clinical data to compare with the results of the models, solving a simplified mathematical problem might arguably be the best approach for validation of the models.

\section{Future Directions}

Future studies need to focus on over-coming the limitations discussed and develop similar models with less simplifying assumptions and include more complexities and variables for more accurate results.

Clinical data and studies surrounding implementation of medical devices for treatment of positional plagiocephaly lack a focus on methods that quantify distribution of stress for evaluating the effectiveness of the devices. Future studies could focus on fulfilling this need by coming up with methods using pressure sensors and force gauges to gather experimental data that may be used to validate computational models or compare the efficacy of devices to each other.

The methods implemented in this study and the results obtained from the models may be used as a basis for future evaluation and design of engineered solutions for treatment and prevention of PP in new-born babies. For example, a similar model could be generated for a baby sleeping in supine position on a bedding pillow to study and compare the resulting stress distributions within the sutures to the models obtained in this study. Then the geometry and material of the corrective device could be modified accordingly for an optimized uniform stress distribution.

Furthermore, the effects of different loading and boundary conditions on bone remodeling of different regions of the baby's skull can be assessed by combining similar methods used in this study with a bone remodeling algorithm developed by Dr. Scott Hazelwood et al. (33). 
This would help to come up with engineered solutions that not only focus on the efficacy of the device on correcting PP, but also optimize and control the response of bone tissues to stress within the patient's skull.

\section{Conclusions}

There is a considerable market for corrective methods and devices that target treatment of PP. Bedding pillows, cranial helmets, 3D laser scanners, and CAD software packages are examples of technologies engineered for use at orthopedic clinics for treatment of PP. Most of the publications surrounding the use of these devices and methods either compare the effectiveness of each method or device in correcting the head shape of the baby within a specific period of time, or assess finding the shortcomings and side effects of using each method. These studies often lack a focus on quantifying the magnitude and distribution of the stress that either cause or correct head deformations in new born babies for optimizing the efficacy of the engineered products.

In this study, three separate FE models were developed and validated that outputted the magnitudes of Von Mises stresses at different regions of an infant's skull sutures in three different scenarios: a baby without PP sleeping on a pillow in supine position, a baby with PP sleeping on the flat side of his head in supine position, and the same baby with PP wearing a helmet and sleeping in supine position. The goal of the study was to assess the efficacy of implementing a cranial helmet for reducing concentrated stress from the back of the subject's head using a quantitative approach.

The results supported that wearing the cranial helmet reduces concentrated stress from the lamboidal, posterior, and sagittal sutures while the subject sleeps in supine position.

In addition, the data obtained from models 1 and 3 were compared to the morphometric measurements collected before and after implementation of cranial molding during a clinical study. The results showed that the regions with most stress reductions in model 3 (compared to 
model 1) were the ones that correlated the most to the morphometric parameters that had the major shift towards the control group at the end of the clinical study, which confirms that the results of the FE models and the clinical evaluation were in accordance with each other. 


\section{BIBLIOGRAPHY}

1. Mortenson, Patricia, Paul Steinbok, and David Smith. "Deformational Plagiocephaly and Orthotic Treatment: Indications and Limitations." Child's Nervous System: 1407-412. Web. 9 Aug. 2014.

2. Dunn, P. M. "Congenital Sternomastoid Torticollis: An Intrauterine Postural Deformity." Archives of Disease in Childhood (1974): 824-25. Web. 9 Aug. 2014.

3. Graham, John M., Mayela Gomez, Andy Halberg, Dawn L. Earl, Jeannie T. Kreutzman, Jinrui Cui, and Xiuqing Guo. "Management of Deformational Plagiocephaly: Repositioning versus Orthotic Therapy." The Journal of Pediatrics: 258-62. Web. 9 Aug. 2014.

4. Gump, William C., lan S. Mutchnick, and Thomas M. Moriarty. "Complications Associated with Molding Helmet Therapy for Positional Plagiocephaly: A Review." Neurosurgical Focus 35 (2013): E3. Web. 9 Aug. 2014.

5. Lipira, A. B., S. Gordon, T. A. Darvann, N. V. Hermann, A. E. Van Pelt, S. D. Naidoo, D. Govier, and A. A. Kane. "Helmet Versus Active Repositioning for Plagiocephaly: A Three-Dimensional Analysis." Pediatrics(2010): E936-945. Web. 9 Aug. 2014.

6. Cummings, Carl. "Positional Plagiocephaly." Paediatr Child Health (2011): 493-94. Web. 8 Aug. 2014.

7. Block, Stan L. "'Skull-Duggery' and the Management of Positional Plagiocephaly." Pediatric Annals (2012): 497-501. Web. 9 Aug. 2014.

8. Bialocerkowski, Andrea E, Sharon L Vladusic, and Choong Wei Ng. "Prevalence, Risk Factors, And Natural History Of Positional Plagiocephaly: A Systematic Review." Developmental Medicine \& Child Neurology(2008): 577-86. Web. 9 Aug. 2014.

9. Dunn PM. Congenital sternomastoid torticollis: an intrauterine postural deformity. Arch Dis Child 1974;49:824-5.

10. Shamji, Mohammad F., Elana C. Fric-SHamji, Praneal Mechant, and Michael Vassilyadi. "Cosmetic and Cognitive Outcomes of Positional Plagiocephaly Treatment." PubMed (2012): n. pag. NCBI. Web. 29 Apr. 2015. "Plagiocephaly Severity Scale." Actionorthosante.ca/english. Web. 20 Jan. 2015. $<$ http://actionorthosante.ca/english/Plagiocephaly-Severity-Scale.php>.

11. Wilbrand, Jan-Falco, Maximilian Seidl, Martina Wilbrand, Philipp Streckbein, Sebastian Böttger, Joern Pons-Kuehnemann, Andreas Hahn, and Hans-Peter Howaldt. "A Prospective Randomized Trial on Preventative Methods for Positional Head Deformity: Physiotherapy versus a Positioning Pillow." The Journal of Pediatrics 162.6: 1216-221.e1. Web. 9 Aug. 2014.

12. Yoo, Han-Su, Dong Kyun Rah, and Yong Oock Kim. "Outcome Analysis of Cranial Molding Therapy in Nonsynostotic Plagiocephaly." Archives of Plastic Surgery: 338. Web. 23 Aug. 2014.

13. Bialocerkowski, Andrea E, Sharon L Vladusic, and Stephanie M Howell. "Conservative Interventions for Positional Plagiocephaly: A Systematic Review." Developmental Medicine \& Child Neurology 47 (2005): 563-70. Web. 9 Aug. 2014.

14. Cheng JCY, Wong MWN, Tang SP, Chen TMK, Shum SLF, Wong EMC. Clinical determinants of the outcome of manual stretching in the treatment of congenital muscular torticollis in infants. J Bone Joint Surg 2001;83-A:679-87.

15. Wilbrand, Jan-Falco, Kerstin Schmidtberg, Uta Bierther, Philipp Streckbein, Joern PonsKuehnemann, Petros Christophis, Andreas Hahn, Heidrun Schaaf, and Hans-Peter Howaldt. "Clinical Classification of Infant Nonsynostotic Cranial Deformity." The Journal of Pediatrics 161.6: 1120-125.e1. Web. 9 Aug. 2014. 
16. Durigneux, J., M. Dinomais, B. Chevalier, L. Marquois, G. Payneau, and X. Deries. "Cervicocephalic Correction Mattress: A New Therapy in Plagiocephaly and

Torticollis." Annals of Physical and Rehabilitation Medicine: E229. Web. 9 Aug. 2014.

17. Coats, Brittany, and Susan S. Margulies. "Material Properties of Human Infant Skull and Suture at High Rates." Journal of Neurotrauma 23.8 (2006): 1222-232. Web. 9 Aug. 2014.

18. Kluba, S., et al., What is the optimal time to start helmet therapy in positional plagiocephaly? Plastic and reconstructive surgery, 2011. 128(2): p. 492-8.

19. "Cranial Molding for Plagiocephaly." Cranial Molding for Plagiocephaly. Web. 24 Sept. 2014.

20. Web. 24 Sept. 2014. <http://www.firsttoserve.com/CatalogOrthotics/Pediatric/Cranial Molding_Helmets/>.

21. Kim, Se Yon, Moon-Sung Park, Jeong-In Yang, and Shin-Young Yim. "Comparison of Helmet Therapy and Counter Positioning for Deformational Plagiocephaly." Annals of Rehabilitation Medicine: 785-95. Web. 9 Aug. 2014.

22. Gump, William C., lan S. Mutchnick, and Thomas M. Moriarty. "Complications Associated with Molding Helmet Therapy for Positional Plagiocephaly: A Review." Neurosurgical Focus: E3. Web. 23 Aug. 2014.

23. "The Story Of My Son's DOC Band." Monicas Mom Musing. Web. 24 Sept. 2014.

24. "Insignia for Hanger Cranial Band." Insignia ${ }^{\mathrm{TM}}$ for Hanger Cranial Band - Hanger Clinic. Web. 12 Apr. 2015. <http://www.hangerclinic.com/bracingsupport/pediatric/plagiocephaly-treatment/Pages/Insignia-for-Hanger-CranialBand.aspx>.

25. Ozcelik, Babur, Alper Ozbay, and Erhan Demirbas. "Influence of Injection Parameters and Mold Materials on Mechanical Properties of ABS in Plastic Injection Molding." International Communications in Heat and Mass Transfer 37 (2010): 1359365. Web. 9 Aug. 2014.

26. Web. 14 Feb. 2015. <http://www.cdc.gov/growthcharts/data/who/grchrt_boys_24lw_100611.pdf>.

27. Huelke, Donald. "An Overview of Anatomical Considerations of Infants and Children in the Adult World of Automobile Safety Design." Association for the Advancement of Automotive Medicine 42 (1998): 93-113. Web. 13 Feb. 2015.

28. "Patent EP1648356B1 - Cranial Orthosis for Preventing Positional Plagiocephaly in Infants." Google Books. Web. 24 Sept. 2014.

29. "Patent US20120296249 - In Situ Molded Orthotic and Method for Its Fabrication." Google Books. Web. 24 Sept. 2014.

30. Rodriguez-Perez, Miguel A, Jorge I Gonzalez-Peña, and Jose A De Saja. "Modifying the Structure and Properties of Polyethylene Foams Using Thermal

Treatments." Polymer International (2009): 620-29. Web. 9 Aug. 2014.

31. Schweitzer, Tilmann, Hartmut Böhm, Christian Linz, Beatrice Jager, Lucia Gerstl, Felix Kunz, Angelika Stellzig-Eisenhauer, Ralf-Ingo Ernestus, Jürgen Krauß, and Philipp Meyer-Marcotty. "Three-dimensional Analysis of Positional Plagiocephaly before and after Molding Helmet Therapy in Comparison to Normal Head Growth." Child's Nervous System (2013): 1155-161. Print. 12 Mar. 2015.

32. Hazelwood, Scott J, R Bruce Martin, Mark M Rashid, and Juan J Rodrigo. "A Mechanistic Model for Internal Bone Remodeling Exhibits Different Dynamic Responses in Disuse and Overload." Journal of Biomechanics (2001): 299-308. Print. 12 Mar. 2015 


\title{
APPENDICES
}

Appendix A: Color Contour Plots of Von Mises Stress Distribution within Suture Segments of Model 1
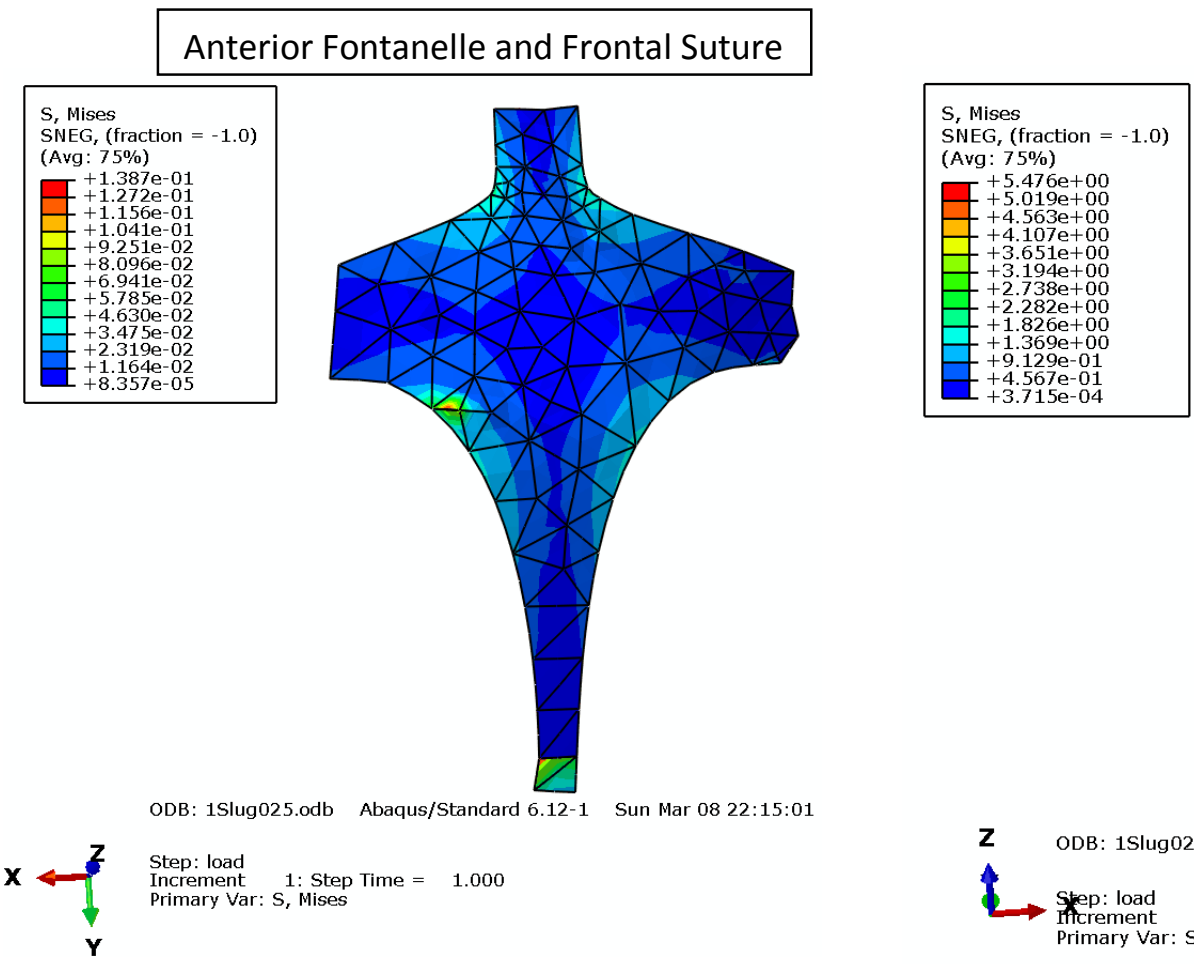

\author{
Sagittal Suture
}

ODB: 1Slug025.odb Abaqus/Standard 6.12-1 Sun Mar 08 22:15:01
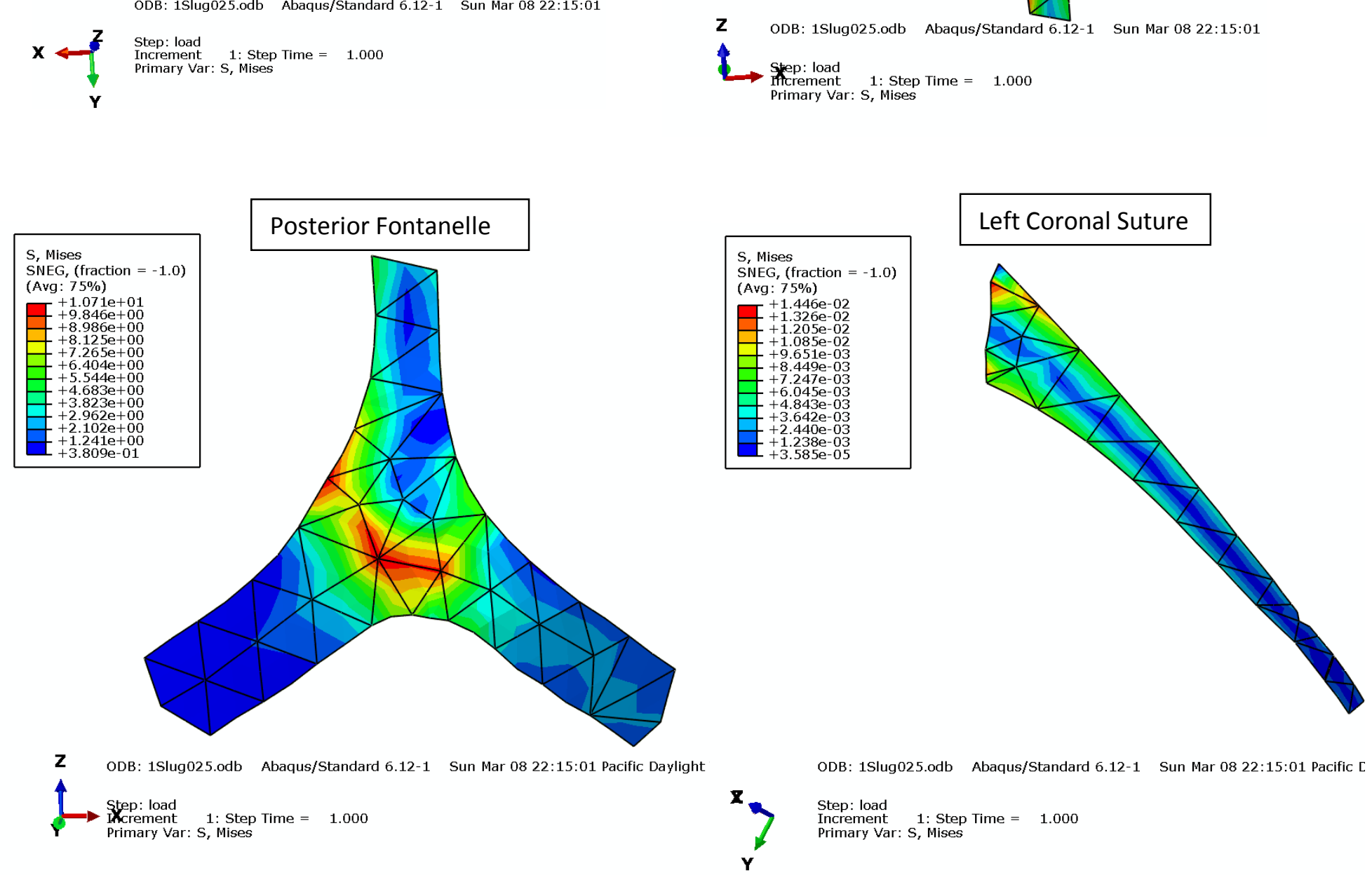


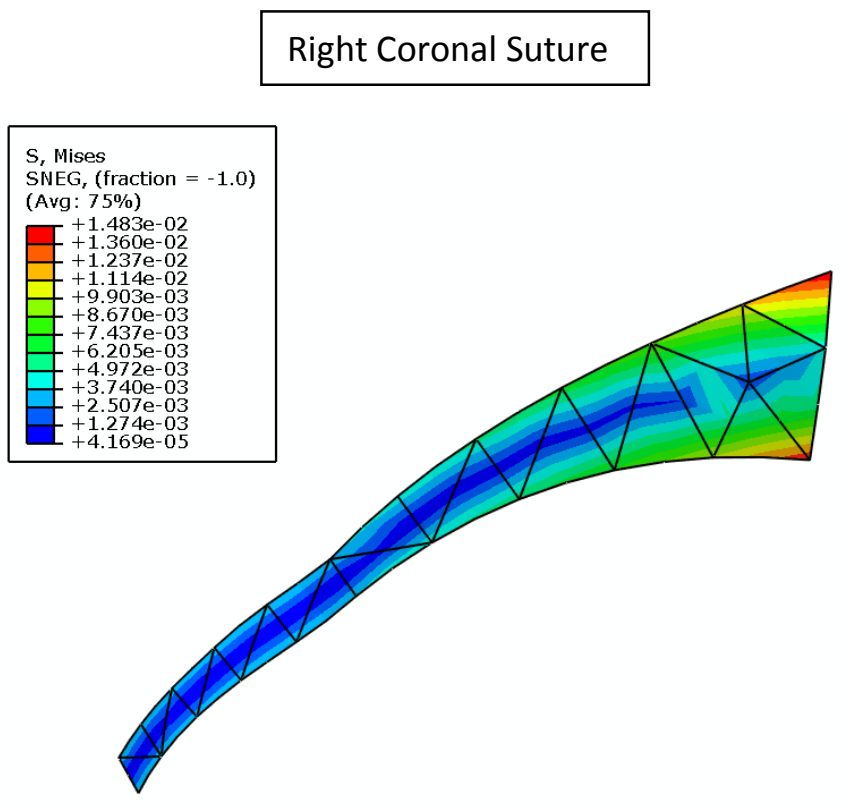

ODB: 1Slug025.odb Abaqus/Standard 6.12-1 Sun Mar 08 22:15:01

$\mathbf{X} \begin{aligned} & \mathbf{S} \\ & \begin{array}{l}\text { Step: load } \\ \text { Increment } \\ \text { Primary Var: S, Mises }\end{array} \\ & \mathbf{Y}\end{aligned}$

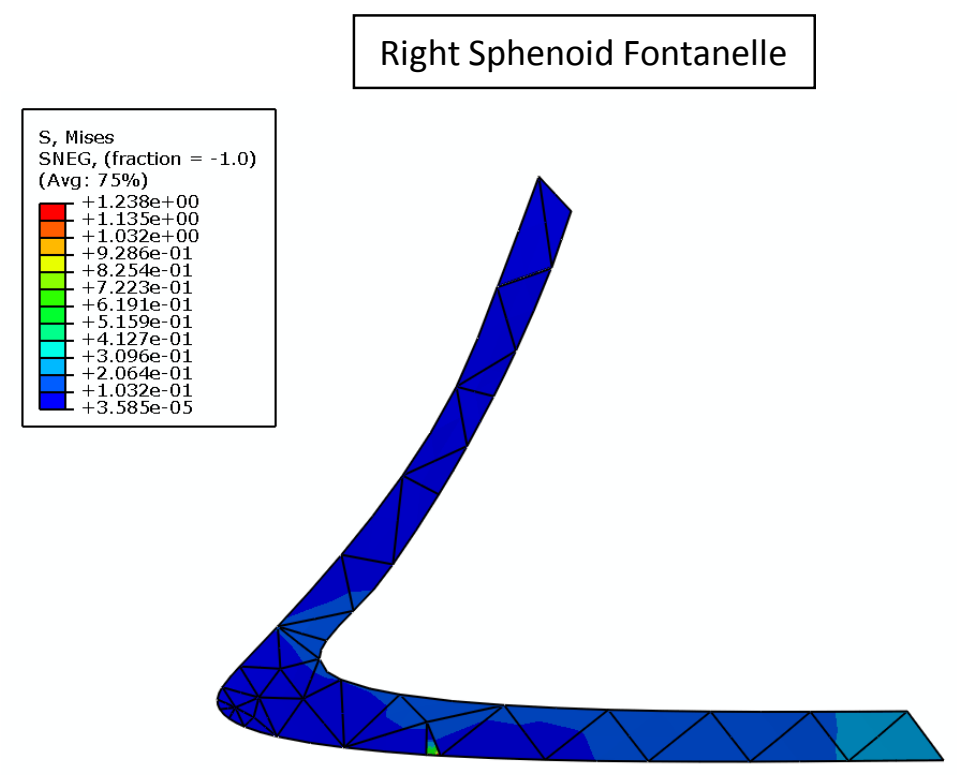

Z ODB: 1Slug025.odb Abaqus/Standard 6.12-1 Sun Mar 08 22:15:01 Pacific Daylight

Step: load

Increment Step Time $=1.000$

Primary Var: S, Mises

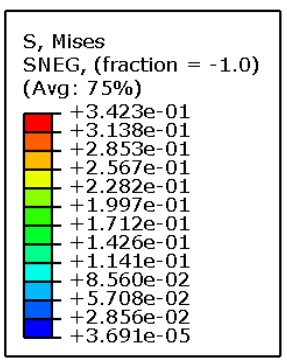

Left Sphenoid Fontanelle
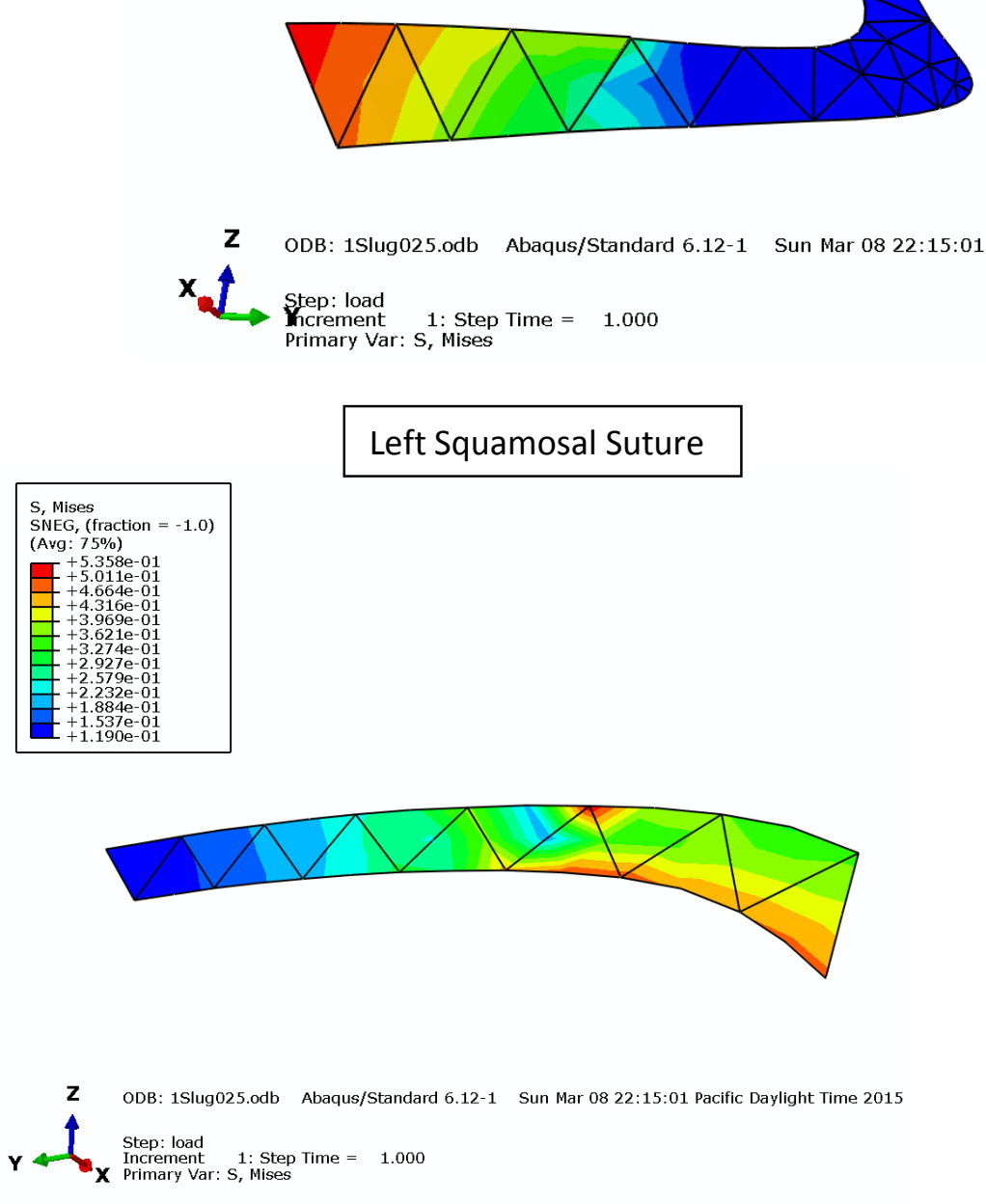

$\mathbf{x + 4}$ 


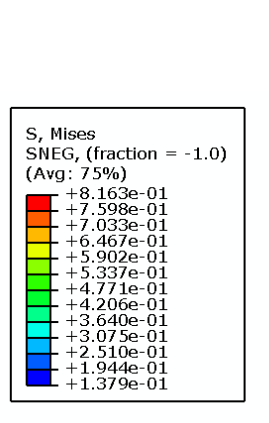

\section{Right Squamosal Suture}

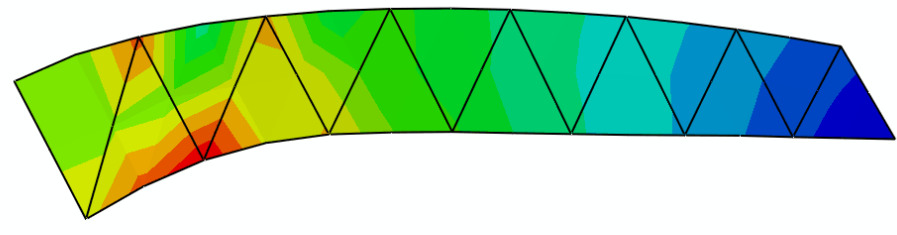

$\mathbf{Z}$ ODB: 1Slug025.odb Abaqus/Standard 6.12-1 Sun Mar 08 22:15:01 Pacific Daylight Time
Step: load
Mrcrement
Primary Var: S, Step Time $=1.000$

Right Mastoid Fontanelle
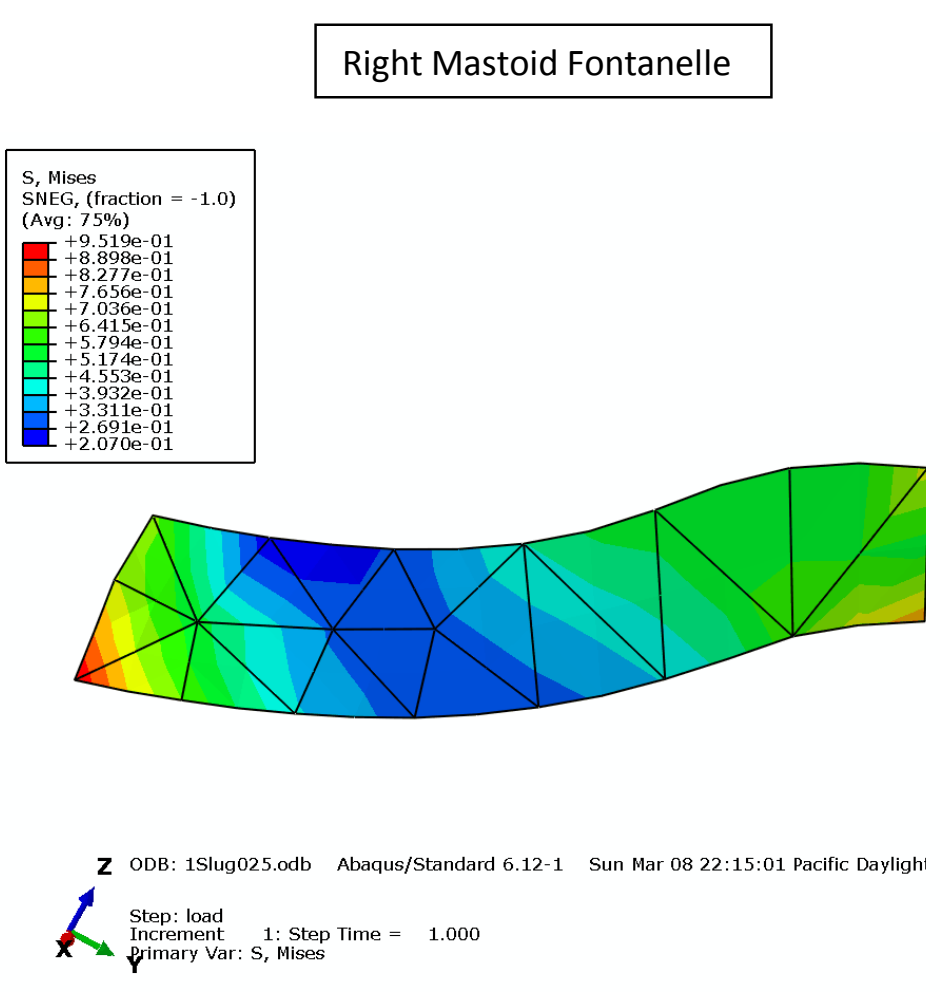

Z ODB: 1Slug025.odb Abaqus/Standard 6.12-1 Sun Mar 08 22:15:01 Pacific Dayligh

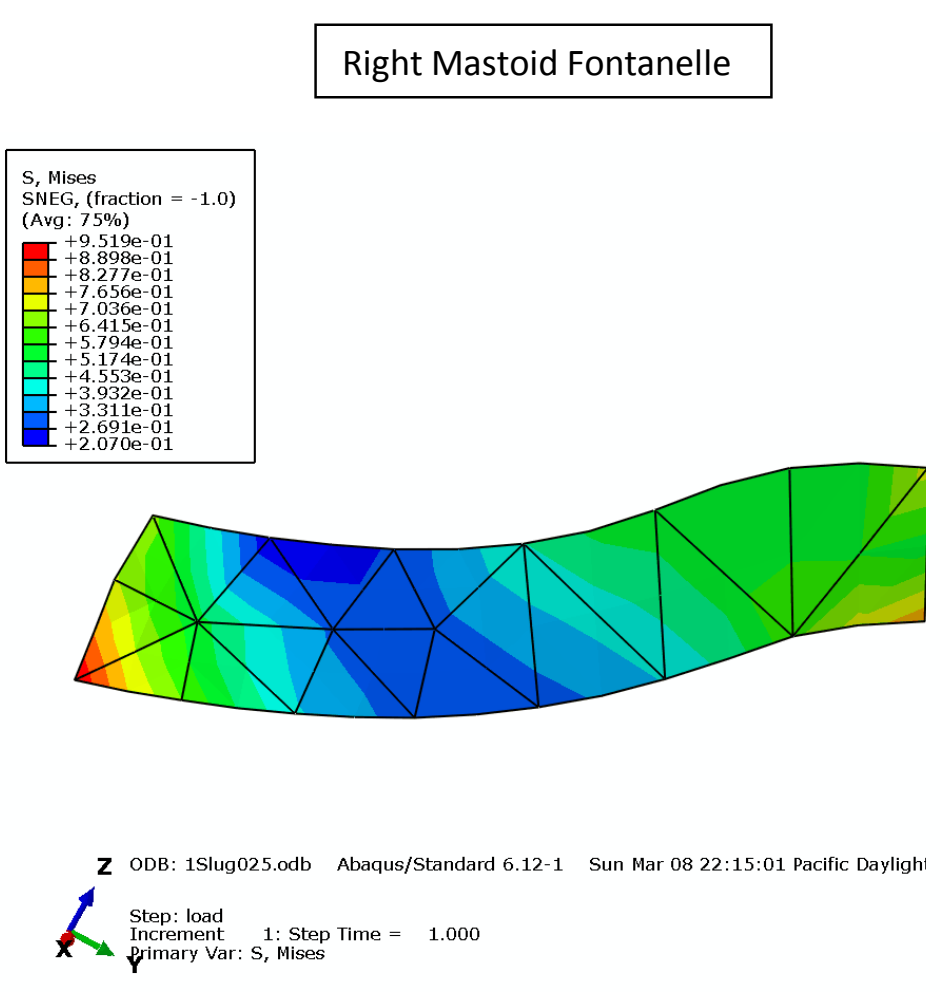

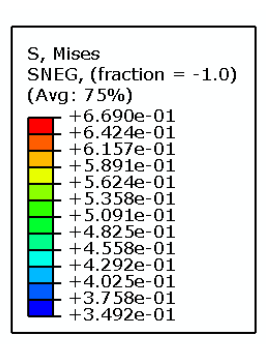

\section{Left Mastoid Fontanelle}

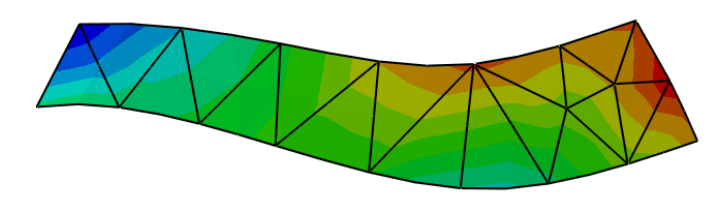

$\mathbf{Z}$ ODB: 1Slug025.odb Abaqus/Standard 6.12-1 Sun Mar 08 22:15:01 Pacific Daylight

Step: load

Increment 1 : Step Time $=1.000$

Primary Var: S, Mises $t_{1}^{z}$
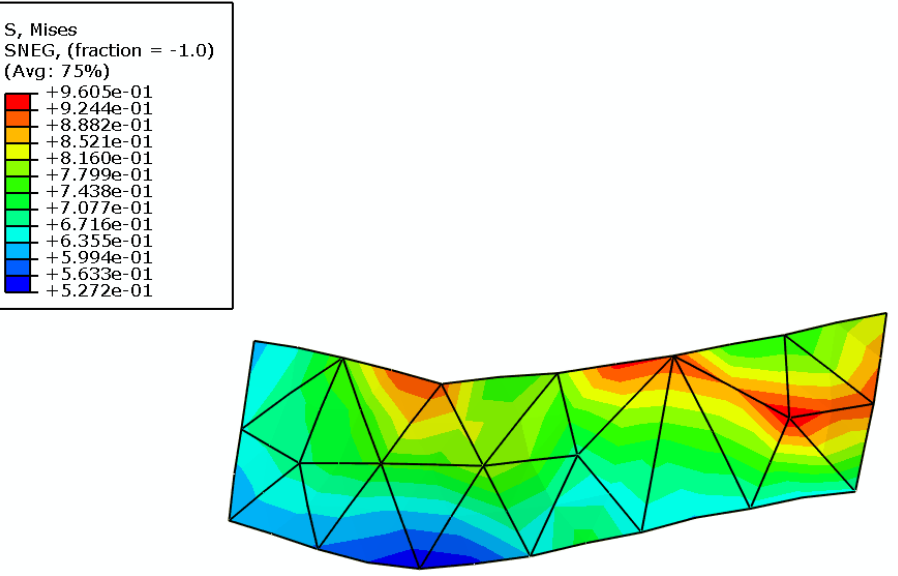


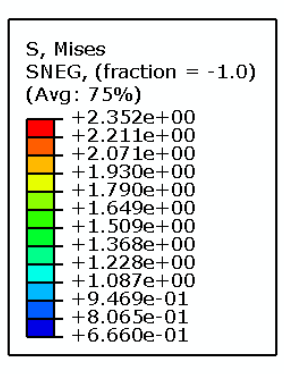

Right Lamboidal Suture

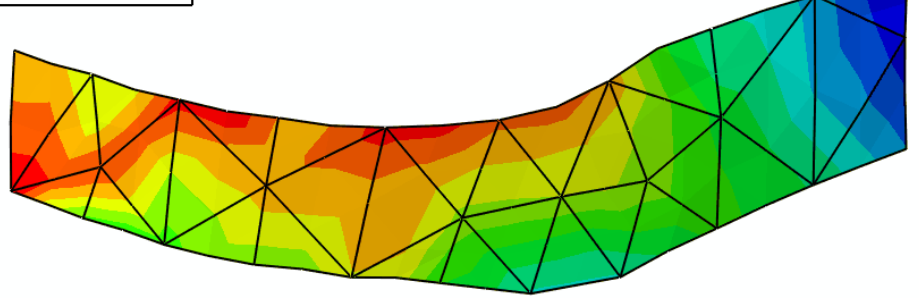

$\mathbf{Z}$ ODB: 1Slug025.odb Abaqus/Standard 6.12-1 Sun Mar 08 22:15:01 Pacific Daylight Time 2015
Xtep: load
$\begin{gathered}\text { Yncrement } \\ \text { Primary Var: S, Step Times }=\end{gathered}$ 
Appendix B: Color Contour Plots of Von Mises Stress Distribution within Suture Segments of Model 2.
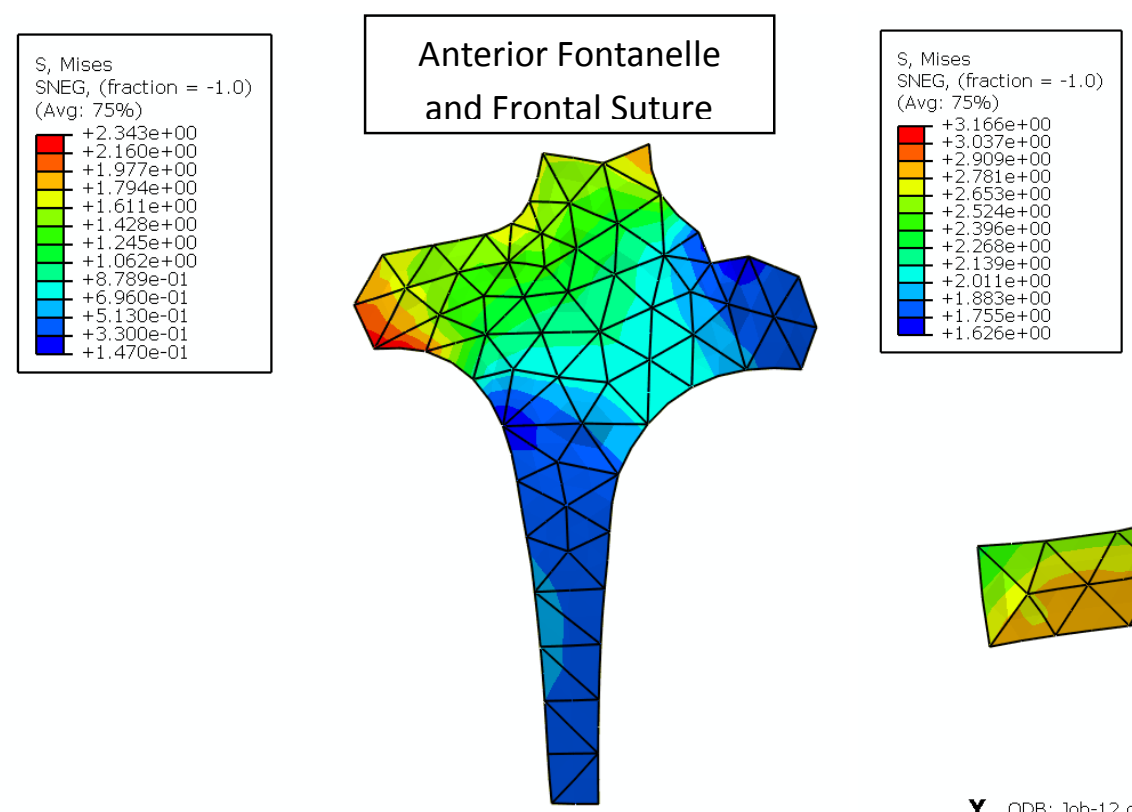

\section{Sagittal Suture}
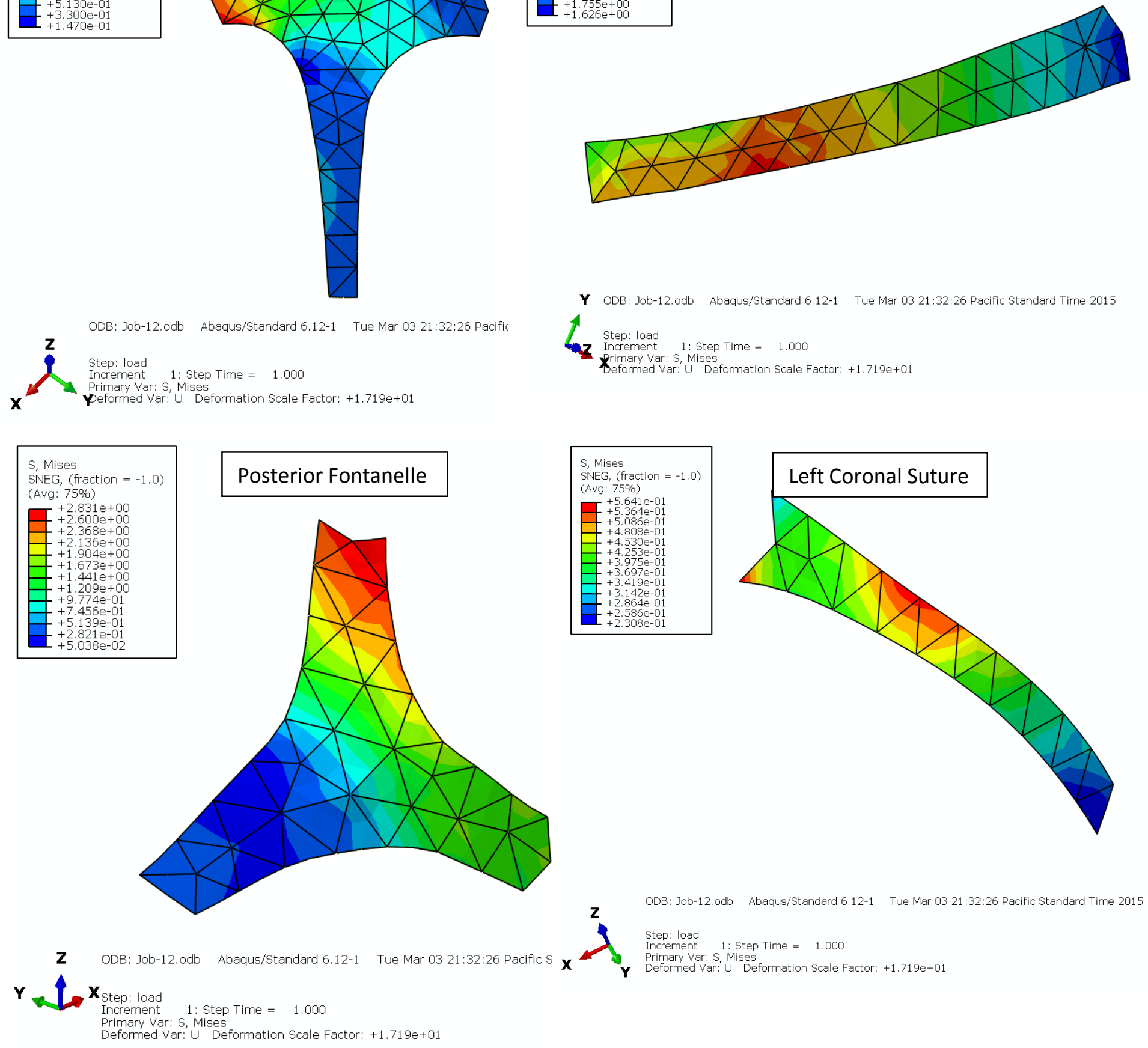

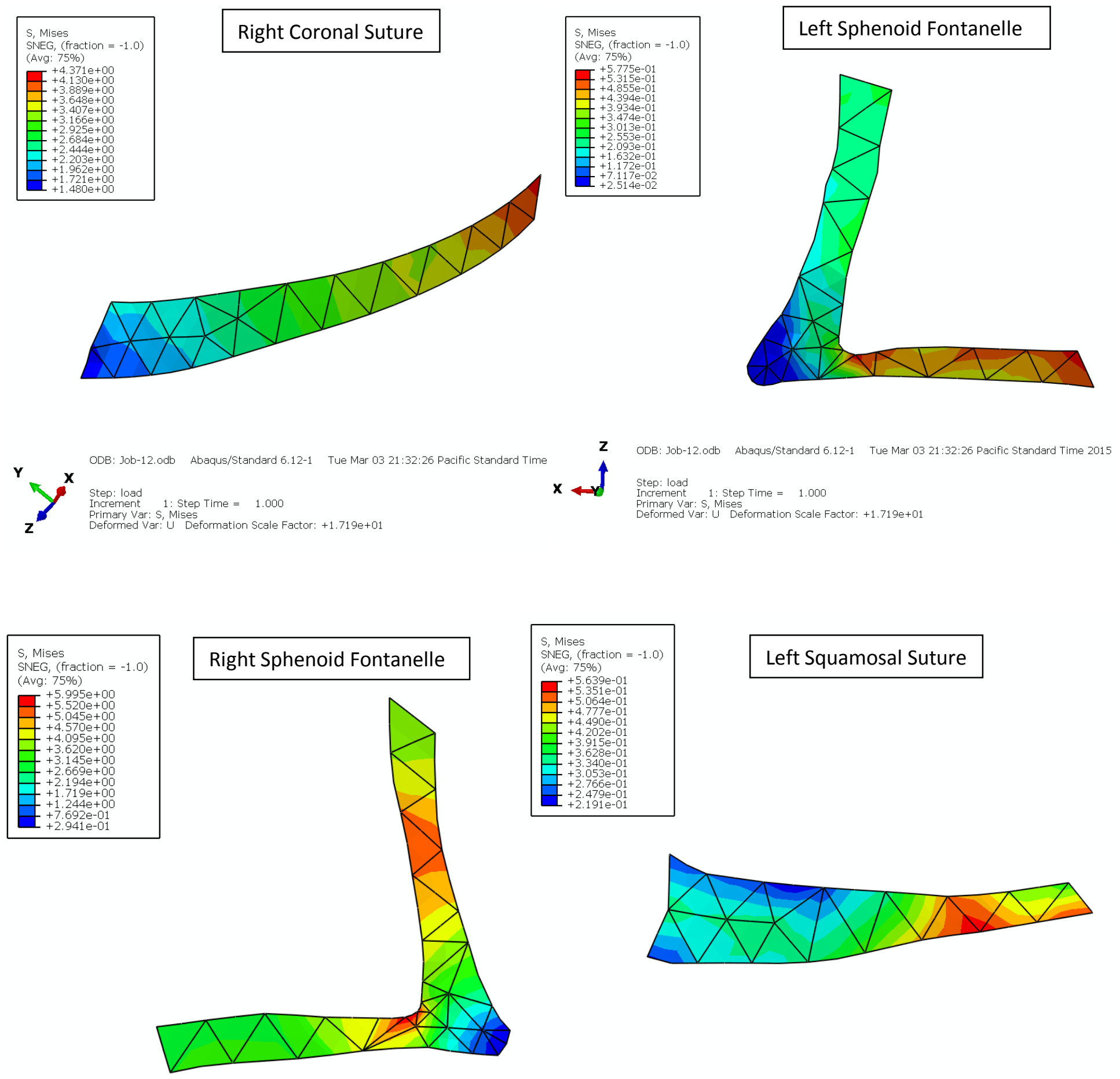

ODB: Job-12.odb Abaqus/Standard 6.12-1 Tue Mar 03 21:32:26 Pacific Standard Time 2015
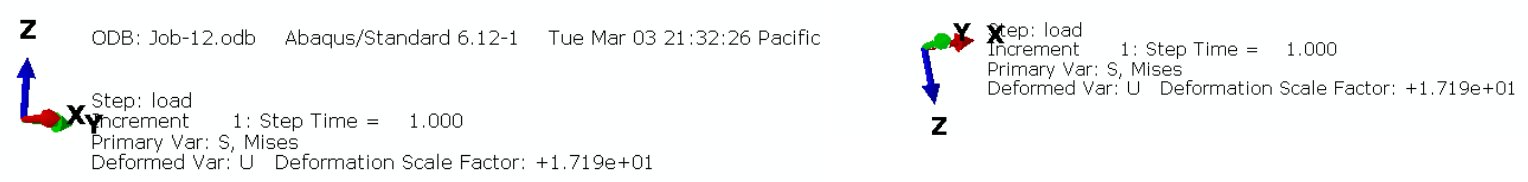

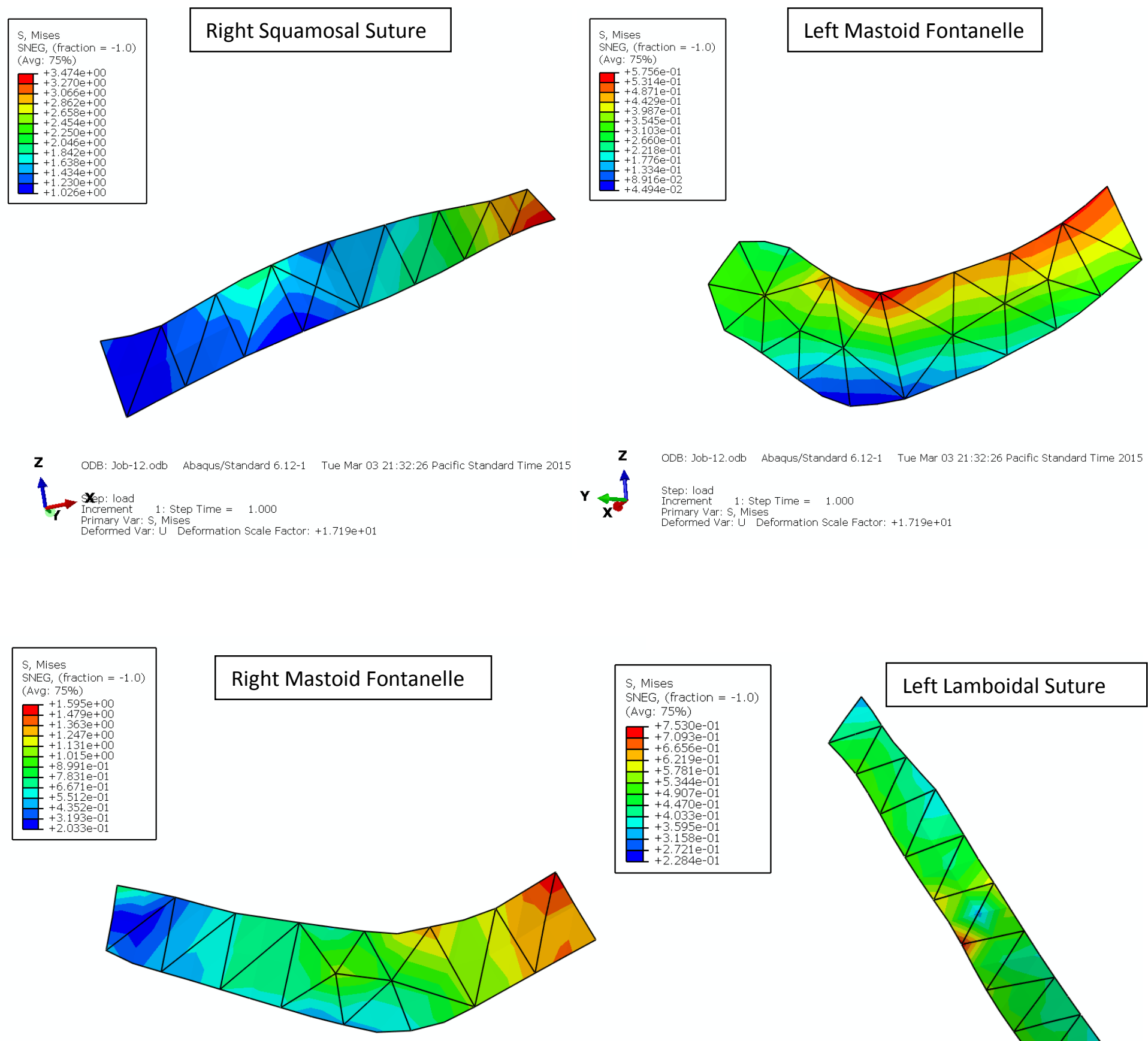

\section{Right Mastoid Fontanelle}

$\mathbf{Z}$

ODB: Job-12.odb Abaqus/Standard 6.12-1 Tue Mar 03 21:32:26 Pacific Standard Time 2015

Ly Xep: load

Increment 1 : Step Time $=1.000$

Primary Var: $S$, Mises
Deformed Var: $U$ Deformation Scale Factor: $+1.719 e+01$

ODB: Job-12.odb Abaqus/Standard 6.12-1 Tue Mar 03 21:32:26 Pacific St

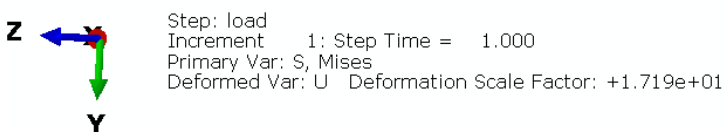




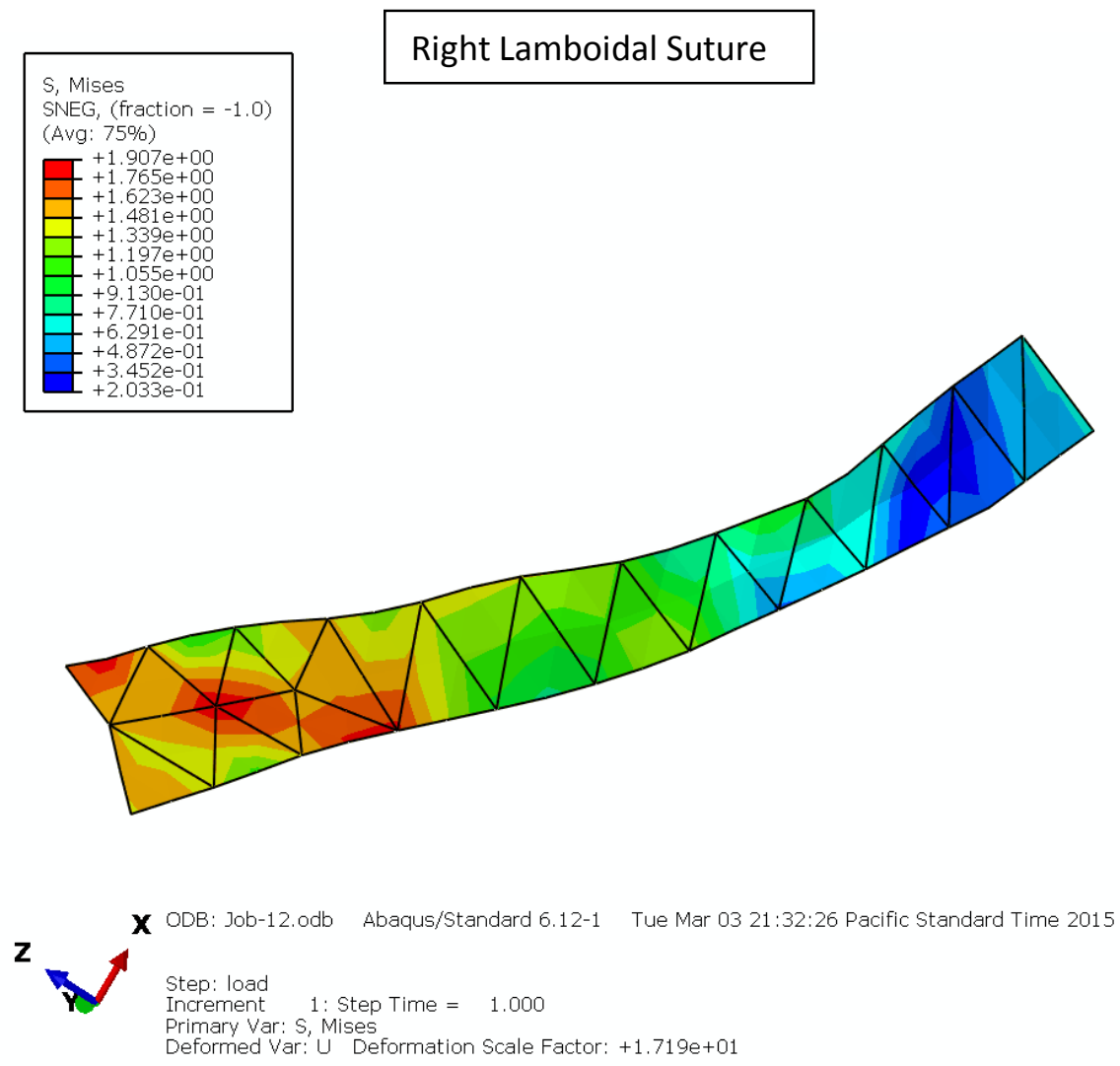


Appendix C: Color Contour Plots of Von Mises Stress Distribution Within Suture Segments of Model 3
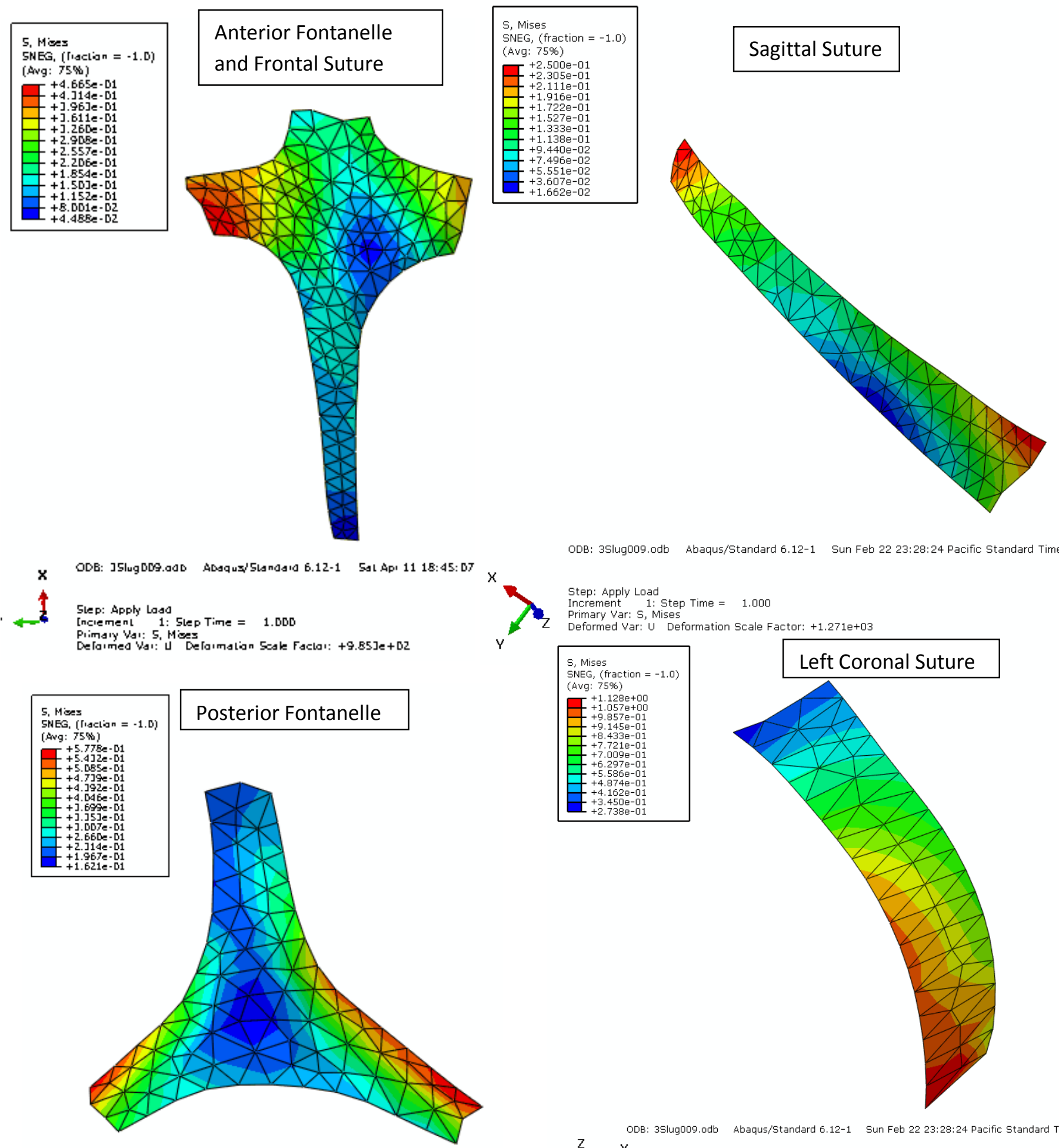


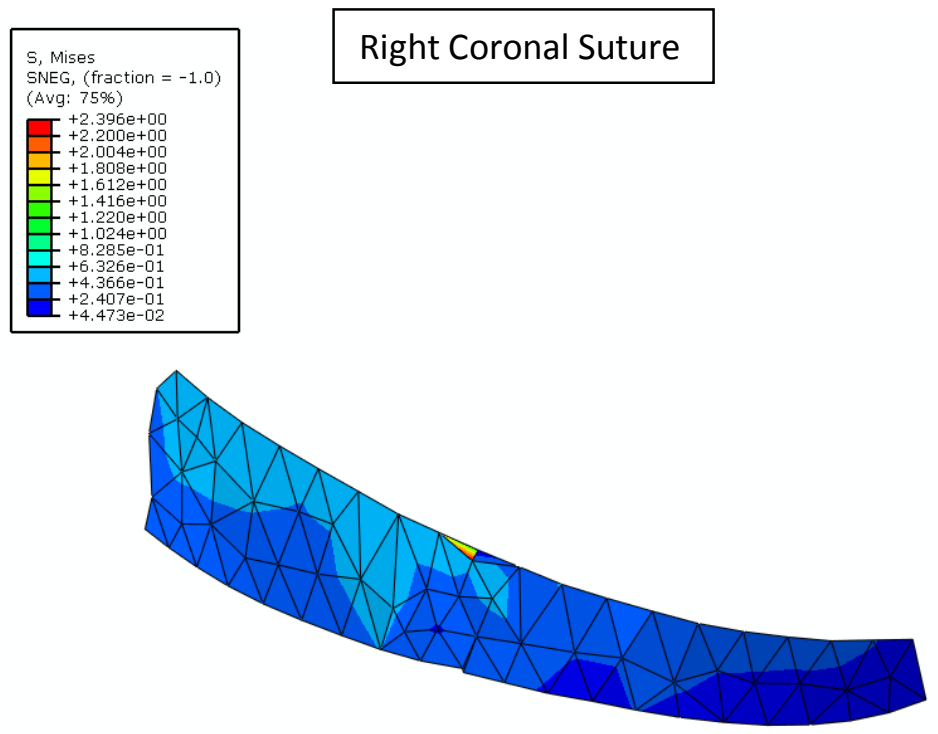

ODB: 3Slug009.odb A.baqus/Standard 6.12-1 Sun Feb 22 23:28:24 Pacific Standard Time 2015

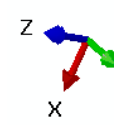

Step: Apply Load
Increment $1:$ Step Time $=1.000$

$Y$ Deformed Var: $U$ Deformation Scale Factor: $+1.271 \mathrm{e}+03$
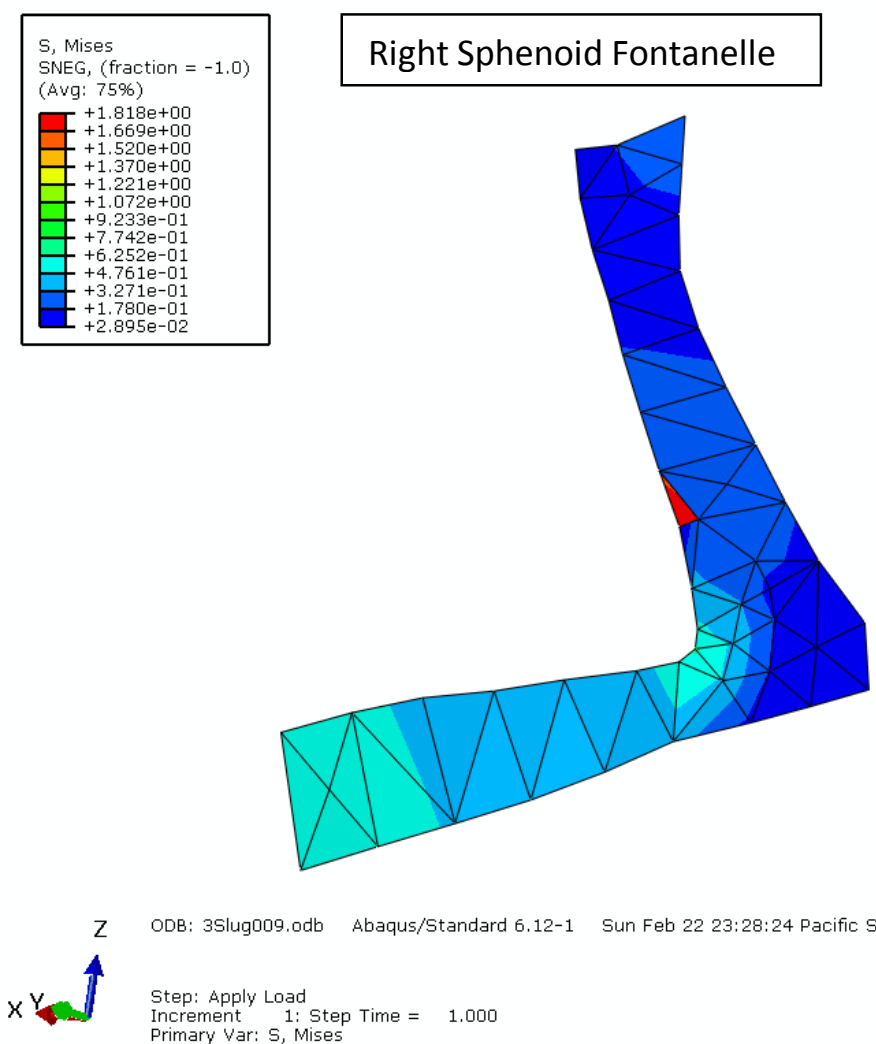

ODB: 3Slug009.odb

Abaqus/Standard 6.12-1

Sun Feb 22 23:28:24 Pacific Standard

Step: Apply Load

Increment 1: Step Time $=1.000$

Deformed Var: U Deformation Scale Factor: +1.271e+03
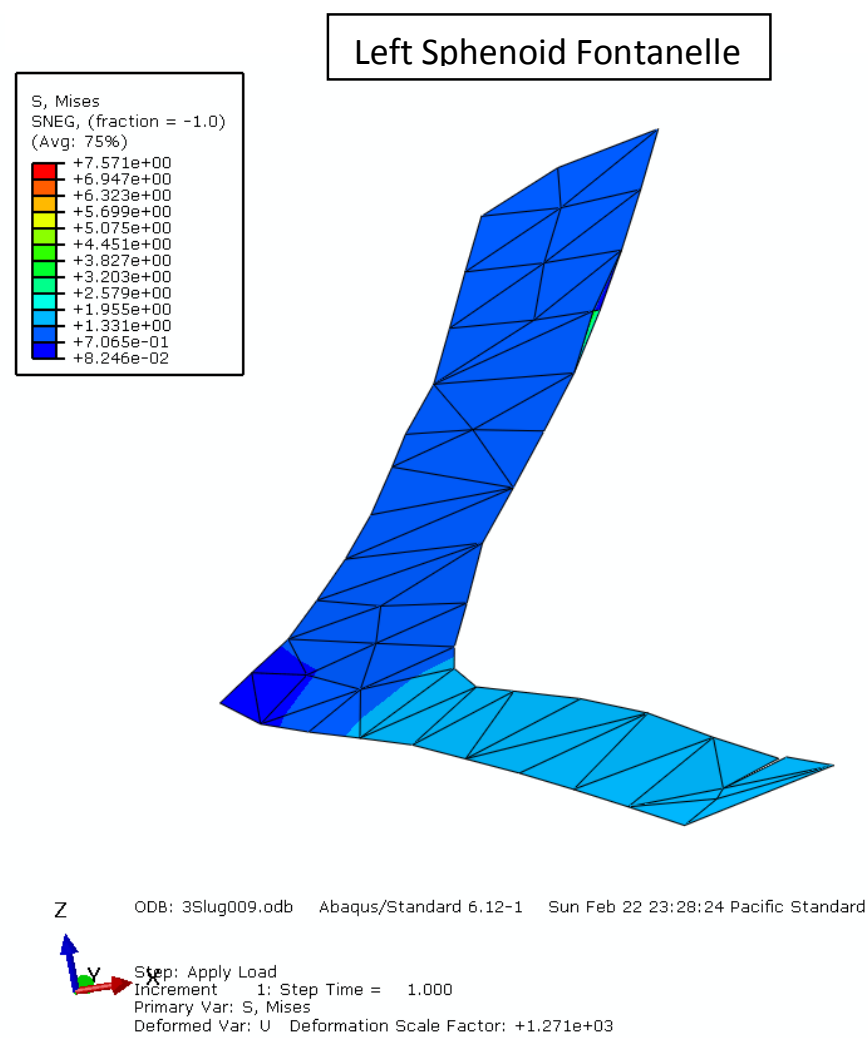

Right Squamosal Suture
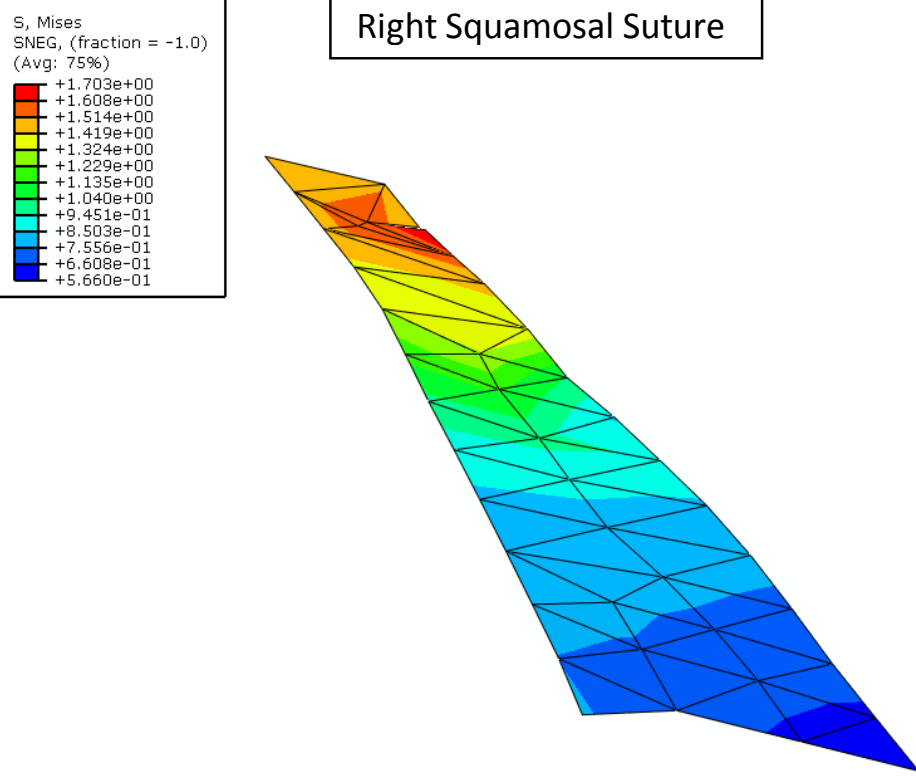

Z ODB: 3Slug009.odb Abaqus/Standard 6.12-1 Sun Feb 22 23:28:24 Pacific Standard Time 2015

Step: Apply Load
Increment 1: Step Time $=1.000$
Primary Var: S, Mises

Xeformed Var: $U$ Deformation Scale Factor: $+1.271 e+03$ 

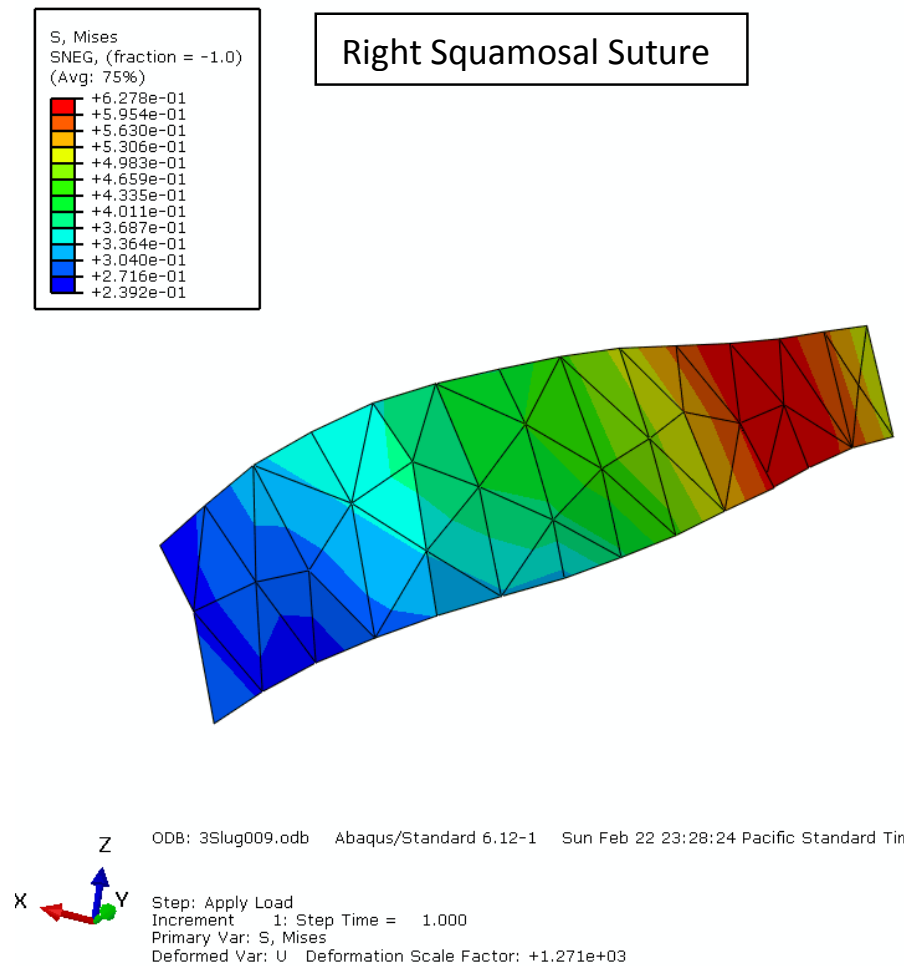

ODB: 3Slug009.odb

Step: Apply Load

Deformed Var: U Deformation Scale Factor: $+1.271 \mathrm{e}+03$

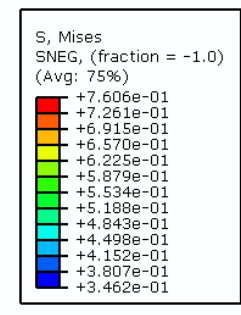

\section{Left Mastoid Fontanelle}
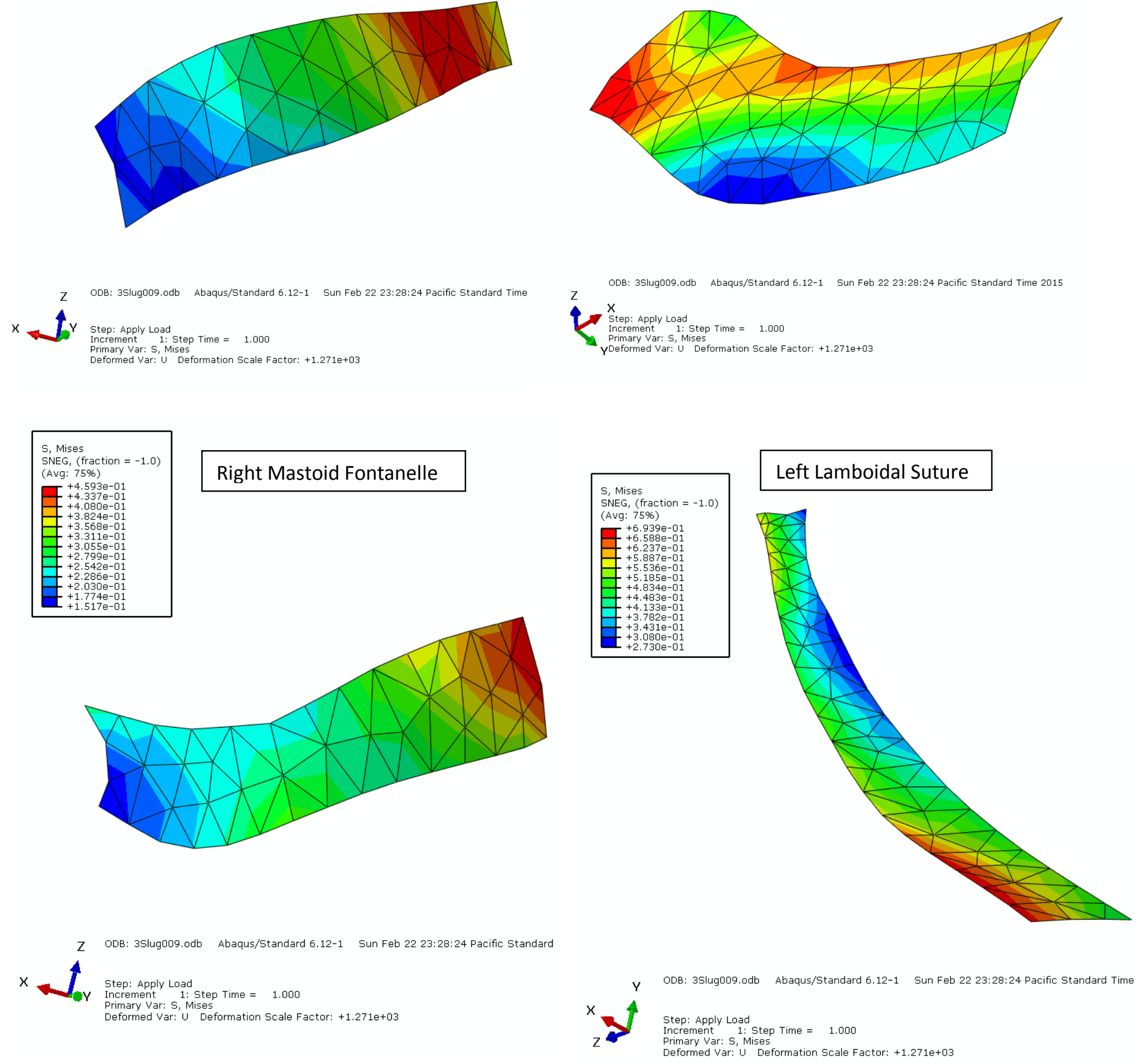


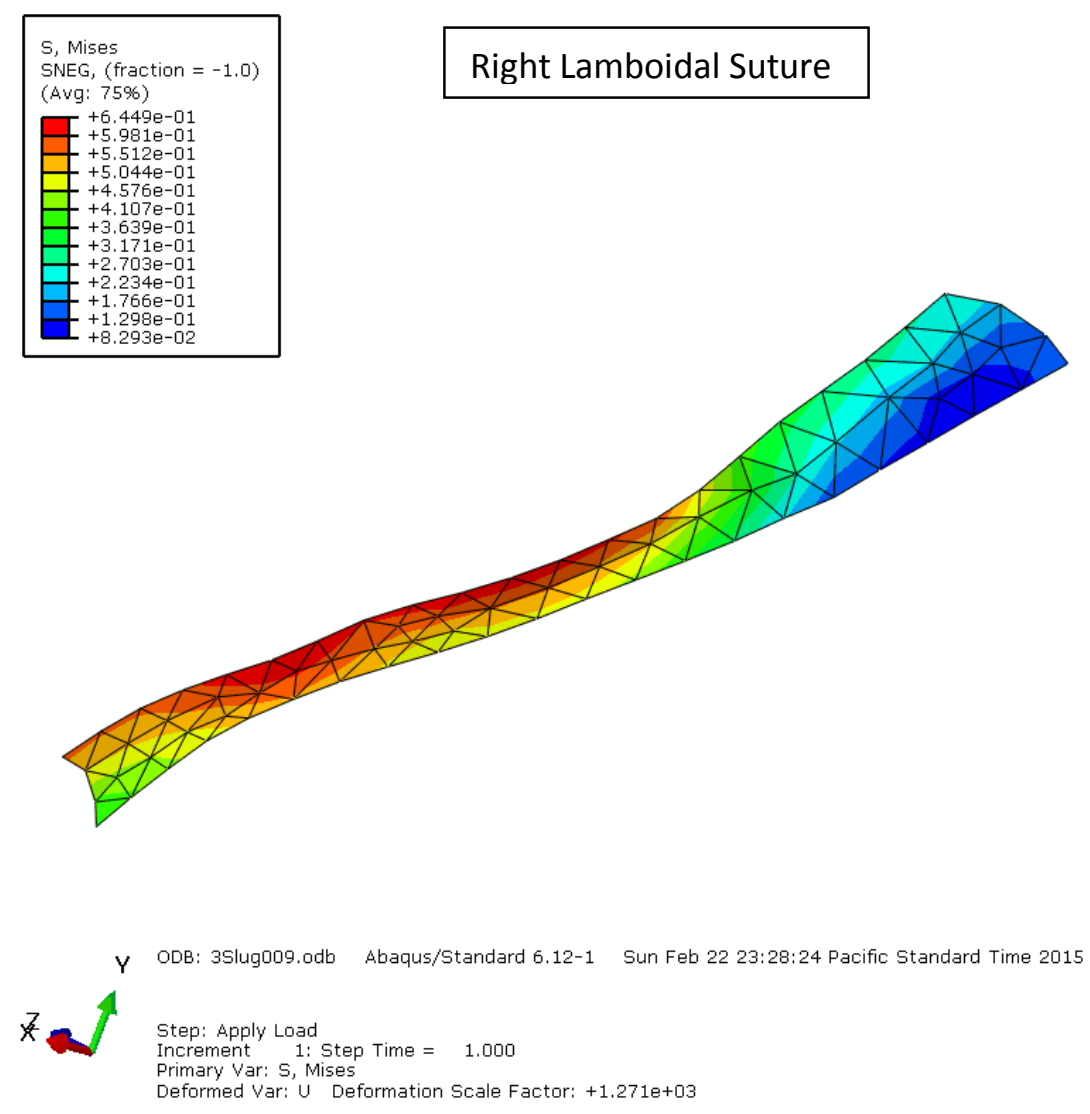

